\title{
Lie Algebras and the Dimension Problem
}

\author{
Dissertation \\ zur Erlangung des mathematisch-naturwissenschaftlichen \\ Doktorgrades \\ "Doctor rerum naturalium"
}

im Promotionsprogramm "School of Mathematical Science" der Georg-August University School of Science (GAUSS)

vorgelegt von

Thomas Sicking

geboren in Steinfurt

Göttingen, 2020 


\section{Betreuungsausschuss}

Erstbetreuer Prof. Dr. Laurent Bartholdi

Mathematisches Institut

Georg-August-Universität Göttingen

and

École Normale Supérieure, Lyon

Zweitbetreuer Prof. Dr. Thomas Schick

Mathematisches Institut

Georg-August-Universität Göttingen

\section{Mitglieder der Prüfungskommission}

Referent Prof. Dr. Laurent Bartholdi

Mathematisches Institut

Georg-August-Universität Göttingen

and

École Normale Supérieure, Lyon

Koreferent Prof. Dr. Thomas Schick

Mathematisches Institut

Georg-August-Universität Göttingen

\section{Weitere Mitglieder der Prüfungskommission}

Prof. Dr. Harald Helfgott

Mathematisches Institut

Georg-August-Universität Göttingen
Prof. Dr. Florin Manea

Institut für Informatik Georg-August-Universität Göttingen

Prof. Dr. Inder Bir S. Passi

Prof. Dr. Roman Mikhailov Centre for Advanced Study in Mathematics, Laboratory of Modern Algebra and Panjab University Chandigarh Applications,St. Petersburg State University and and Indian Institute of Science Education and Research Mohali

Prof. Dr. Stephan Huckemann

Institut für Mathematische Stochastik

Georg-August-Universität Göttingen

Tag der mündlichen Prüfung: 30. November 2020 


\section{Acknowledgements}

First of all, I would like to express my sincere gratitude to my main advisor, Prof. Dr. Laurent Bartholdi, for constantly and patiently supporting me throughout this project by giving constructive input at any time, giving new ideas and new points of view when needed, and supporting me financially and morally whenever necessary.

I would also like to thank my second advisor Prof. Dr. Thomas Schick for giving mathematical and motivational support, particularly towards the later stages of my work.

I am also deeply grateful to Prof. Dr. Inder Bir S. Passi for his warm hospitality during my visit at IISER Mohali in 2015/16. An essential part of my work was created during that visit, and the mathematical and personal experience I gained there will never be forgotten.

I would also like to thank Prof. Dr. Roman Mikhailov, whom I met in India, and whose way of thinking about mathematics is very inspiring.

Thanks also to Prof. Dr. Ulrich Stuhler and Dr. Stefan Wiedmann for the fruitful mathematical discussions during their Oberseminar and the hikes to the Brocken. Many thanks to the secretaries of the mathematical institute for organizing everything behind the scenes for my many contract renewals, and also to the members of the Cafeten-Kollektiv for providing the coffee we are trying to turn into theorems. I wish to thank my parents for their constant support from the day I was born up until now. Their guidance in any circumstances is incredible.

Finally, my biggest thanks goes to my girlfriend Myrtha and our son Levi. Without their constant love, Levi's daily happiness and Myrtha's daily encouragement, this thesis might have never finished. I cannot wish to have better people around me to share my life with. 


\section{Contents}

$\begin{array}{llr}1 & \text { Introduction } & 6\end{array}$

1.1 Groups and Lie Algebras . . . . . . . . . . . . . . . . . . . . . 6

1.2 What are abstract Lie Algebras?. . . . . . . . . . . . . . . . 7

1.3 Dimension and Ore Problems . . . . . . . . . . . . . . . . . . . . 9

1.4 Previous Results . . . . . . . . . . . . . . . . . . . . . . 10

1.5 New Contributions . . . . . . . . . . . . . . . . . . . . . . . 12

$\begin{array}{llr}2 & \text { Preliminaries } & 14\end{array}$

2.1 General Concepts . . . . . . . . . . . . . . . . . . . . . . . . . 14

2.2 Free Lie Algebras . . . . . . . . . . . . . . . . . . . . . . . . . . . . 22

2.3 Hopf Algebras . . . . . . . . . . . . . . . . . . . . . . . . . . . . . . 23

2.4 Simple and Semisimple Lie Algebras . . . . . . . . . . . . . . . . . 27

2.5 Simplicial Objects . . . . . . . . . . . . . . . . . . . . . . . . 31

2.6 The Dimension Subgroup Problem . . . . . . . . . . . . . . . . . . . 34

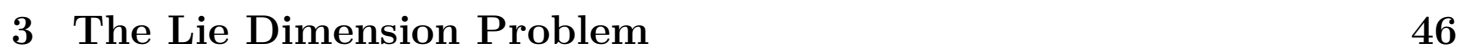

3.1 Some first results . . . . . . . . . . . . . . . . . . . . . . . . . . . . . . . 48

3.2 Sjogren's Theorem for the Lie Dimension Problem . . . . . . . . . . 54

3.3 The Dimension Problem for Metabelian Lie Rings . . . . . . . . . . 62

3.4 Dimension Quotients of Lie rings $\ldots . . . . . .67$

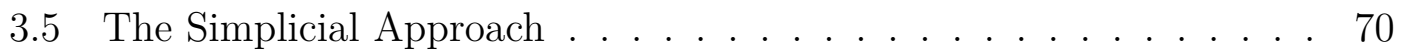

3.6 Comparing the Results . . . . . . . . . . . . . . . . . . . . . . . . 78

\begin{tabular}{|lll}
\hline 4 & The Ore Conjecture for Lie Algebras & 80
\end{tabular}

4.1 Introduction . . . . . . . . . . . . . . . . . . . . . . . . . . . . 80

4.2 Generalized Jacobson-Witt Algebras in one Variable . . . . . . . . . 81

4.3 The case when the field is algebraically closed . . . . . . . . . . . . 83

4.4 The second Jacobson-Witt Algebra . . . . . . . . . . . . . . . . . . 84

4.5 The Higher Jacobson-Witt Algebras . . . . . . . . . . . . . . . . . . 88

4.6 Brown's Strategy $\ldots \ldots \ldots$. . . . . . . . . . . . . . . . . . . . . . 92

4.7 Summarizing the Results . . . . . . . . . . . . . . . . . . . 96

$\begin{array}{lll}5 & \text { Bibliography } & 98\end{array}$ 
There's nothing wrong with occasionally staring out of the window and thinking nonsense, as long as the nonsense is yours.

Lemony Snicket 


\section{Introduction}

\subsection{Groups and Lie Algebras}

Many people have an intuitive understanding of symmetry. For example, a square may seem more symmetric to us than a generic rectangle or a trapezoid. The reason for that is, that the square has four axes of symmetry (namely the diagonals and the bisections of its sides), while the rectangle has only the bisections of its sides, and the trapezoid has non at all in the general case. Also, a rotation by $90^{\circ}$ around the midpoint turns the square into itself, while only a rotation by $180^{\circ}$ does so for the rectangle, and no rotation at all (or only the trivial rotation by $360^{\circ}$ ) for the trapezoid.

Mathematically, one can formalize the concept of symmetry using so-called symmetry groups. The symmetry group of an object is the set of all rotations and reflections that map the object into itself. The symmetry group of a square has then 8 elements, 4 rotations and 4 reflections, while the rectangle has a symmetry group of 4 elements, and the trapezoid a symmetry group of only one element. This justifies the intuitive idea that the square is "more symmetric" than the trapezoid. Now the circle is one of the most symmetric objects one can think of. Namely every single rotation around its center, by $90^{\circ}, 108.65^{\circ}, \sqrt{2}^{\circ}$, or whatever real number you can think of, maps the circle into itself. Also, the reflection by every diameter does so. Thus the symmetry group of the circle is infinite. Also, the group is not discrete, i.e. for any two different rotations by angles $\alpha$ and $\beta$, there is a rotation "in between" those, e.g. the rotation by $(\alpha+\beta) / 2$. This is not the case for the square, where there is no rotation between those by $90^{\circ}$ and $180^{\circ}$, for example.

One may now want to look at transformations "infinitesimally close" to a given transformation to get a better understanding of the symmetry group of the circle. For example, a rotation infinitesimally close to the trivial rotation (i.e. rotation by $0^{\circ}$ ) of the circle maps a point $p$ to an infinitesimal neighborhood of $p$, so one can think of it as being a shift along the line tangent to the circle at $p$. To be slightly more mathematical, the unit circle in the complex plane is given by $S^{1}=\{z \in \mathbb{C}: z \bar{z}=1\}$. Now a rotation of the unit circle is just the multiplication by an element of $S^{1}$, so a map of the type $z \mapsto r z$ with $r \in S^{1}$. The trivial rotation then corresponds multiplication by $r=1$. The tangent line at 1 is a vertical line given by the set $\{1+i t: t \in \mathbb{R}\}$, or in other words the set of translations of 1 on the vertical line. Now suppose $1+\varepsilon i$ on the tangent line is "infinitesimally close" to 1 . Imagine $\varepsilon$ being very small, so $\varepsilon^{2}$ is very very small and can be thought of as zero. Then $(1+\varepsilon i)(\overline{1+\varepsilon i})=1+\varepsilon i-\varepsilon i+\varepsilon^{2} \approx 1$, so $1+\varepsilon i$ is very close to being a rotation. Lie algebras were introduced as exactly that, transformations that are "infinitesimally small" or infinitesimally close to the identity. So we can say now that the Lie algebra of the rotations of the circle is the set of translations along a vertical line. As maps, these are given by addition by an imaginary number, so maps of the type $z \mapsto z+t$ with $t \in i \mathbb{R}$, in other words $t+\bar{t}=0$.

The advantage of Lie algebras is now that they may be easier to analyze. The multiplicative relation $r \bar{r}=1$ of the rotation group becomes an additive relation 
$t+\bar{t}=0$, and addition is somewhat easier than multiplication. This becomes even more clear when looking at symmetry groups in higher dimensions, where the multiplications will be matrix multiplications, and the additions just matrix additions, which are much easier to calculate.

Apart from the geometric intuition, groups can also be defined abstractly, as just a set $G$ with an operation $\circ$, such that the operation is associative (i.e. $(x \circ y) \circ z=x \circ(y \circ z)$ for all $x, y, z \in G)$, has a neutral element $e \in G$ with $e \circ x=x \circ e=x$ for all $x \in G$, and every element $x \in G$ has an inverse $x^{-1} \in G$ with the property that $x \circ x^{-1}=x^{-1} \circ x=e$. One then readily verifies that symmetry groups are indeed groups. But abstract groups can be used in many other branches of mathematics. For example, group theory can be used to show that a generic quintic polynomial equation (i.e. an equation of the form $\left.x^{5}+a x^{4}+b x^{3}+c x^{2}+d x+e=0\right)$ cannot be algebraically solved, or to analyze knots. Now abstract groups do not have a concept of infinitesimality, so one may now wonder if a concept of an abstract Lie algebra exists.

\subsection{What are abstract Lie Algebras?}

To define the notion of an abstract Lie algebra, one may want to see what abstract rules Lie algebras with the geometrical interpretation as above satisfy, and then define an abstract Lie algebra via these rules.

We will recall how the concept of infinitesimality is introduced in high school mathematics. For a real valued differentiable function $f: \mathbb{R} \rightarrow \mathbb{R}$, its differential $f^{\prime}: \mathbb{R} \rightarrow \mathbb{R}$ is another function which describes the behavior of $f$ infinitesimally close to a given point $x \in \mathbb{R}$. One can now wonder how to calculate the differential of a given function. In order to do that, it is helpful to know certain rules, for example how to differentiate the sum of two functions, multiples of a function, or the product of two functions. For example, one easily verifies that for two differentiable functions $f$ and $g$, and a real number $\lambda \in \mathbb{R}$, one has

$$
\begin{aligned}
(f+g)^{\prime} & =f^{\prime}+g^{\prime} \text { and } \\
(\lambda f)^{\prime} & =\lambda f^{\prime} .
\end{aligned}
$$

In other words, the map $f \mapsto f^{\prime}$ is $\mathbb{R}$-linear.

If one wants to calculate the derivative of the product of two differentiable functions $f$ and $g$, there is a little more complicated rule, called the Leibniz rule, given by

$$
(f g)^{\prime}=f^{\prime} g+f g^{\prime} .
$$

Now we have found a set of rules for the derivative, which do not require the initial motivation of the concept of a derivative. Indeed, all we need in the above equations is an addition in (1.1) and (1.3), a multiplication with a scalar from a given ring $k$ in (1.2), and a multiplication of two functions in (1.3), so altogether a $k$-algebra. Then, for a given $k$-algebra $A$, we will call a map $D: A \rightarrow A$ a derivation, if $D$ is $k$-linear and $D(a b)=D(a) b+a D(b)$ for all $a, b \in A$. Note that we do not need to assume $A$ to be commutative, nor even associative, to define 
what a derivation is. Then for example if $k=\mathbb{R}$ and $A=C^{\infty}(\mathbb{R})=\{f: \mathbb{R} \rightarrow \mathbb{R}$ : $f$ is differentiable arbitrary often $\}$, the map $\mathrm{d} / \mathrm{d} x: A \rightarrow A, f \mapsto f^{\prime}$ is a derivation. If $D_{1}$ and $D_{2}$ are derivations of the same algebra $A$, then their composition $D_{1} D_{2}: A \rightarrow A, a \mapsto D_{1}\left(D_{2}(a)\right)$ is not necessarily a derivation, as it may not satisfy the Leibniz rule (for example, $(f g)^{\prime \prime} \neq f^{\prime \prime} g+f g^{\prime \prime}$ in general). However, one can verify that the map $\left[D_{1}, D_{2}\right]=D_{1} D_{2}-D_{2} D_{1}$, called the commutator or Lie bracket of $D_{1}$ and $D_{2}$, is always a derivation (even if $A$ is not commutative, or not associative).

Now the Lie bracket in itself has some properties. Namely, it is bilinear, i.e. $\left[\lambda D_{1}+D_{2}, D_{3}\right]=\lambda\left[D_{1}, D_{3}\right]+\left[D_{2}, D_{3}\right]$ for all $\lambda \in k$ and all derivations $D_{1}, D_{2}$ and $D_{3}$, and similarly in the second argument. It is also anti-symmetric, i.e. $[D, D]=0$ for all derivations $D$, and satisfies the Jacobi identity, that is $\left[\left[D_{1}, D_{2}\right], D_{3}\right]+$ $\left[\left[D_{2}, D_{3}\right], D_{1}\right]+\left[\left[D_{3}, D_{1}\right], D_{2}\right]=0$ for all derivations $D_{1}, D_{2}$ and $D_{3}$.

Then we can abstractly define a Lie algebra as a $k$-module $L$ equipped with a bilinear map $[\cdot, \cdot]: L \times L \rightarrow L$, which is anti-symmetric and satisfies the Jacobi identity. Thus, for all $k$-algebras $A$, the set $\operatorname{Der}(A)$ of all derivations of $A$ is a Lie algebra with the above bilinear map. Also one can check that every associative algebra $A$ becomes a Lie algebra with the operation $[x, y]=x y-y x$ for $x, y \in A$. The example of infinitesimal transformations can be generalized as follows. A Lie group is a group $G$, which is also a smooth manifold, such that the group operation $(g, h) \mapsto g h$ and inversion $g \mapsto g^{-1}$ are both smooth. Basic examples of Lie groups are the group $\mathrm{GL}_{n}(\mathbb{R})$ of invertible $n \times n$ matrices over the real numbers, and its closed subgroups, e.g. the group $\mathrm{SL}_{n}(\mathbb{R})$ of matrices with determinant 1 , the group of matrices of $\mathrm{SL}_{n}(\mathbb{R})$ that satisfy $A A^{T}=1$, denoted by $\mathrm{SO}_{n}(\mathbb{R})$, etc. As smooth manifold, every Lie group has a tangent space at the identity element $T_{e}(G)$, which is a real vector space. Now the group structure of $G$ induces a structure of a Lie algebra on $T_{e}(G)$ via the group commutator $(x, y)=x^{-1} y^{-1} x y$.

For example, if the Lie group $G$ is a subgroup of $\mathrm{GL}_{n}(\mathbb{R})$, the tangent space at the identity matrix is a copy of the set of all $n \times n$-matrices with real entries, $\mathbb{R}^{n \times n}$. As such, it can be shown that it consists of all matrices $A \in \mathbb{R}^{n \times n}$, such that $\exp (t A) \in G$ for all $t \in \mathbb{R}$, where

$$
\exp (A)=\sum_{i=0}^{\infty} 1 / i ! A^{i}
$$

is the matrix exponential. The Lie bracket is given by $[A, B]=A B-B A$. Thus, for example, the Lie algebra of $\mathrm{GL}_{n}(\mathbb{R})$ is the set $\mathbb{R}^{n \times n}$ equipped with the Lie bracket above, denoted by $\mathfrak{g l}_{n}(\mathbb{R})$, and the Lie algebra of $\mathrm{SL}_{n}(\mathbb{R})$, denoted by $\mathfrak{s l}_{n}(\mathbb{R})$, is the subalgebra of $\mathfrak{g l}_{n}(\mathbb{R})$ of all matrices with trace zero.

But also abstract groups and abstract Lie algebras are deeply connected. For a given group $G$, define the group commutator by $(g, h)=g^{-1} h^{-1} g h$. Furthermore, for two subgroups $H_{1}$ and $H_{2}$ of $G$, let $\left(H_{1}, H_{2}\right)$ denote the subgroup of $G$ generated by all commutators $\left(h_{1}, h_{2}\right)$ with $h_{1} \in H_{1}$ and $h_{2} \in H_{2}$. Then the lower central series of $G$ is defined inductively by

$$
\gamma_{1}(G)=G, \gamma_{n+1}(G)=\left(G, \gamma_{n}(G)\right) \text { for } n \geqslant 1
$$


It can be easily shown that $\gamma_{n}(G)$ is a normal subgroup of $G$ for every $n \geqslant 1$, and and that $\gamma_{n}(G) / \gamma_{n+1}(G)$ is central in $G / \gamma_{n+1}(G)$, so in particular these successive quotients are abelian. Therefore, the object

$$
\operatorname{gr}(G):=\bigoplus_{n \geqslant 1} \gamma_{n}(G) / \gamma_{n+1}(G)
$$

is an abelian group, i.e. a $\mathbb{Z}$-module. However, it also carries a (non-trivial) stucture of a Lie algebra by setting

$$
\left[g \gamma_{n+1}(G), h \gamma_{m+1}(G)\right]=(g, h) \gamma_{n+m+1}(G)
$$

for $g \in \gamma_{n}(G)$ and $h \in \gamma_{m}(G)$.

Lie algebras are an interesting subject to study in their own right from an algebraic point of view. The algebraic structure can be defined over any ring, and one can start asking purely algebraic questions about this structure. In this thesis, we will translate some group theoretical questions into the language of Lie algebras and attempt to solve them.

\subsection{Dimension and Ore Problems}

The algebra structure of a Lie algebra offers similarities with the group commutator. Thus, a group theoretic problem involving commutators can be translated into the theory of Lie algebras in the following way.

1. Replace every group commutator by the Lie bracket of the Lie algebra,

2. replace the group operation by addition in the Lie algebra.

For example, the question if every element of a group can be written as a product of at most 2 commutators translates to the question if every element of a Lie algebra can be written as a sum of at most two Lie brackets.

Furthermore, problems about the group ring of a group can be translated into problems about the universal enveloping algebra of a Lie algebra. The augmentation ideal of a group ring $k G$, which is the ideal generated by all $g-1$ for $g \in G$ then translates to the ideal in the universal enveloping algebra generated by the image of $L$ under the map $\iota: L \rightarrow \mathcal{U} L$, which will be called augmentation ideal as well. Thus for example the problem of characterizing the intersection of the powers of the augmentation ideal can be investigated in group rings as well as in Lie algebras.

The original group theoretical problems, whose translations are dealt with in this thesis, are the following.

Problem 1.1 (Dimension Subgroup Problem). Given a group $G$ and a commutative ring $k$, let $\varpi_{k}(G) \subseteq k G$ be the augmentation ideal. For $n \geqslant 1$, characterize the subgroups

$$
\delta_{n, k}(G):=G \cap\left(1+\varpi_{k}^{n}(G)\right)
$$


Problem 1.2 (Ore conjecture). Given a non-abelian finite simple group $G$, can every element of $G$ be written as a commutator?

The translations then are:

Problem 1.3 (Lie Dimension Problem). Given a Lie algebra $L$ over a commutative ring $k$, let $\varpi_{k}(L) \subseteq \mathcal{U} L$ be the augmentation ideal. For $n \geqslant 1$, characterize the subalgebras

$$
\delta_{n, k}(L):=\iota^{-1}\left(\iota(L) \cap \varpi_{k}^{n}(L)\right) .
$$

Problem 1.4. Given a finite dimensional simple Lie algebra $L$, can every element of $L$ be written as a commutator?

In the first part of this thesis, the preliminaries necessary to understand the rest of the thesis will be given. We will start with basic definitions, so even a reader without much knowledge beyond an elementary algebra course shall be able to read the following sections of the thesis. At the end of the preliminaries, an overview of the current state of the art of the Dimension Subgroup Problem will be given, and the techniques used to obtain those results will be explained. In the following part, we try to apply those techniques to the Lie Dimension Problem. It will turn out that some of the known results can be achieved with this. However, for various reasons, there are theorems about Dimension Subgroups whose translation could not be proven, or were even disproven in Lie rings. The last part of the thesis deals with the Ore Conjecture for the generalized Jacobson-Witt algebras, a class of finite dimensional simple Lie algebras over fields of positive characteristic. Here, we were able to prove the Ore Conjecture for some of them.

\subsection{Previous Results}

The Ore Conjecture was established by Oystein Ore in 1951 [34]. In the same paper, he proved it for the alternating groups $A_{n}, n \geqslant 5$. Subsequently, Thompson proved it for the projective special linear groups groups $\operatorname{PSL}_{n}(q)$ (with $(n, q) \neq$ $(2,2),(2,3))$ [49, 50, 51, and for various other classes of finite simple groups in [5, 11, 13] et al. Finally, in 2010 the proof of the Ore conjecture for groups has been completed by Liebeck, O'Brien, Shalev and Tiep [27].

The Ore Conjecture for Lie algebras was first proven for the class of simple Lie algebras $\mathfrak{s l}_{n}(k), n \geqslant 2$, of $n \times n$-matrices with trace zero. The case if $k$ is a field of characteristic zero was handled by Shoda [44], and his result was later extended to arbitrary fields by Albert and Muckenhoupt [1]. Later on, in 1963, G. Brown completed the proof for all classical finite dimensional simple Lie algebras, provided that the cardinality of the ground field $k$ is sufficiently large [6]. In particular, if char $(k)=0$ or $k$ is algebraically closed, and hence infinite, the Ore Conjecture holds for all classical finite dimensional simple Lie algebras.

Contrary to that, there is still no complete answer known to the Dimension Subgroup Problem. While it is easily seen that for every commutative ring $k$, we have $\gamma_{n}(G) \subseteq \delta_{n, k}(G)$, where as above $\gamma_{n}(G)$ denotes the $n$-th term of the lower central series of $G$, a complete characterization of $\delta_{n, k}(G)$ (or the quotient $\delta_{n, k}(G) / \gamma_{n}(G)$ ) is not known. However, if the commutative ring $k$ happens to be a field, a complete 
characterization of the Dimension Subgroups is given in [23] for fields of characteristic zero, and in [26] for fields of positive characteristic. But even for $k=\mathbb{Z}$ (where we omit the index $k$ and just write $\delta_{n}(G)$ ), the dimension subgroups are not fully characterized, and only partial results are known. Therefore, one hopes that for Lie algebras, the problem will be easier accessible, and that the results will give inspiration to solve the original problem.

The characterization $\delta_{n, \mathbb{Q}}(G)=\sqrt{\gamma_{n}(G)}=\left\{g \in G: g^{i} \in \gamma_{n}(G)\right.$ for some $\left.i \geqslant 1\right\}$ in [23] gives of course information about the $n$-th integral dimension subgroups. One can conclude from this that the quotient $\delta_{n}(G) / \gamma_{n}(G)$ is a torsion group for every group $G$. It was actually conjectured that $\delta_{n}(G)=\gamma_{n}(G)$ for every $n \geqslant 1$. While this holds for example for free groups [29] and for groups of prime exponent [9], and also for arbitrary groups if $n \leqslant 3$ [35], E. Rips gave an example of a 2-group $G$ with $\delta_{4}(G) \neq \gamma_{4}(G)$ [39, which was later generalized by N. Gupta, who found a group $G(n)$ for every $n \geqslant 4$ with $\delta_{n}(G(n)) \neq \gamma_{n}(G(n))$ [19].

As mentioned above, the quotient $\delta_{n}(G) / \gamma_{n}(G)$ is a torsion group for every group $G$. We have also seen that for $n \geqslant 4$ this group may not be trivial, and one can start wondering about its group structure, particularly its exponent. This was done later, in 1979, by J.A. Sjogren [45, who gave constants depending only on $n$, but not on the group $G$, bounding the exponent of $\delta_{n}(G) / \gamma_{n}(G)$. The bound he gave is still the best known for general groups.

Theorem 1.5 (Sjogren's Theorem [45, 8]). For every $n \geqslant 1$, there is a constant $c_{n}$ with prime divisors at most $n-2$, such that for every group $G$, we have

$$
\delta_{n}(G)^{c_{n}} \subseteq \gamma_{n}(G)
$$

The constants $c_{n}$ are explicitly given in the cited papers. By the condition on the prime divisors of $c_{n}$, it follows again that $\delta_{n}(G)=\gamma_{n}(G)$ for $n \leqslant 3$. Also, if $G$ is a $p$-group for some prime $p$, it follows that $\delta_{n}(G)=\gamma_{n}(G)$ for all $n \leqslant p+1$.

For $n=4$, the constant provided by Sjogren's Theorem is $c_{4}=2$, which is optimal by the example of Rips mentioned above. For $n=5$, we have $c_{5}=48$. It was shown by Tahara [48] that this can be improved and we actually have $\delta_{5}(G)^{6} \subseteq \gamma_{5}(G)$. Thus one expects that for $n \geqslant 6$, the $c_{n}$ given by Sjogren's Theorem are not optimal, though no improvement on this has been made.

As the computations by Tahara for the fifth dimension quotient are very hard, one may make further assumptions on the group. A lot of work has been done on studying dimension subgroups of metabelian groups, i.e. groups $G$ with $[[G, G],[G, G]]=$ $\{1\}$. The bound on the exponent of the dimension quotient was improved by Gupta [18], though no improvement was made regarding the prime divisors. In the same paper, Gupta showed that for metabelian groups, all dimension quotients are abelian groups. In a subsequent paper, Gupta was able to show that for a metabelian group $G$ the exponent of $\delta_{n}(G) / \gamma_{n}(G)$ is a power of 2 [20]. Also, Gupta, Hales and Passi showed that for every finitely generated metabelian group $G$ there exists $n_{0}(G) \geqslant 1$, such that $\delta_{n}(G)=\gamma_{n}(G)$ for all $n \geqslant n_{0}(G)$ [15].

The techniques to obtain most of the above results are similar. Some results on free groups are known, so one writes the given group $G$ as quotient $G \simeq F / R$ 
of a free group $F$ by a normal subgroup $R \subseteq F$. To get some control over the presentation, one takes a certain kind of presentation, called pre-abelian presentation, and then uses some commutator calculus and certain identities in the group ring $\mathbb{Z} F$ to obtain the results. While this procedure is rather straightforward, the calculations sometimes become very long, as e.g. in Tahara's paper on the fifth dimension subgroup. In [45, to prove his theorem Sjogren applied a more sophisticated method for investigating dimension quotients using spectral sequences arising from filtrations of groups. This approach was further developed by Grünenfelder [14], who constructed two spectral sequences arising from a so-called simplicial resolution of a group. Using this method and the theorems he proves about the spectral sequences, it is possible to reprove that the exponent of $\delta_{4}(G) / \gamma_{4}(G)$ divides 2 for every group $G$. Also one can give certain conditions on $G$, under which $\delta_{4}(G)=\gamma_{4}(G)$ holds. For a detailed description of these methods, see [33, Chapter $5]$.

For Lie algebras, the dimension problem was first attacked by Riley [38]. He was able to prove that if $k$ is a field of arbitrary characteristic, then $\delta_{n, k}(L)=\gamma_{n}(L)$ for every $n \geqslant 1$. It follows again that for $k=\mathbb{Z}$, the dimension quotients $\delta_{n}(L) / \gamma_{n}(L)$ are torsion modules for every $n \geqslant 1$. Bartholdi and Passi started investigating the dimension problem for $k=\mathbb{Z}[3]$. In their work, they get remarkably parallel results to the ones known for groups. Namely, they were able to prove that $\delta_{n}(L)=\gamma_{n}(L)$ if $L$ is a free Lie ring, and also if $n \leqslant 3$. They also gave an example of a Lie ring $L$ with $\delta_{4}(L) \neq \gamma_{4}(L)$. Furthermore, a precise description of the fourth dimension quotient of a finitely generated Lie ring is given. Finally, the simplicial approach is handled, and it turns out that the theorems by Grünenfelder also translate smoothly. A second proof for $\delta_{3}(L)=\gamma_{3}(L)$ is given using this approach.

\subsection{New Contributions}

In the fourth section, some further investigation of the Lie dimension subrings is dealt with. A new proof for the equality of $\gamma_{n}(F)$ and $\delta_{n}(F)$ for free Lie rings $F$ is given. So, as in groups, we will write a Lie algebra $L$ as quotient of a free Lie algebra $L \simeq F / R$. As the universal enveloping algebra of a free Lie algebra on a set $X$ is the free associative algebra $\mathbb{Z}\langle X\rangle$ on the same set, one can view $F$ as the Lie subalgebra of $\mathbb{Z}\langle X\rangle$ generated by $X$.

In Cliff and Hartley's paper [8], where they reprove Sjogren's Theorem in a more elementary way, a lot of theory about free Lie algebras and free associative algebras is used. Therefore it is no big surprise that their approach can be used for the Lie dimension problem as well, giving us eventually exactly the same bound that is known for groups.

Theorem 1.6. For every Lie ring L, we have

$$
c_{n} \delta_{n}(L) \subseteq \gamma_{n}(L)
$$

where the constants $c_{n}$ are exactly those from Theorem 1.5 . 
We will also look more closely into metabelian Lie algebras. Here, it turns out that applying the method of Gupta [18] works much better than in the group case, as some binomial coefficients do not appear. The reason for this is that the Lie bracket is bilinear, i.e. $[a x, y]=a[x, y]$ for all $a \in \mathbb{Z}, x, y \in L$, while the group commutator does not satisfy such an easy equation. Consequently, in a joint work with I.B.S Passi, we were able to significantly improve the bound for groups.

Theorem 1.7. [36] Let $L$ be a metabelian Lie ring. Then, for every $n \geqslant 1$, we have

$$
2 \delta_{n}(L) \subseteq \gamma_{n}(L)
$$

Also, the examples of Gupta given in [19 have a Lie algebra equivalent. Thus, for every $n \geqslant 4$ there is a Lie ring $L(n)$ with $\delta_{n}(L(n)) \neq \gamma_{n}(L(n))$. As these Lie rings are also metabelian, the above bound is the best possible.

The last part of the thesis is about the Ore conjecture for Lie algebras. For groups, as mentioned above, the conjecture was finally proven in 2010 [27]. The proof depends on the classification of finite simple groups, and the conjecture is shown for all classes of finite simple groups separately.

For the classical finite dimensional Lie algebras (over the complex numbers, but also over sufficiently large ground fields of arbitrary characteristic), the Ore conjecture was proven by Brown [6] already in 1963. In contrast to the Ore conjecture for groups, the proof does not depend on the classification of finite dimensional simple Lie algebras over $\mathbb{C}$, but only on the existence and some properties of Cartan subalgebras. Thus the proof is significantly shorter than the one for groups. We will make an attempt to prove the Ore conjecture for a class of simple Lie algebras over field of positive characteristic, namely the generalized Jacobson-Witt algebras $W(m, \underline{n})$, which are roughly speaking derivation algebras of polynomial rings in $m$ variables. Though we were not able to prove the Ore conjecture for all those algebras, we have managed to prove it for the generalized Jacobson-Witt algebras in one variable.

Theorem 1.8. Every element of $W(1, \underline{n})$ can be written as a commutator.

Also, under the assumption that the base field is algebraically closed, we managed to prove the Ore conjecture for the Witt algebra $W(2, \underline{1})$.

Theorem 1.9. Every element of the Witt algebra $W(2, \underline{1})$ over an algebraically closed field can be written as a commutator.

The method used here was unfortunately not easy to generalize to the Witt algebras $W(m, \underline{1})$ with $m>2$. Therefore, we tried a different method to prove the conjecture for those Witt algebras, but did not quite get there. However, we came as close as possible.

Theorem 1.10. Every element of the Witt algebra $W(m, \underline{1})$ over a field of sufficiently large cardinality can be written as sum of at most 2 commutators. 


\section{Preliminaries}

We will start by setting up the basic notations and definitions necessary to understand this thesis. We will assume that the reader knows the most basic definitions occurring in an algebra course, namely basic ring theory and module theory, though not more should be required.

\subsection{General Concepts}

Let $k$ be a commutative ring with a unit.

Definition 2.1. A $k$-module $A$ is called an algebra over $k$, if there is a $k$-bilinear map $: A \times A \rightarrow A$ satisfying

$$
a(x \cdot y)=(a x) \cdot y=x \cdot(a y)
$$

for all $x, y \in A$ and $a \in k$. An algebra is called unital, if there is an element $\mathbf{1} \in A$ (called unit) satisfying $\mathbf{1} \cdot x=x \cdot \mathbf{1}=x$ for all $x \in A$.

Definition 2.2. A $k$-algebra $A$ is called graded, if there is a decomposition

$$
A=\bigoplus_{i \geqslant 0} A_{i}
$$

with $A_{i} \cdot A_{j} \subseteq A_{i+j}$ for all $i, j \geqslant 0$. An element $a \in A_{i}$ for some $i$ will be called homogeneous of degree $i$, and we will sometimes write $\operatorname{deg}(a)=i$.

Definition 2.3. Let $A$ be an algebra over $k$. A $k$-submodule $U$ of $A$ is called a subalgebra of $A$ if $x \cdot y \in U$ for all $x, y \in U$, and an ideal of $A$, if $x \cdot y \in U$ and $y \cdot x \in U$ for all $x \in A$ and $y \in U$.

Definition 2.4. A homomorphism of algebras $A$ and $B$ over $k$ is a $k$-linear map $\phi: A \rightarrow B$ satisfying

$$
\phi(x \cdot y)=\phi(x) \cdot \phi(y)
$$

for all $x, y \in A$. If $A$ and $B$ are algebras with unit $\mathbf{1}_{A}$ and $\mathbf{1}_{B}$ respectively, we will further assume that $\phi\left(\mathbf{1}_{A}\right)=\mathbf{1}_{B}$ for a homomorphism $\phi$.

A homomorphism $\phi: A \rightarrow B$ is called an endomorphism if $A=B$, an isomorphism if $\phi$ is bijective, and an automorphism if $\phi$ is both an endomorphism and an isomorphism.

The set $\operatorname{ker}(\phi):=\{x \in A: \phi(x)=0\}$ is called the kernel of $\phi$, and is always an ideal of $A$.

Proposition 2.5. If $A$ and $B$ are two algebras over the same ring $k$, then the tensor product $A \otimes_{k} B$ is an algebra over $k$ with multiplication $(a \otimes b) \cdot\left(a^{\prime} \otimes b^{\prime}\right)=$ $\left(a \cdot a^{\prime}\right) \otimes\left(b \cdot b^{\prime}\right)$ for $a, a^{\prime} \in A, b, b^{\prime} \in B$ on pure tensors, and extended linearly. 
Definition 2.6. An algebra $A$ will be called associative, if

$$
(x \cdot y) \cdot z=x \cdot(y \cdot z)
$$

holds for all $x, y, z \in A$. In associative algebras, we will write $x y$ instead of $x \cdot y$ for simplicity.

Furthermore, $A$ will be called commutative, if

$$
x \cdot y=y \cdot x
$$

for all $x, y \in A$.

Example 2.7. Let $M$ be a $k$-module. Then the set $\operatorname{End}(M)$ of all $k$-linear maps from $M$ to $M$ becomes an associative $k$-algebra by setting

$$
\begin{aligned}
(a \phi)(x) & =a \phi(x), \\
(\phi+\psi)(x) & =\phi(x)+\psi(x), \text { and } \\
(\phi \cdot \psi)(x) & =\phi(\psi(x))
\end{aligned}
$$

for all $a \in k, x \in M$ and $\phi, \psi \in \operatorname{End}(M)$. The identity map is the unit of $\operatorname{End}(M)$. In particular, if $M$ is a finite dimensional free $k$-module with basis $e_{1}, \ldots, e_{n}$, we can identify $\operatorname{End}(M)$ with $k^{n \times n}$, the set of $n \times n$-matrices over $k$.

Example 2.8. Let $\left\{X_{i}: i \in I\right\}$ be a set of letters indexed by an index set $I$. The polynomial ring $k\left[X_{i}: i \in I\right]$ is defined as the $k$-span of all formal products $\prod_{i \in I} X_{i}^{n_{i}}$, where $n_{i}$ are non-negative integers with only finitely many $n_{i}$ different from 0 . The product of $k\left[X_{i}: i \in I\right]$ is given by

$$
\prod_{i \in I} X_{i}^{n_{i}} \cdot \prod_{i \in I} X_{i}^{m_{i}}=\prod_{i \in I} X_{i}^{n_{i}+m_{i}}
$$

and extended linearly. Then $k\left[X_{i}: i \in I\right]$ becomes a commutative, associative $k$-algebra. It is also graded by $\operatorname{deg}\left(\prod_{i} X_{i}^{n_{i}}\right)=\sum_{i} n_{i}$.

Example 2.9. Let $M$ be a $k$-module. For $i \geqslant 1$, let $T^{\otimes i}(M)=M \otimes \cdots \otimes M$ be the tensor product of $M$ with itself $i$ times, and set $T^{\otimes 0}(M)=k$. The tensor algebra $T(M)$ is then defined by

$$
T(M)=\bigoplus_{i \geqslant 0} T^{\otimes i}(M) .
$$

It becomes a graded associative algebra by defining $\left(a_{1} \otimes \cdots \otimes a_{i}\right) \cdot\left(b_{1} \otimes \cdots \otimes b_{j}\right)=$ $a_{1} \otimes \cdots \otimes a_{i} \otimes b_{1} \otimes \cdots \otimes b_{j}$ for $a_{1} \otimes \cdots \otimes a_{i} \in T^{\otimes i}(M)$ and $b_{1} \otimes \cdots \otimes b_{j} \in T^{\otimes j}(M)$, and extending this linearly.

As $T^{\otimes 1}(M)=M$, we have an embedding $\iota: M \hookrightarrow T(M)$. Then the tensor algebra has the following universal property. For any associative $k$-algebra $A$ and any $k$-linear map $\phi: M \rightarrow A$, there is a unique $\Phi: T(M) \rightarrow A$, such that the diagram

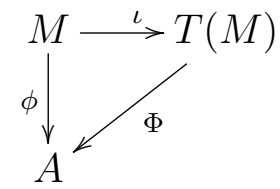

commutes. 
Example 2.10. Let $M$ be a $k$-module and $I$ be the ideal of $T(M)$ generated by all elements of the form $a \otimes b-b \otimes a$ with $a, b \in M$. Then the symmetric algebra $\operatorname{Sym}(M)$ is defined as the quotient $\operatorname{Sym}(M)=T(M) / I$. The embedding $M \hookrightarrow$ $T(M)$ induces an embedding $\iota: M \hookrightarrow \operatorname{Sym}(M)$.

The symmetric algebra also has a universal property. Namely, for any associative, commutative $k$-algebra $A$ and any $k$-linear map $\phi: M \rightarrow A$, there is a unique $\Phi: \operatorname{Sym}(M) \rightarrow A$, such that the diagram

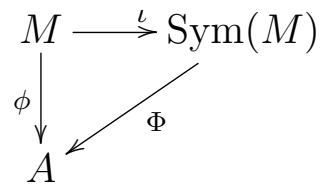

commutes.

Note that if $M$ is a free $k$-module with basis $\left\{e_{i}: i \in I\right\}$, then $\operatorname{Sym}(M) \simeq k\left[X_{i}\right.$ : $i \in I]$.

Definition 2.11. An algebra $L$ over $k$ is called Lie algebra if the bilinear operation - satisfies

$$
\begin{aligned}
x \cdot x & =0, \\
((x \cdot y) \cdot z)+((y \cdot z) \cdot x)+((z \cdot x) \cdot y) & =0
\end{aligned}
$$

for all $x, y, z \in L$. If $k=\mathbb{Z}$, we will call $L$ a Lie ring.

The operation - will then be denoted by $[\cdot, \cdot]$, and will be called Lie commutator, simply commutator or Lie bracket. Equation (2.1) will be referred to as antisymmetry, and equation (2.2) will be called Jacobi identity. Note that the antisymmetry implies that $0=[x+y, x+y]=[x, x]+[x, y]+[y, x]+[y, y]=$ $[x, y]+[y, x]$ for all $x, y \in L$, so $[x, y]=-[y, x]$ for all $x, y \in L$. If the $k$-module $L$ has no 2-torsion, then (2.1) is equivalent to $[x, y]=-[y, x]$ for all $x, y \in L$.

Definition 2.12. A Lie algebra $L$ is called abelian if $[x, y]=0$ for all $x, y \in L$.

The first example of a Lie algebra which one should have in mind is the following. Example 2.13. Let $A$ be an algebra over $k$ (not necessarily associative). A $k$-linear endomorphism $D$ of $A$ is called a derivation if it satisfies the equation

$$
D(x y)=D(x) y+x D(y)
$$

for all $x, y \in A$. Then the set of all derivations is a Lie algebra with the Lie bracket

$$
\left[D_{1}, D_{2}\right]=D_{1} D_{2}-D_{2} D_{1}
$$

We denote by $\operatorname{Der}(A)$ the Lie algebra of derivations of $A$.

Example 2.14. Let $A$ be an associative algebra over $k$. Define

$$
[\cdot, \cdot]: \begin{cases}A \times A & \rightarrow A, \\ (x, y) & \mapsto[x, y]:=x y-y x .\end{cases}
$$

Then $A$ becomes a Lie algebra over $k$ with the above Lie bracket. It is abelian if and only if $A$ is commutative. 
Notation 2.15. If $M$ is a $k$-module, we will denote by $\mathfrak{g l}(M)$ the Lie algebra obtained from $\operatorname{End}(M)$ as in Example 2.14. If $M=k^{n}$, we will also write $\mathfrak{g l}_{n}(k)$ for $\mathfrak{g l}(M)$.

Let $G$ be a group. Define the group commutator as $(g, h)=g^{-1} h^{-1} g h$ for $g, h \in G$. For subgroups $H, K \subseteq G$, denote by $(H, K)$ the subgroup of $G$ generated by all $(h, k)$ with $h \in H, k \in K$. If $H$ and $K$ are normal subgroups, $(H, K)$ is contained in both $H$ and $K$.

The lower central series $\left\{\gamma_{n}(G)\right\}_{n \geqslant 1}$ of $G$ is defined by

$$
\begin{cases}\gamma_{1}(G) & =G, \\ \gamma_{n+1}(G) & =\left(\gamma_{n}(G), G\right) \text { for } n \geqslant 1 .\end{cases}
$$

Then $\gamma_{n}(G)$ is a normal subgroup of $G$ for each $n \geqslant 1$. Also, $\gamma_{n}(G) / \gamma_{n+1}(G)$ is contained in the center of $G / \gamma_{n+1}(G)$ for each $n \geqslant 1$. Therefore, the quotients $\operatorname{gr}_{n}(G)=\gamma_{n}(G) / \gamma_{n+1}(G)$ are abelian groups. Another example of a Lie algebra can be constructed in the following way due to Magnus [30].

Example 2.16. Let $G$ be a group. Then

$$
\operatorname{gr}(G):=\bigoplus_{n \geqslant 1} \operatorname{gr}_{n}(G)
$$

is a graded Lie algebra over $\mathbb{Z}$ with componentwise addition and Lie bracket defined by

$$
\left[g \gamma_{n+1}(G), h \gamma_{m+1}(G)\right]=(g, h) \gamma_{n+m+1}(G) .
$$

for $g \in \gamma_{n}(G), h \in \gamma_{m}(G)$, and extended linearly to $\operatorname{gr}(G)$.

One readily checks that this is indeed a well defined, $\mathbb{Z}$-bilinear operation. In fact, for every group $G$ and every series of subgroups

$$
G=G_{1} \supseteq G_{2} \supseteq G_{3} \ldots
$$

with $\left[G_{n}, G_{m}\right] \subseteq G_{n+m}$ for every $n, m \geqslant 1$, one can define a graded Lie ring structure on $\oplus_{n \geqslant 1} G_{n} / G_{n+1}$ in that way.

Lemma 2.17. Let $A$ be an algebra over $k$, and $\operatorname{Der}(A)$ the Lie algebra of its derivations as in Example 2.13. If $\phi$ is an automorphism of $A$, then

$$
\bar{\phi}: \begin{cases}\operatorname{Der}(A) & \rightarrow \operatorname{Der}(A), \\ D & \mapsto \phi \circ D \circ \phi^{-1}\end{cases}
$$

is an automorphism of $\operatorname{Der}(A)$.

Proof. We first check that $\bar{\phi}(D)$ is a derivation of $A$ whenever $D$ is a derivation of $A$. Indeed, $\bar{\phi}(D)$ is $k$-linear as composition of $k$-linear maps. Also, let $a, b \in A$ be arbitrary. Then

$$
\begin{aligned}
\bar{\phi}(D)(a b) & =\phi\left(D\left(\phi^{-1}(a b)\right)\right)=\phi\left(D\left(\phi^{-1}(a) \phi^{-1}(b)\right)\right) \\
& =\phi\left(D\left(\phi^{-1}(a)\right) \phi^{-1}(b)+\phi^{-1}(a) D\left(\phi^{-1}(b)\right)\right) \\
& =\phi\left(D\left(\phi^{-1}(a)\right)\right) b+a \phi\left(D\left(\phi^{-1}\right)(b)\right)=\bar{\phi}(D)(a) b+a \bar{\phi}(D)(b) .
\end{aligned}
$$


One easily checks that $\bar{\phi} \circ \overline{\phi^{-1}}=\overline{\phi^{-1}} \circ \bar{\phi}=\mathrm{id}$, so $\bar{\phi}$ is invertible with $\overline{\phi^{-1}}$ as its inverse.

For every Lie algebra $L$ one can define the map

$$
\operatorname{ad}: \begin{cases}L & \rightarrow \operatorname{Der}(L), \\ x & \mapsto \operatorname{ad}_{x}\end{cases}
$$

where $\operatorname{ad}_{x}(y)=[x, y]$ for all $y \in L$. By the Jacobi identity, it follows that $\operatorname{ad}_{x}$ is indeed a derivation for every $x \in L$, and $\operatorname{ad}_{[x, y]}=\operatorname{ad}_{x} \operatorname{ad}_{y}-\operatorname{ad}_{y} \operatorname{ad}_{x}$ for all $x, y \in L$.

Definition 2.18. The center $Z(L)$ of a Lie algebra $L$ is the kernel of ad: $L \rightarrow$ $\operatorname{Der}(L)$. In other words, it is the set

$$
Z(L)=\{x \in L:[x, y]=0 \text { for all } y \in L\}
$$

Notation 2.19. For two subsets $X, Y$ of a Lie algebra $L$, we denote by $[X, Y]$ the ideal of $L$ generated by the set $\{[x, y]: x \in X, y \in Y\}$.

Definition 2.20. In analogy to the lower central series of a group, the lower central series $\left\{\gamma_{n}(L)\right\}_{n \geqslant 1}$ of a Lie algebra $L$ is the descending series of ideals of $L$ defined as follows:

$$
\begin{cases}\gamma_{1}(L) & =L \\ \gamma_{n+1}(L) & =\left[\gamma_{n}(L), L\right], n \geqslant 1 .\end{cases}
$$

A Lie algebra is called nilpotent, if $\gamma_{n}(L)=0$ for some $n \geqslant 1$. The nilpotency class of a nilpotent Lie algebra is the maximal $n \geqslant 1$, such that $\gamma_{n}(L) \neq 0$.

By the Jacobi identity and induction on $n$, one verifies that $\left[\gamma_{n}(L), \gamma_{m}(L)\right] \subseteq$ $\gamma_{n+m}(L)$ for all $n, m \geqslant 1$. Then, as in Example 2.16, for a Lie algebra $L$ and $n \geqslant 1$ set $\operatorname{gr}_{n}(L)=\gamma_{n}(L) / \gamma_{n+1}(L)$. Then

$$
\operatorname{gr}(L):=\bigoplus_{n \geqslant 1} \operatorname{gr}_{n}(L)
$$

becomes a graded Lie algebra with Lie bracket $\left[x+\gamma_{n+1}(L), y+\gamma_{m+1}(L)\right]:=$ $[x, y]+\gamma_{n+m+1}(L)$ for $x \in \gamma_{n}(L)$ and $y \in \gamma_{m}(L)$.

Definition 2.21. The derived series $\left\{L^{(n)}\right\}_{n \geqslant 0}$ of a Lie algebra $L$ is the descending series of ideals of $L$ defined as follows:

$$
\begin{cases}L^{(0)} & =L \\ L^{(n+1)} & =\left[L^{(n)}, L^{(n)}\right], n \geqslant 1 .\end{cases}
$$

We will alternatively write $L^{\prime}$ instead of $L^{(1)}, L^{\prime \prime}$ instead of $L^{(2)}$ and so on. A Lie algebra is called solvable, if $L^{(n)}=0$ for some $n \geqslant 0$. For a solvable Lie algebra $L$, the minimal $n \geqslant 0$ such that $L^{(n)}=0$ is called the derived length of $L$. 
By induction on $n$, one easily verifies that $L^{(n)} \subseteq \gamma_{2^{n}}(L)$ for every $n \geqslant 1$. Thus, every nilpotent Lie algebra is solvable. Furthermore, if $n$ is the nilpotency class of $L$, then the derived length of $L$ is at $\operatorname{most} \log _{2} n+1$.

We will now come to the important concept of a universal enveloping algebra. For an associative algebra $A$, denote by $A_{\text {Lie }}$ the Lie algebra obtained from $A$ by Example 2.14.

Definition 2.22. Let $L$ be a Lie algebra. A universal enveloping algebra of $L$ is an associative algebra $\mathcal{U} L$ with unit together with a Lie algebra homomorphism $\iota: L \rightarrow \mathcal{U} L_{\mathrm{Lie}}$, such that for any associative algebra with unit $A$ and any Lie algebra homomorphism $\phi: L \rightarrow A_{\text {Lie }}$, there is a unique homomorphism $\Phi: \mathcal{U} L \rightarrow A$ of associative algebras with unit, such that the diagram

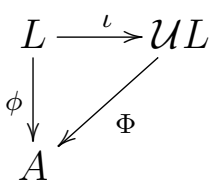

commutes.

Theorem 2.23 ([22, Theorem V.1]). The universal enveloping algebra of a Lie algebra $L$ has the following properties.

1. The universal enveloping algebra is unique up to unique isomorphism.

2. $\mathcal{U} L$ is generated as an associative algebra with unit by $\iota(L)$.

3. Let $L_{1}, L_{2}$ be Lie algebras and $\iota_{i}: L_{i} \rightarrow \mathcal{U} L_{i}, i=1,2$ be their universal enveloping algebras. Let $\phi: L_{1} \rightarrow L_{2}$ be a homomorphism. Then there exists a unique homomorphism $\Phi$ of associative algebras with unit, such that the diagram

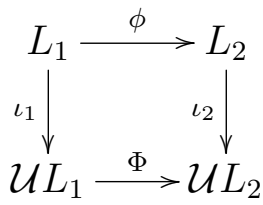

commutes. If $\phi$ is surjective, then $\Phi$ is surjective too.

4. Let $I$ be an ideal of $L$, and let $\mathfrak{I}$ be the ideal of $\mathcal{U} L$ generated by $\iota(I)$. Then $\mathcal{U} L / \mathfrak{I}$ together with the map $\bar{\iota}: x+I \mapsto \iota(x)+\mathfrak{I}$ is a universal enveloping algebra for $L / I$.

Proof. We will give a proof of the second part of the third assertion, which is not given in [22], and an alternate proof of the fourth assertion.

Assume $\phi: L_{1} \rightarrow L_{2}$ is surjective. Then, as $\iota_{2}\left(L_{2}\right)$ generates $\mathcal{U} L_{2}$, and $\iota_{2}\left(L_{2}\right)=$ $\left(\iota_{2} \circ \phi\right)\left(L_{1}\right)=\left(\Phi \circ \iota_{1}\right)\left(L_{1}\right) \subseteq \Phi\left(\mathcal{U} L_{1}\right)$, a generating set of $\mathcal{U} L_{2}$ is contained in the image of $\Phi$, and thus $\Phi$ is surjective.

For a proof of the fourth assertion, let $\iota: L \rightarrow \mathcal{U} L$ and $\iota^{\prime}: L / I \rightarrow \mathcal{U}(L / I)$ be the 
canonical maps. Define $\bar{\iota}: L / I \rightarrow \mathcal{U} L / \mathfrak{I}, x+I \mapsto \iota(x)+\mathfrak{I}$. By the definition of $\mathfrak{I}$, this is a well defined map, therefore, by the universal property, it extends to a homomorphism of associative algebras $\Psi: \mathcal{U}(L / I) \rightarrow \mathcal{U} L / \mathfrak{I}$.

On the other hand, again by the universal property of $\mathcal{U}$, the map $L \rightarrow L / I \rightarrow$ $\mathcal{U}(L / I)$ extends to a homomorphism $\pi: \mathcal{U} L \rightarrow \mathcal{U}(L / I)$. It can easily be seen that $\mathfrak{I} \subseteq \operatorname{ker}(\pi)$, thus this gives a well defined map $\Phi: \mathcal{U} L / \mathfrak{I} \rightarrow \mathcal{U}(L / I)$. We show that $\Psi \circ \Phi$ and $\Phi \circ \Psi$ are the respective identity maps.

As $\mathcal{U} L$ and $\mathcal{U}(L / I)$ are generated by the images of $\iota$ and $\iota^{\prime}$ respectively, it suffices to show the above on elements of the form $\iota(x)+\mathfrak{I}$ and $\iota^{\prime}(x+I)$ with $x \in L$. For those elements, we have

$$
(\Psi \circ \Phi)(\iota(x)+\mathfrak{I})=\Psi\left(\iota^{\prime}(x+I)\right)=\iota(x)+\mathfrak{I},
$$

and

$$
(\Phi \circ \Psi)\left(\iota^{\prime}(x+I)\right)=\Phi(\iota(x)+\mathfrak{I})=\iota^{\prime}(x+I) .
$$

Furthermore, the universal enveloping algebra has the following property.

Lemma 2.24 ([43, Chapter III]). Let $L=L_{1} \times L_{2}$ be a direct product of two Lie algebras. Then

$$
\mathcal{U} L \simeq \mathcal{U} L_{1} \otimes \mathcal{U} L_{2}
$$

If $\iota_{i}: L_{i} \rightarrow \mathcal{U} L_{i}$ denote the canonical homomorphisms, then the homomorphism $\iota: L \rightarrow \mathcal{U} L_{1} \otimes \mathcal{U} L_{2}$ is given by $\iota\left(x_{1}, x_{2}\right)=\iota_{1}\left(x_{1}\right) \otimes 1+1 \otimes \iota_{2}\left(x_{2}\right)$.

So far, we only have only listed properties of the universal enveloping algebra without constructing it explicitly. This can be done in the following way.

Theorem 2.25 ([43, Chapter III]). Let $T(L)$ be the tensor algebra of the $k$-module $L$ as defined in Example 2.9, and let $I \subseteq T(L)$ be the ideal generated by all elements of the form $x \otimes y-y \otimes x-[x, y]$ with $x, y \in L$. Then a universal enveloping algebra can be constructed by

$$
\mathcal{U} L:=T(L) / I,
$$

and $\iota: L \rightarrow \mathcal{U} L$ is given by $x \mapsto x+I$.

Remark 2.26. From the construction, one sees that for an abelian Lie algebra $L$ we have $\mathcal{U} L \simeq \operatorname{Sym}(L)$ as associative algebras. This is also obvious from the universal property, as the image of an abelian Lie algebra under a homomorphism $L \rightarrow A_{\text {Lie }}$ is a commutative subalgebra of $A$. By the universal property of the symmetric algebra, this factors through $\operatorname{Sym}(L)$.

One would normally assume that the map $\iota: L \rightarrow \mathcal{U} L$ is injective. However, this need not be the case, as the following example due to Cartier [7] shows.

Example 2.27. Let $k=\mathbb{F}_{2}\left[X_{0}, X_{1}, X_{2}\right] /\left(X_{0}^{2}, X_{1}^{2}, X_{2}^{2}\right)$, and let $L$ be the Lie algebra given by the presentation

$$
L=\left\langle e_{0}, e_{1}, e_{2} \mid \sum_{i=0}^{2} X_{i} e_{i}=0\right\rangle .
$$

Then $0 \neq x:=\sum_{i<j} X_{i} X_{j}\left[e_{i}, e_{j}\right]$, but $\iota(x)=0$. 
Proof. We first show that $x \neq 0$. Let $F$ be the free Lie algebra over $k$ on the generators $e_{0}, e_{1}, e_{2}$, and let $R$ be the ideal of $F$ generated by $\sum_{i} X_{i} e_{i}$, so that $L \simeq F / R$. Then $R$ is a homogeneous ideal, generated by one element of degree 1 , and $x$ is homogeneous of degree 2. Suppose $x \in R$. Then there are $f_{i} \in k$, such that

$$
x=\left[\sum_{i=0}^{2} X_{i} e_{i}, \sum_{i=0}^{2} f_{i} e_{i}\right]=\sum_{i<j}\left(X_{i} f_{j}-X_{j} f_{i}\right)\left[e_{i}, e_{j}\right] .
$$

This (using $\operatorname{char}(k)=2$ ) leads to the following three equations in $k$.

$$
\begin{aligned}
& X_{0} f_{1}+X_{1} f_{0}=X_{0} X_{1}, \\
& X_{0} f_{2}+X_{2} f_{0}=X_{0} X_{2}, \\
& X_{1} f_{2}+X_{2} f_{1}=X_{1} X_{2} .
\end{aligned}
$$

Now, $k$ is an 8-dimensional $\mathbb{F}_{2}$-vector space with basis $\left\{1, X_{0}, X_{1}, X_{2}, X_{0} X_{1}, X_{0} X_{2}\right.$, $\left.X_{1} X_{2}, X_{0} X_{1} X_{2}\right\}$, so every $f_{i}$ is a sum of those basis elements. By equation (2.5), either $f_{1}$ is divisible by $X_{1}$ or $f_{0}$ is divisible $X_{0}$, but not both. By symmetry, we can assume without loss of generality that $f_{1}$ is divisible by $X_{1}$. Then $f_{0}$ is not divisible by $X_{0}$. Therefore, by equation (2.6), $X_{2}$ divides $f_{2}$. But then the coefficient of $X_{1} X_{2}$ on the left hand side of equation (2.7) is $1+1=0$, leading to a contradiction. Thus $x \neq 0$.

Let now $A$ be an arbitrary associative algebra over $k$, and let $\phi: L \rightarrow A_{\text {Lie }}$ be a homomorphism. Then

$$
\begin{aligned}
\phi(x) & =\sum_{i<j} X_{i} X_{j}\left(\phi\left(e_{i}\right) \phi\left(e_{j}\right)-\phi\left(e_{j}\right) \phi\left(e_{i}\right)\right) \\
& =\left(X_{0} \phi\left(e_{0}\right)+X_{1} \phi\left(e_{1}\right)+X_{2} \phi\left(e_{2}\right)\right)^{2}=\phi\left(\sum_{i} X_{i} e_{i}\right)^{2}=\phi(0)=0 .
\end{aligned}
$$

Therefore, $x$ maps to zero under every homomorphism into an associative algebra, so in particular $\iota(x)=0$.

By the above example, $\iota$ need not be a monomorphism, but in the following cases it is:

1. $L$ is a free $k$-module [52],

2. $k$ is a principal ideal domain [7],

3. $k$ is an algebra over the rationals [10].

So in particular, when $k=\mathbb{Z}$ or $k$ is a field, either of which will always be the case in this thesis, $\iota$ is a monomorphism.

Proposition 2.28. Let $L$ be a Lie algebra over a commutative ring $k$. Then

$$
\mathcal{U} L \simeq k \text { if and only if } L=0 \text {. }
$$


Proof. Suppose first $L=0$. Then $T(L) \simeq k$ and the ideal $I$ as above is the zero ideal, so $\mathcal{U} L \simeq k$.

For the converse, suppose that $\mathcal{U} L \simeq k$. Let $\operatorname{End}(L)$ be the algebra of $k$-module endomorphisms of $L$, and ad: $L \rightarrow \operatorname{End}(L)$ the adjoint representation. Then, by the universal property, there is a unique homomorphism $\Phi: k \rightarrow \operatorname{End}(L)$ extending ad. This must be the map $a \mapsto a \mathrm{id}_{L}$.

Now by anti-symmetry, it follows that $\iota: L \rightarrow k$ is the zero map, and therefore ad is the zero map, thus $L$ is abelian. It follows that $I$ is the ideal of $T(L)$ generated by all $x \otimes y-y \otimes x$ with $x, y \in L$, and $t \in I$ for all $t \in L$. This is only possible if $L=0$.

By the above Proposition and the universal property of $\mathcal{U} L$, the map $L \rightarrow 0$ extends to a map $\varepsilon: \mathcal{U} L \rightarrow k$. This map will be called the augmentation map. We call $\varpi(L)=\operatorname{ker}(\varepsilon)$ the augmentation ideal of $\mathcal{U} L$. It is the two-sided ideal of $\mathcal{U} L$ generated by the image of $L$ under $\iota$. As $k$-modules, we have the decomposition $\mathcal{U} L=k \oplus \varpi(L)$.

If $L$ is a free $k$-module, there is an even stronger theorem constructing a basis of $\mathcal{U} L$ from a given basis of $L$ (see e.g. [22, Theorem V.3] for a reference).

Theorem 2.29 (Poincaré-Birkhoff-Witt). Let L be a Lie algebra over $k$, which is free as $k$-module with basis $\mathcal{B}=\left\{b_{i}\right\}_{i \in I}$. Assume that $I$ is totally ordered by some ordering $\leqslant$. Then the set

$$
\operatorname{PBW}(\mathcal{B})=\left\{b_{i_{1}} \cdots b_{i_{n}}: n \geqslant 0, i_{1} \leqslant i_{2} \leqslant \ldots \leqslant i_{n}\right\}
$$

is a basis of $\mathcal{U L}$ (where we identify $b_{i_{j}}$ with $\iota\left(b_{i_{j}}\right)$ in $\left.\mathcal{U} L\right)$. In particular, we have $\mathcal{U} L \simeq \operatorname{Sym}(L)$ as $k$-modules.

\subsection{Free Lie Algebras}

In the third section of this thesis, the concept of a free Lie algebra will play an important role. Therefore, we will give some basic facts about free Lie algebras here.

Definition 2.30. Let $X$ be a set, $F$ a Lie algebra over $k$, and $\iota: X \rightarrow F$ a mapping. The Lie algebra $F$ is called free on $X$, if for every Lie algebra $L$ over $k$ and every mapping $\phi: X \rightarrow L$, there is a unique Lie algebra homomorphism $\Phi: F \rightarrow L$ extending $\phi$ (i.e. $\phi=\Phi \circ \iota)$.

For every set $X$, there exists a free Lie algebra on $X$, and it is unique up to isomorphism (see [37, Theorem 0.4]). In the same way, for every set $X$ there exists a free associative $k$-algebra on $X$ ([37, p. 6]), denoted by $k\langle X\rangle$, which is unique up to isomorphism. It can be described as the set of all $k$-linear combinations of (finite) words in $X$. By Example 2.14, we can view $k\langle X\rangle$ as a Lie algebra. Given an arbitrary Lie (associative) algebra, it can be written as quotient of a free Lie (associative) algebra on a set of generators of the given Lie (associative) algebra. 
Such a quotient is called a presentation. For a subset $\mathcal{R}$ of a free Lie algebra $F$ on $X$, we will write

$$
L=\langle X \mid \mathcal{R}\rangle
$$

if $L \simeq F / R$, where $R$ is the ideal of $F$ generated by $\mathcal{R}$. As every homomorphism $F \rightarrow A_{\text {Lie }}$, where $F$ is free on $X$, is just a map $X \rightarrow A$, which extends to a homomorphism of associative algebras $k\langle X\rangle \rightarrow A$, we get a connection between free Lie algebras and free associative algebras.

Theorem 2.31 ([37, Theorem 0.5]). Let $F$ be the free Lie algebra on a set $X$. Then $\mathcal{U} F$ is the free associative algebra on the set $X$. Furthermore, $F$ is isomorphic to the Lie subalgebra of $k\langle X\rangle_{\text {Lie }}$ generated by $X$.

Thus, by Theorem 2.23, if a Lie algebra $L$ is given by a presentation $L \simeq F / R$ with a free Lie algebra $F$, the projection $F \rightarrow L$ leads to a surjective homomorphism $\mathcal{U} F \rightarrow \mathcal{U} L$, whose kernel is the ideal $\mathfrak{r}$ of $\mathcal{U} F$ generated by the image of $R$ under the map $F \rightarrow \mathcal{U} F$. Therefore, a presentation of a Lie algebra gives a presentation of its universal enveloping algebra.

Every free Lie algebra has a natural grading. Namely, if $F$ is a free Lie algebra on a set $X$, then the $n$-th homogeneous component $\mathfrak{F}_{n}$ of $F$ is the $k$-module generated by all commutators of $n$ elements of $X$. By the Jacobi identity, the left-normed commutators $\left[X_{i_{1}} \ldots, X_{i_{n}}\right]$ suffice to generate $\mathfrak{F}_{n}$.

\subsection{Hopf Algebras}

One can define also the notion of an associative algebra with a unit in another way.

Definition 2.32. A $k$-module $A$ is called an associative algebra with unit if there exist $k$-linear maps $m: A \otimes A \rightarrow A$, and $\eta: k \rightarrow A$ called multiplication and unit, such that the diagrams

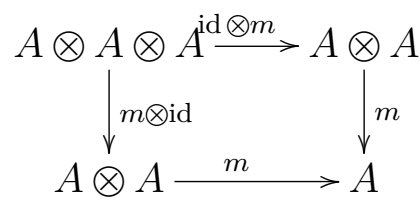

and

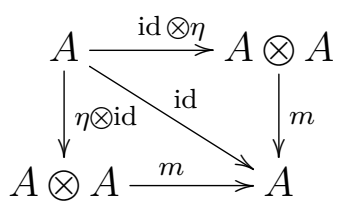

commute. Here, for the definitions of the maps $\eta \otimes$ id and id $\otimes \eta$, we identify $A$ with $k \otimes A$ and $A \otimes k$ respectively.

This notion of an associative algebra allows us to define a coalgebra by reversing the arrows. 
Definition 2.33. A $k$-module $C$ is called a coalgebra, if there exist $k$-linear maps $\Delta: C \rightarrow C \otimes C$ and $\varepsilon: C \rightarrow k$, called comultiplication and counit, such that the diagrams

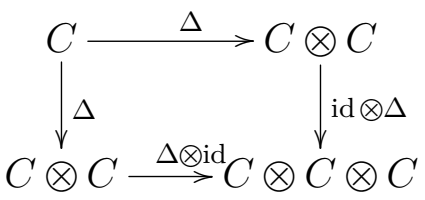

and

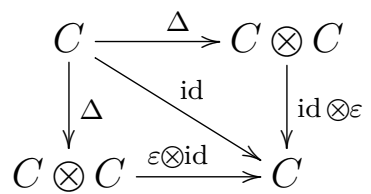

commute. Here, as before, for the definition of the maps $\varepsilon \otimes$ id and id $\otimes \varepsilon$, we identify $C$ with $k \otimes C$ and $C \otimes k$ respectively.

Example 2.34. Let $C$ be a free $k$-module with basis $\mathcal{B}$. Then we can define a coalgebra structure on $C$ by setting

$$
\Delta(b)=b \otimes b
$$

and

$$
\varepsilon(b)=1
$$

for all $b \in \mathcal{B}$, and extending the maps linearly.

An ideal of an associative algebra can be defined as follows.

Definition 2.35. A linear subspace $I$ of an associative algebra $A$ is called an ideal if $m(A \otimes I+I \otimes A) \subseteq I$.

It is well known that the quotient space $A / I$ becomes an algebra in the natural way, by defining $\bar{m}:(A / I) \otimes(A / I) \rightarrow A / I,(a+I) \otimes(b+I) \mapsto m(a \otimes b)+I$, and $\bar{\eta}=\pi \circ \eta: k \rightarrow A / I$, where $\pi$ denotes the projection $A \rightarrow A / I$. Reversing arrows then gives the definition of a co-ideal.

Definition 2.36. A linear subspace $I$ of a coalgebra $C$ is called a co-ideal if $\Delta(I) \subseteq C \otimes I+I \otimes C$ and $I \subseteq \operatorname{ker}(\varepsilon)$

The second condition is necessary to get a well-defined natural counit on the quotient $C / I$ by defining $\bar{\varepsilon}(c+I)=\varepsilon(c)$ for all $c \in C$. The quotient space $C / I$ becomes then a coalgebra in the natural way.

Definition 2.37. A $k$-module $A$ is called a bialgebra if $A$ is an algebra and a coalgebra, such that the comultiplication $\Delta$ and the counit $\varepsilon$ are homomorphisms of associative algebras. Equivalently, $A$ is a bialgebra, if the multiplication $m$ and the unit $\eta$ are homomorphisms of coalgebras. A linear subspace $I \subseteq A$ is called a $b i$-ideal, if it is an ideal and a co-ideal at the same time. 
Let $A$ be an associative algebra and $C$ be a coalgebra with $m, \eta, \Delta, \varepsilon$ the multiplication, unit, comultiplication and counit. Denote by $\operatorname{Hom}(C, A)$ the set of module homomorphisms from $C$ to $A$. Then one can verify that $\operatorname{Hom}(C, A)$ becomes a $k$-algebra with multiplication given by $f * g=m \circ(f \otimes g) \circ \Delta$, which we will call convolution product. The unit element is $\eta \varepsilon$.

Example 2.38. Take $A=k$, then $\operatorname{Hom}(C, A)=C^{*}$, the dual space. Therefore, the dual space of any coalgebra is an associative algebra.

Definition 2.39. Let $H$ over $k$ be a bialgebra, and denote by $H^{a}$ and $H^{c}$ the underlying algebra and coalgebra respectively. If id $\in \operatorname{Hom}\left(H^{c}, H^{a}\right)$ is invertible with respect to $*$, then we will call $H$ a Hopf algebra. The unique inverse of id will be called antipode, and will be denoted by $s$.

Definition 2.40. A Hopf ideal $I$ of a Hopf algebra $H$ is a bi-ideal of the bialgebra $H$ such that $s(I) \subseteq I$.

If $I$ is a Hopf ideal of $H$, then the quotient becomes a Hopf algebra with antipode $\bar{s}: H / I \rightarrow H / I, h+I \mapsto s(h)+I$.

For finite dimensional Hopf algebras $H$, there is a canonical isomorphism $\phi: H^{*} \otimes$ $H^{*} \rightarrow(H \otimes H)^{*}$, with $\phi(f \otimes g)\left(h_{1} \otimes h_{2}\right)=f\left(h_{1}\right) g\left(h_{2}\right)$ for all $f, g \in H^{*}$ and $h_{1}, h_{2} \in H$. Thus, the multiplication $m: H \otimes H \rightarrow H$ leads to the dual map $m^{*}: H^{*} \otimes H^{*} \rightarrow H^{*}$, and similarly the comultiplication $\Delta: H \rightarrow H \otimes H$ gives a map $\Delta^{*}: H^{*} \otimes H^{*} \rightarrow H^{*}$. Also, the multiplication and comultiplication give maps $\eta^{*}: H^{*} \rightarrow k^{*} \simeq k$ and $\varepsilon^{*}: k \rightarrow H^{*}$. Then all the relevant diagrams commute, making $H^{*}$ a bialgebra with multiplication $\Delta^{*}$, comultiplication $m^{*}$, unit $\varepsilon^{*}$ and counit $\eta^{*}$. Also, if $s$ is the antipode of $H$, the map $s^{*}$ is the antipode of $H^{*}$, turning $H^{*}$ into a Hopf algebra.

Hopf algebras appear in various fields of mathematics. In this thesis, the most relevant examples of Hopf algebras are the following.

Example 2.41. Let $L$ be a Lie algebra, and let $\mathcal{U} L$ be its universal enveloping algebra. Then the diagonal map $L \rightarrow L \times L, x \mapsto(x, x)$, induces a homomorphism of associative algebras $\Delta: \mathcal{U} L \rightarrow \mathcal{U} L \otimes \mathcal{U} L$. Let $\varepsilon: \mathcal{U} L \rightarrow k$ be the map induced by $L \rightarrow 0$. Then $\mathcal{U} L$ becomes a bialgebra with comultiplication $\Delta$ and counit $\varepsilon$.

By Lemma 2.24, the comultiplication is then determined by $\Delta(\iota(x))=\iota(x) \otimes 1+$ $1 \otimes \iota(x)$ for all $x \in L$. Then the anti-homomorphism $s: \mathcal{U} L \rightarrow \mathcal{U} L$ defined by $s(\iota(x))=-\iota(x)$ for all $x \in L$ satisfies $s * \mathrm{id}=\mathrm{id} * s=\eta \varepsilon$.

Example 2.42. By the above example, the polynomial $\operatorname{ring} k\left[X_{i}: i \in I\right]$ is a Hopf algebra, as it is the universal enveloping algebra of $\bigoplus_{i \in I} k$, viewed as an abelian Lie algebra. We then have, as before, $\Delta\left(X_{i}\right)=X_{i} \otimes 1+1 \otimes X_{i}$, and therefore

$$
\Delta\left(X_{i}^{n}\right)=\left(X_{i} \otimes 1+1 \otimes X_{i}\right)^{n}=\sum_{k=0}^{n}\left(\begin{array}{l}
n \\
k
\end{array}\right) X_{i}^{k} \otimes X_{i}^{n-k}
$$

for all $i \in I$. The counit $\varepsilon$ is just the evaluation at 0 , so $\varepsilon(f)=f(0)$ for all $f \in k\left[X_{i}: i \in I\right]$. 
Example 2.43. Let $k$ be a commutative ring with unit, and let $G$ be a group. The group ring $k G$ is the free $k$-module generated by the group $G$. The ring structure on $k G$ is given by the group multiplication on the generators, and extended linearly. The comultiplication and counit are given in the same way as in Example 2.34. Then $s: k G \rightarrow k G$ defined by $s(g)=g^{-1}$ for all $g \in G$ satisfies $s * \mathrm{id}=\mathrm{id} * s=\eta \varepsilon$. Example 2.42 will be of particular interest. Let $k$ be a field of characteristic $p>0$, and $k[X]$ be the polynomial ring in one variable. Then $\left(\begin{array}{c}p^{n} \\ i\end{array}\right) \equiv 0 \bmod p$ for all $0<i<p^{n}$. Therefore $\Delta\left(X^{p^{n}}\right)=X^{p^{n}} \otimes 1+1 \otimes X^{p^{n}}$ for all $n \geqslant 1$. Also $s\left(X^{p^{n}}\right)=-X^{p^{n}}$ (this holds even for $p=2$, as $-1=1$ in that case), so the ideal generated by $X^{p^{n}}$ is a Hopf ideal, turning $k[X] /\left(X^{p^{n}}\right)$ into a finite $\left(p^{n}\right.$ - $)$ dimensional Hopf algebra. Then, by the above discussion, its dual space, which we denote by $\mathcal{O}(1, \underline{n})$, is also a Hopf algebra.

Let $\left\{f_{i}: 0 \leqslant i<p^{n}\right\}$ be the basis of $\mathcal{O}(1, \underline{n})$ dual to the basis $\left\{X^{i}: 0 \leqslant i<p^{n}\right\}$ of $k[X] /\left(X^{p^{n}}\right)$, i.e. $f_{i}\left(X^{j}\right)=\delta_{i j}$. Denote by $\Delta^{*}: \mathcal{O}(1, \underline{n}) \otimes \mathcal{O}(1, \underline{n}) \rightarrow \mathcal{O}(1, \underline{n})$ the multiplication of $\mathcal{O}(1, \underline{n})$. Then

$$
\begin{aligned}
\Delta^{*}\left(f_{i} \otimes f_{j}\right)\left(X^{m}\right) & =\left(f_{i} \otimes f_{j}\right)\left(\Delta\left(X^{m}\right)\right) \\
& =\left(f_{i} \otimes f_{j}\right)\left(\sum_{k=0}^{m}\left(\begin{array}{c}
m \\
k
\end{array}\right) X^{k} \otimes X^{m-k}\right) \\
& =\sum_{k=0}^{m}\left(\begin{array}{c}
m \\
k
\end{array}\right) f_{i}\left(X^{k}\right) f_{j}\left(X^{m-k}\right)=\left\{\begin{array}{c}
\left(\begin{array}{c}
i+j \\
i
\end{array}\right), \text { if } i+j=m, \\
0 \text { otherwise. }
\end{array}\right.
\end{aligned}
$$

Therefore

$$
\Delta^{*}\left(f_{i} \otimes f_{j}\right)=\left(\begin{array}{c}
i+j \\
i
\end{array}\right) f_{i+j}
$$

The unit element of $\mathcal{O}(1, \underline{n})$ is then $\varepsilon^{*}(1)=f_{0}$, and $\mathcal{O}(1, \underline{n})$ is generated by $f_{1}$ as a $k$-algebra.

The same can be done for the polynomial ring $k\left[X_{1}, \ldots, X_{m}\right]$ in any finite number of variables. Choose any $m$-tuple $\underline{n}=\left(n_{1}, \ldots, n_{m}\right)$ of positive integers. Then the ideal generated by all the $X_{i}^{p^{n_{i}}}$ is a Hopf ideal with finite dimensional quotient (the dimension of the quotient is $p^{n_{1}} \cdots p^{n_{m}}$ ). Therefore, its dual, which we denote by $\mathcal{O}(m, \underline{n})$, is also a Hopf algebra.

For an $m$-tuple $a=\left(a_{1}, \ldots, a_{m}\right)$ of non-negative integers, we will write $\mathbf{X}^{a}$ for $X_{1}^{a_{1}} \cdots X_{m}^{a_{m}}$. Then the set of all $\mathbf{X}^{a}$ with $0 \leqslant a_{i}<p^{n_{i}}$ is a basis for $k\left[X_{1}, \ldots, X_{m}\right] /\left(X_{1}^{p^{n_{1}}}, \ldots, X_{m}^{p^{n_{m}}}\right)$.

For two $m$-tuples $a=\left(a_{1}, \ldots, a_{m}\right)$ and $b=\left(b_{1}, \ldots, b_{m}\right)$ of non-negative integers, set

$$
\left(\begin{array}{l}
a \\
b
\end{array}\right)=\prod_{i=1}^{m}\left(\begin{array}{l}
a_{i} \\
b_{i}
\end{array}\right) \text { and } a+b=\left(a_{1}+b_{1}, \ldots, a_{m}+b_{m}\right) \text {. }
$$

Then, if $\left\{\mathbf{X}^{(a)}: 0 \leqslant a_{i}<p^{n_{i}}\right\}$ is the basis of $\mathcal{O}(m, \underline{n})$ dual to $\left\{\mathbf{X}^{a}: 0 \leqslant a_{i}<p^{n_{i}}\right\}$, the multiplication rule in $\mathcal{O}(m, \underline{n})$ is

$$
\mathbf{X}^{(a)} \mathbf{X}^{(b)}=\left(\begin{array}{c}
a+b \\
a
\end{array}\right) \mathbf{X}^{(a+b)}
$$


By [46, Lemma 2.1.2], we have $\left(\begin{array}{c}p j \\ j\end{array}\right) \equiv 0 \bmod p$ for $j>0$. It follows that for $a \neq(0, \ldots, 0)$, we have

$$
\left(\mathbf{X}^{(a)}\right)^{p}=\left(\begin{array}{c}
p a \\
a
\end{array}\right) \mathbf{X}^{(p a)}=0
$$

Set $\underline{1}=(1, \ldots, 1)$. Then the map $\psi: k\left[X_{1}, \ldots, X_{m}\right] /\left(X_{1}^{p}, \ldots, X_{m}^{p}\right) \rightarrow \mathcal{O}(m, \underline{1}), \mathbf{X}^{a} \mapsto$ $a ! \mathbf{X}^{(a)}$ is an isomorphism of associative algebras, as it is clearly bijective and

$$
\psi\left(\mathbf{X}^{a}\right) \psi\left(\mathbf{X}^{b}\right)=a ! b ! \mathbf{X}^{(a)} \mathbf{X}^{(b)}=a ! b !\left(\begin{array}{c}
a+b \\
a
\end{array}\right) \mathbf{X}^{(a+b)}=(a+b) ! \mathbf{X}^{(a+b)}=\psi\left(\mathbf{X}^{a+b}\right) .
$$

Theorem 2.44. For $\underline{n}=\left(n_{1}, \ldots, n_{m}\right)$, let $|\underline{n}|=\sum_{i} n_{i}$. Then there is an isomorphism

$$
\mathcal{O}(m, \underline{n}) \simeq \mathcal{O}(|\underline{n}|, \underline{1})
$$

Proof. We have $\mathcal{O}(|\underline{n}|, 1)=k\left[X_{1}, \ldots, X_{n_{1}}, X_{n_{1}+1}, \ldots, X_{n_{1}+n_{2}}, \ldots, X_{|\underline{n}|}\right] /\left(X_{i}^{p}\right)$. Let $X_{1}^{(1)}, \ldots, X_{m}^{(m)}$ be the generators of $\mathcal{O}(m, \underline{n})$. Define

$$
\begin{aligned}
\phi: \mathcal{O}(|\underline{n}|, \underline{1}) & \rightarrow \mathcal{O}(m, \underline{n}), \\
X_{1} & \mapsto X_{1}^{(1)}, \\
X_{2} & \mapsto X_{1}^{(p)}, \\
X_{3} & \mapsto X_{1}^{(2 p)}, \\
\vdots & \\
X_{n_{1}} & \mapsto X_{1}^{\left(\left(n_{1}-1\right) p\right)}, \\
X_{n_{1}+1} & \mapsto X_{2}^{(1)}, \\
\vdots & \\
X_{|\underline{n}|} & \mapsto X_{m}^{\left(n_{m}-1\right) p} .
\end{aligned}
$$

This map is well defined by the above remark, and clearly injective. As $\operatorname{dim}(\mathcal{O}(m, \underline{n}))=$ $p^{|\underline{n}|}=\operatorname{dim}(\mathcal{O}(|\underline{n}|, \underline{1}))$, it follows that $\phi$ is an isomorphism.

\subsection{Simple and Semisimple Lie Algebras}

Definition 2.45. A non-abelian Lie algebra $L$ is called simple, if the only two ideals of $L$ are 0 and $L$.

Furthermore, a Lie algebra $L$ is said to be semisimple, if 0 is the only abelian ideal of $L$.

Example 2.46. Let $k$ be a field with $\operatorname{char}(k) \neq 2$. Set

$$
\mathfrak{s l}_{2}(k)=\left\{\left(\begin{array}{ll}
a & b \\
c & d
\end{array}\right) \in k^{2 \times 2}: a+d=0\right\} .
$$

Then $\mathfrak{s l}_{2}(k)$ is a simple Lie algebra.

Indeed, set $e=\left(\begin{array}{ll}0 & 1 \\ 0 & 0\end{array}\right), f=\left(\begin{array}{ll}0 & 0 \\ 1 & 0\end{array}\right)$ and $h=\left(\begin{array}{cc}1 & 0 \\ 0 & -1\end{array}\right)$. Then the set $\{e, f, h\}$ is a 
basis of $\mathfrak{s l}_{2}(k)$, and their commutators are $[e, f]=h,[h, e]=2 e$ and $[h, f]=-2 f$. Let now $I \subseteq \mathfrak{s l}_{2}(k)$ be a non-zero ideal. Note first that if $h \in I$, then $1 / 2[h, e]=$ $e \in I$ and $-1 / 2[h, f]=f \in I$, and therefore $I=\mathfrak{s l}_{2}(k)$.

Let $x=x_{e} e+x_{f} f+x_{h} h \in I$ be a non-zero element. Then

$$
\begin{aligned}
{[x, e] } & =-x_{f} h+2 x_{h} e \in I, \\
{[x, f] } & =x_{e} h-2 x_{h} f \in I \text { and } \\
{[x, h] } & =-2 x_{e} e+2 x_{f} f \in I .
\end{aligned}
$$

If $x_{e}=0$, then $x_{f} \neq 0$ or $x_{h} \neq 0$. In both cases we conclude $f \in I$, and therefore $[f, e]=h \in I$, thus $I=\mathfrak{s l}_{2}(k)$. If $x_{f}=0$, we similarly conclude $h \in I$. So assume now $x_{e}, x_{f} \neq 0$. Then $[[x, h], f]=-2 x_{e} h \in I$, and therefore again $h \in I$.

It is clear from the definition that every simple Lie algebra is semisimple. Also, if $L_{1}$ and $L_{2}$ are semisimple, their direct sum $L_{1} \oplus L_{2}$ is semisimple, for if $I \subseteq L_{1} \oplus L_{2}$ is an abelian ideal, its respective projections to $L_{1}$ and $L_{2}$ are abelian ideals of $L_{1}$ and $L_{2}$, and thus zero. Therefore $I=0$. However, if $L_{1}$ and, $L_{2}$ are simple (and therefore non-zero), then $L_{1}$ and $L_{2}$ are ideals of $L_{1} \oplus L_{2}$, so $L_{1} \oplus L_{2}$ is not simple.

Theorem 2.47 (Structure Theorem, e.g. [22, p.71]). A finite dimensional Lie algebra L over a field of characteristic zero is semisimple, if and only if

$$
L \simeq \bigoplus_{i=1}^{r} L_{i}
$$

with simple Lie algebras $L_{i}$.

By the Structure Theorem, characterizing all finite dimensional semisimple Lie algebras over a field of characteristic zero reduces to characterizing the finite dimensional simple Lie algebras over that field.

Definition 2.48. Let $L$ be a Lie algebra. A Cartan subalgebra is a nilpotent subalgebra $H \subseteq L$ which is self-normalizing, i.e. if $[x, y] \in H$ for all $x \in H$, then $y \in H$.

Example 2.49. Using the notation of Example 2.46, $H:=\operatorname{span}(h)$ is a Cartan subalgebra of $\mathfrak{s l}_{2}(k)$. Indeed, $H$ is clearly abelian, and thus nilpotent. Also, if $y=y_{e} e+y_{f} f+y_{h} h \in \mathfrak{s l}_{2}(k)$, such that $[x, y] \in H$ for all $x \in H$, then $[h, y]=$ $2 y_{e} e-2 y_{f} f \in H$, so $y+e=y_{f}=0$ and thus $y \in H$.

Theorem 2.50 ([22, Chapter IV]). Let L be a semisimple Lie algebra over an algebraically closed field of characteristic zero. Then

1. There exists a Cartan subalgebra $H$ of $L$,

2. $H$ is unique up to automorphism,

3. $H$ is abelian and

4. $\operatorname{ad}_{h} \in \operatorname{End}(L)$ is diagonalizable for every $h \in H$. 
The study of Cartan subalgebras plays an important role in classifying the simple Lie algebras over algebraically closed fields of characteristic zero. One can define a non-degenerate bilinear form on the dual space $H^{*}$, such that classifying simple Lie algebras reduces to classifying so called root systems, which are very geometrical objects, and corresponding Dynkin diagrams, which are graphs. The finite dimensional simple Lie algebras over an algebraically closed field of characteristic zero can then be completely classified.

Theorem 2.51 ([22, Chapter IV]). Every simple Lie algebra L over an algebraically closed field of characteristic zero is of one of the following forms:

1. $\mathfrak{s l}_{n}(k)$ with $n \geqslant 2$,

2. $\mathfrak{s o}_{n}(k)$ with $n \geqslant 3$ and $n \neq 4$,

3. $\mathfrak{s p}_{2 n}(k)$ with $n \geqslant 4$ or

4. one of the exceptional Lie algebras $\mathfrak{e}_{6}, \mathfrak{e}_{7}, \mathfrak{e}_{8}, \mathfrak{f}_{4}$ or $\mathfrak{g}_{2}$.

If the characteristic of the base field $k$ is positive, studying the finite dimensional simple Lie algebras over that field becomes slightly more complicated. However, all the Lie algebras listed above admit a certain basis, called the Chevalley basis, such that the structure constants with respect to that basis are integers. For a simple Lie algebra $L$ as above, these structure constants give a Lie algebra $L_{\mathbb{Z}}$ over $\mathbb{Z}$. Then $L_{k}:=k_{\mathbb{Z}} \otimes F$ is a Lie algebra over $k$.

Theorem 2.52 ([42]). Let $k$ be a field with $\operatorname{char}(k)=p>3$, and let $L$ be a finite dimensional simple Lie algebra over a field of characteristic zero. Let $L_{k}$ be as above. Then $L_{k}$ is simple, except when $L=\mathfrak{s l}_{n}(k)$ with $p \mid n$. In that case, $L_{k} / Z\left(L_{k}\right)$ is simple.

However, the list given in Theorem 2.51 is not complete if the ground field is of positive characteristic, as the following example shows.

Example 2.53. Let $k$ be a field with $\operatorname{char}(k) \geqslant 5$. Then the Lie algebra $W(1, \underline{1}):=$ $\operatorname{Der}\left(k[X] /\left(X^{p}\right)\right)$ is simple but not isomorphic to either of the above Lie algebras. Indeed, if we set $e_{i}=X^{i+1} d / d X$, the set $\left\{e_{i}:-1 \leqslant i \leqslant p-2\right\}$ is a basis of $W(1, \underline{1})$ with the multiplication rule

$$
\left[e_{i}, e_{j}\right]= \begin{cases}(j-i) e_{i+j}, & \text { if }-1 \leqslant i+j \leqslant p-2 \\ 0 & \text { else. }\end{cases}
$$

Now suppose $I \subseteq W(1, \underline{1})$ is a non-zero ideal. Let $D=\sum_{i=-1}^{p-2} \lambda_{i} e_{i} \in I$, and suppose $j$ is the maximal index with $\lambda_{j} \neq 0$.

Suppose first $j \neq-1$, then $I \ni\left[D, e_{p-2-j}\right]=-2 \lambda_{j} e_{p-2}$, therefore $e_{p-2} \in I$. It follows that $\left[e_{p-2}, e_{-1}\right]=e_{p-3} \in I,\left[e_{p-3}, e_{-1}\right]=2 e_{p-4} \in I$, and so on. Thus $e_{i} \in I$ for all $i$, and $I=W(1, \underline{1})$.

If $j=-1$, then $D=\lambda_{-1} e_{-1}$, so assume without loss of generality that $\lambda_{-1}=1$, so $D=e_{-1} \in I$. Then $\left[e_{1}, e_{-1}\right]=2 e_{0} \in I$, thus $e_{0} \in I$. Then $\left[e_{0}, e_{i}\right]=i e_{i} \in I$ for 
every $i$, thus $e_{i} \in I$ for every $i$, and $I=W(1, \underline{1})$.

As $\operatorname{dim}(W(1, \underline{1}))=p \geqslant 5$, it cannot be of either of the classes given in Theorem 2.51, as the only one of those whose dimension is a prime number is the 3-dimensional Lie algebra $\mathfrak{s l}_{2}(K)$ (which is isomorphic to $W(1, \underline{1})$ if $\operatorname{char}(k)=3$ ). The Lie algebra $W(1, \underline{1})$ is often called the Witt algebra. This example can be further generalized to the generalized Jacobson-Witt algebras. Let $k$ be a field of characteristic $p>3$, and for $m \in \mathbb{N}$ and $\underline{n}=\left(n_{1}, \cdots, n_{m}\right) \in \mathbb{N}^{m}$ denote by $\mathcal{O}(m, \underline{n})$ the Hopf dual of $k\left[X_{1}, \ldots, X_{m}\right] /\left(X_{1}^{p^{n_{1}}}, \ldots, X_{m}^{p^{n_{m}}}\right)$ as above. Set $e_{i}=$ $(0, \ldots, 0,1,0, \ldots, 0)$ with the 1 in the $i$-th position, and for convenience we set $\mathbf{X}^{(a)}=0$ if $a_{i}<0$ for some $i$.

Definition 2.54. With the notation as above, we call a derivation $D$ of $\mathcal{O}(m, \underline{n})$ special, if

$$
D\left(\mathbf{X}^{(a)}\right)=\sum_{i=1}^{m} \mathbf{X}^{\left(a-e_{i}\right)} D\left(\mathbf{X}^{\left(e_{i}\right)}\right)
$$

for all $a=\left(a_{1}, \ldots, a_{m}\right)$ with $0 \leqslant a_{i}<p^{n_{i}}$. Then the generalized Jacobson-Witt algebra $W(m, \underline{n})$ is the set of all special derivations of $\mathcal{O}(m, \underline{n})$.

In other words, if we denote by $\partial_{i}$ the $i$-th partial derivative, i.e. $\partial_{i}\left(\mathbf{X}^{(a)}\right)=\mathbf{X}^{\left(a-e_{i}\right)}$, it can be shown that $W(m, \underline{n})$ is indeed a Lie algebra with

$$
W(m, \underline{n})=\bigoplus_{i=1}^{m} \mathcal{O}(m, \underline{n}) \partial_{i},
$$

and $W(m, \underline{n})=\operatorname{Der}(\mathcal{O}(m, \underline{n}))$ if and only if $\underline{n}=\underline{1}$ (see [47, Chapter 3, Proposition $5.9])$.

Theorem 2.55 ([47, Chapter 4, Theorem 2.4]). The Lie algebras $W(m, \underline{n})$ are always simple if $p \geqslant 3$, and not isomorphic to any of the Lie algebras from Theorem 2.51 (except for $p=3$ and $\underline{n}=1$, as mentioned above).

Some further classes of finite dimensional simple Lie algebras over fields of positive characteristic occur as subalgebras of $W(m, \underline{n})$. Though they are not relevant in this thesis, we define them here for completion.

Define the map

$$
\partial_{i j}: \begin{cases}\mathcal{O}(m, \underline{n}) & \rightarrow W(m, \underline{n}) \\ f & \mapsto \partial_{j}(f) \partial_{i}-\partial_{i}(f) \partial_{j} .\end{cases}
$$

Then one easily sees that $\partial_{i i}=0$ and $\partial_{i j}=-\partial_{j i}$ for all $1 \leqslant i, j \leqslant m$. Furthermore, $\partial_{i j}$ is $k$-linear, with $\left[\partial_{\ell}, \partial_{i j}(f)\right]=\partial_{i j}\left(\partial_{\ell}(f)\right)$ for all $f \in \mathcal{O}(m, \underline{n})$ and $1 \leqslant \ell \leqslant m$. Then the set

$$
S(m, \underline{n}):=\left\langle\left\{\partial_{i j}(f): f \in \mathcal{O}(m, \underline{n}), 1 \leqslant i<j \leqslant m\right\}\right\rangle
$$

is another finite dimensional simple Lie algebra (of dimension $(m-1) p^{|\underline{n}|}-1$ ) over $k$, called the special algebra.

Furthermore, if $m=2 r$ is even, for $i \in\{1, \ldots, m\}$ set

$$
\sigma(i)= \begin{cases}1, & \text { if } i \leqslant r \\ -1, & \text { if } i>r .\end{cases}
$$


Then we can define another map

$$
\partial_{H}: \begin{cases}\mathcal{O}(m, \underline{n}) & \rightarrow W(m, \underline{n}) \\ f & \mapsto \sum_{i=1}^{m} \sigma(i) \partial_{i}(f) \partial_{i} .\end{cases}
$$

Then the set

$$
H(m, \underline{n}):=\left\langle\partial_{H}\left(\mathbf{X}^{(a)}\right): 0 \leqslant a_{i}<p^{n_{i}}\right\rangle
$$

is a finite dimensional simple Lie algebra of dimension $p^{|\underline{n}|}-2$, called the hamiltonian algebra.

Finally, for $m=2 r+1$ uneven, for $j \leqslant 2 r$ set

$$
j^{\prime}= \begin{cases}j+r, & \text { if } j \leqslant r \\ j-r, & \text { if } j>r .\end{cases}
$$

Then, for $f \in \mathcal{O}(m, \underline{n})$ and $j \leqslant 2 r$, set

$$
f_{j}=\mathbf{X}^{\left(e_{j}\right)} \partial_{m}(f)+\sigma\left(j^{\prime}\right) \partial_{j^{\prime}}(f),
$$

where $\sigma$ is defined as above. Also set

$$
f_{m}=2 f-\sum_{j=1}^{2 r} \sigma(j) \mathbf{X}^{\left(e_{j}\right)} f_{j} .
$$

Then define a map

$$
\partial_{K}: \begin{cases}\mathcal{O}(m, \underline{n}) & \rightarrow W(n, \underline{m}) \\ f & \mapsto \sum_{j=1}^{m} f_{j} \partial_{j} .\end{cases}
$$

Then for $f, g \in \mathcal{O}(m, \underline{n})$, we set $\langle f, g\rangle=\partial_{K}(f)(g)-2 \partial_{m}(f)$. Then one can show that $\langle\cdot, \cdot\rangle$ defines a Lie bracket on $\mathcal{O}(m, \underline{n})$. Now if $n \not \equiv-3 \bmod p$, then $\mathcal{O}(m, \underline{n})$ becomes a simple Lie algebra of dimension $p^{|\underline{n}|}$ with this operation, denoted by $K(m, \underline{n})$. If on the other hand $n \equiv-3 \bmod p$, the set $\operatorname{span}\left\{\mathbf{X}^{(a)}: \exists i: a_{i}<p^{n_{i}}-1\right\}$, also denoted by $K(m, \underline{n})$ is a simple Lie algebra of dimension $p^{|\underline{n}|}-1$. These Lie algebras are normally referred to as contact algebras.

\subsection{Simplicial Objects}

Definition 2.56. The simplicial category $\Delta$ is the category defined in the following way. The objects are the sets $[n]:=\{0, \ldots, n\}$ for $n \in \mathbb{N}_{0}$, and a map $f:[n] \rightarrow[m]$ is a morphism, if and only if $f$ is order preserving, i.e. $f(i) \leqslant f(j)$, whenever $i \leqslant j$.

In particular, for each $n$ the maps

$$
\delta_{i}:[n-1] \rightarrow[n], j \mapsto \begin{cases}j, & \text { if } j<i, \\ j+1, & \text { if } j \geqslant i\end{cases}
$$

and

$$
\sigma_{i}:[n+1] \rightarrow[n], j \mapsto \begin{cases}j, & \text { if } j \leqslant i \\ j-1, & \text { if } j>i\end{cases}
$$


for $0 \leqslant i \leqslant n$ are morphisms of $\Delta$. In fact, it is shown in [32, p. 4], that the $\delta_{i}$ and $\sigma_{i}$ generate the morphisms of $\Delta$. They satisfy the equations

$$
\begin{aligned}
\delta_{j} \delta_{i} & =\delta_{i} \delta_{j-1}, \text { if } i<j, \\
\sigma_{j} \sigma_{i} & =\sigma_{i} \sigma_{j+1}, \text { if } i \leqslant j \\
\sigma_{j} \delta_{i} & = \begin{cases}\delta_{i} \sigma_{j-1}, & \text { if } i<j \\
\mathrm{id}, & \text { if } i=j, j+1, \\
\delta_{i-1} \sigma_{j}, & \text { if } i>j+1 .\end{cases}
\end{aligned}
$$

Definition 2.57. Let $\mathcal{C}$ be any category. A simplicial object in $\mathcal{C}$ is a contravariant functor $F: \Delta \rightarrow \mathcal{C}$. In other words, a simplicial object is a collection $\mathbf{X}=\left\{X_{n}\right\}_{n \geqslant 0}$ of objects in $\mathcal{C}$ together with morphisms $d_{i}: X_{n} \rightarrow X_{n-1}$ and $s_{i}: X_{n} \rightarrow X_{n+1}$ for each $n$ and $0 \leqslant i \leqslant n$, satisfying

$$
\begin{aligned}
& d_{i} d_{j}=d_{j-1} d_{i}, \text { if } i<j, \\
& s_{i} s_{j}=s_{j+1} s_{i}, \text { if } i \leqslant j, \\
& d_{i} s_{j}= \begin{cases}s_{j-1} d_{i}, & \text { if } i<j, \\
\mathrm{id}, & \text { if } i=j, j+1, \\
s_{j} d_{i-1}, & \text { if } i>j+1 .\end{cases}
\end{aligned}
$$

The maps $d_{i}$ will be called face maps, and the $s_{i}$ will be called degeneracy maps.

Example 2.58. Let $\Delta_{n}=\left\{\left(x_{0}, \ldots, x_{n}\right) \in \mathbb{R}^{n+1}: 0 \leqslant x_{i} \leqslant 1, \sum_{i} x_{i}=1\right\}$. Then $\left\{\Delta_{n}\right\}_{n \geqslant 0}$ is a simplicial topological space with face maps

$$
d_{i}: \Delta_{n} \rightarrow \Delta_{n-1},\left(x_{0}, \ldots, x_{n}\right) \mapsto\left(x_{0}, \ldots, x_{i}+x_{i+1}, \ldots, x_{n}\right)
$$

and degeneracy maps

$$
s_{i}: \Delta_{n} \rightarrow \Delta_{n+1},\left(x_{0}, \ldots, x_{n}\right) \mapsto\left(x_{0}, \ldots, x_{i}, 0, x_{i+1}, \ldots, x_{n}\right) .
$$

Definition 2.59. Let $\mathbf{X}=\left\{X_{n}\right\}_{n \geqslant 0}$ and $\mathbf{Y}=\left\{Y_{n}\right\}_{n \geqslant 0}$ be simplicial objects in a category $\mathcal{C}$. A morphism $\boldsymbol{\phi}: \mathbf{X} \rightarrow \mathbf{Y}$ is a collection of maps $\left\{\phi_{n}: X_{n} \rightarrow Y_{n}\right\}_{n \geqslant 0}$, such that all the diagrams

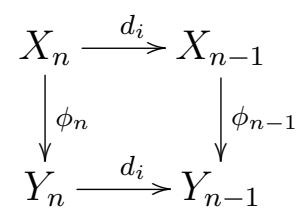

and

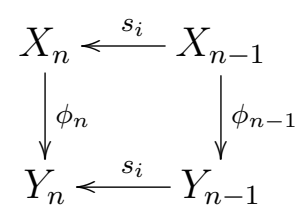

commute. In this way, the class of simplicial objects in $\mathcal{C}$ becomes itself a category, which we will denote by $\mathcal{S C}$. 
Definition 2.60. Let $\mathrm{X}$ and $\mathrm{Y}$ be two simplicial objects in a category $\mathcal{C}$, and $\boldsymbol{\phi}, \boldsymbol{\psi}: \mathrm{X} \rightarrow \mathrm{Y}$ be morphisms between them. A homotopy between $\boldsymbol{\phi}$ and $\boldsymbol{\psi}$ is a collection of maps $h_{i}: X_{n} \rightarrow Y_{n+1}, 0 \leqslant i \leqslant n$, for each $n \geqslant 0$, satisfying

$$
\begin{aligned}
& d_{0} h_{0}=\phi_{n}, d_{n+1} h_{n}=\psi_{n+1} \\
& d_{i} h_{j}= \begin{cases}h_{j-1} d_{i}, & \text { if } i<j, \\
d_{i} h_{i-1}, & \text { if } i=j \neq 0, \\
h_{j} d_{i-1}, & \text { if } i>j+1,\end{cases} \\
& s_{i} h_{j}= \begin{cases}h_{j+1} s_{i}, & \text { if } i \leqslant j, \\
h_{j} s_{i-1}, & \text { if } i>j .\end{cases}
\end{aligned}
$$

The maps $\boldsymbol{\phi}$ and $\boldsymbol{\psi}$ are called homotopic, if there exists a homotopy between them.

Definition 2.61. Two simplicial objects $\mathbf{X}$ and $\mathbf{Y}$ are said to be of the same homotopy type, if there exist simplicial morphisms $\phi: \mathbf{X} \rightarrow \mathbf{Y}$ and $\boldsymbol{\psi}: \mathbf{Y} \rightarrow \mathbf{X}$, such that $\boldsymbol{\phi} \circ \boldsymbol{\psi}$ and $\boldsymbol{\psi} \circ \boldsymbol{\phi}$ are homotopic to the respective identity morphisms.

If $\mathcal{C}$ is the category of groups, where the identity element of each group is denoted by 0 . Then every simplicial group $\mathbf{X}$ gives rise to a chain complex of groups in the following way.

Definition 2.62. The Moore complex $\mathbf{M}(\mathbf{X})=\left\{M_{n}(\mathbf{X})\right\}_{n \geqslant 0}$ of a simplicial group $\mathrm{X}$ is the complex with

$$
M_{n}(\mathbf{X})=\bigcap_{i=0}^{n-1} \operatorname{ker}\left(d_{i}: X_{n} \rightarrow X_{n-1}\right)
$$

and differentials $\left.d_{n}\right|_{M_{n}(\mathbf{X})}: M_{n}(\mathbf{X}) \rightarrow M_{n-1}(\mathbf{X})$. We will write only $M_{n}$ instead of $M_{n}(\mathbf{X})$, when there is no danger of confusion.

Proposition 2.63. The Moore complex is indeed a chain complex, and $d_{n}\left(M_{n}\right)$ is a normal subgroup of $X_{n-1}$, so in particular of $M_{n-1}$, for every $n \geqslant 1$.

Proof. Let $x \in M_{n}$. Then $d_{i}(x)=1$ for all $i<n$. Thus, by (2.11), $d_{i} d_{n}(x)=$ $d_{n-1} d_{i}(x)=d_{n-1}(0)=0$ for all $i<n-1$, and therefore $d_{n}(x) \in M_{n-1}$. Also by (2.11), $d_{n-1} d_{n}(x)=d_{n-1} d_{n-1}(x)=d_{n-1}(0)=0$, so $d_{n-1} d_{n}$ is the zero map.

Also, let $y=d_{n}(x) \in d_{n}\left(M_{n}\right) \subseteq M_{n-1}$ with $x \in M_{n}$, and let $a \in X_{n-1}$. Note that by 2.13 we have $a=d_{n} s_{n-1}(a)$. Therefore $a^{-1} y a=d_{n}\left(s_{n-1}(a)^{-1} x s_{n-1}(a)\right)$, and as $M_{n}$ is normal in $X_{n}$, it follows that $s_{n-1}(a)^{-1} x s_{n-1}(a) \in M_{n}$, and therefore $a^{-1} y a \in d_{n}\left(M_{n}\right)$ as desired.

Definition 2.64. Let $\mathbf{X}$ be a simplicial group and $n \geqslant 0$. Then the $n$-th homotopy group of $\mathbf{X}$ is defined as

$$
\pi_{n}(\mathbf{X})=\operatorname{ker}\left(\left.d_{n}\right|_{M_{n}}\right) / d_{n+1}\left(M_{n+1}\right) .
$$


Every morphism $\phi: \mathbf{X} \rightarrow \mathbf{Y}$ of simplicial groups induces morphisms $\phi_{*}: \pi_{n}(\mathbf{X}) \rightarrow$ $\pi_{n}(\mathbf{Y})$ for every $n \geqslant 0$ given by $\phi_{*}\left(x d_{n+1}\left(M_{n+1}(\mathbf{X})\right)\right)=\phi_{n}(x) d_{n+1}\left(M_{n+1}(\mathbf{Y})\right)$. Thus $\pi_{n}$ is a covariant functor from the category of simplicial groups to the category of groups for every $n \geqslant 0$. In fact, by [32, Proposition 17.3], $\pi_{n}(\mathbf{X})$ is abelian for all $n \geqslant 1$, therefore in these cases, $\pi_{n}$ is actually a functor into the category of abelian groups.

Proposition 2.65 ([32, Theorem 22.1]). Let $\mathbf{A}=\left\{A_{n}\right\}_{n \geqslant 0}$ be a simplicial abelian group. Define the total differentials

$$
\partial_{n}:=\sum_{i=0}^{n}(-1)^{i} d_{i}: A_{n} \rightarrow A_{n-1}
$$

for $n \geqslant 0$ (where we set $A_{-1}=0$ and $\partial_{0}$ the zero-morphism).

Then $\partial_{n-1} \circ \partial_{n}=0$, and the inclusion $M_{n}(\mathbf{A}) \hookrightarrow A_{n}$ induces an isomorphism between $\pi_{n}(\mathbf{A})$ and the $n$-th homology group of the resulting chain complex.

\subsection{The Dimension Subgroup Problem}

For a group $G$ and a ring with unit $k$, one can define the group ring $k G$ as the free $k$-module with basis $G$, whose multiplication rule is inherited from the group operation in $G$ and extended linearly. Then $k G$ is also a ring with unit (the unit element is $1 \cdot e$, where 1 is the unit of $k$ and $e$ the identity element of $G$ ), which is commutative if and only if both $k$ and $G$ are. Write 1 for $1 \cdot e$.

Now the question we may ask is: How are the normal subgroups of $G$ and the ideals of $k G$ connected? Let $H$ be a normal subgroup of $G$. Then one easily verifies that $k G(H-1)$ is an ideal of $k G$. Thus we get a map

$$
I: \begin{cases}\{\text { normal subgroups of } G\} & \rightarrow\{\text { ideals of } k G\}, \\ H & \mapsto k G(H-1) .\end{cases}
$$

Note that, as $H$ is normal in $G$, so $g H=H g$ for every $g \in G$, this indeed defines a two-sided ideal of $k G$.

On the other hand, for any two sided ideal $\mathfrak{a} \subseteq k G$, the set $G \cap(1+\mathfrak{a})=: D_{k}(\mathfrak{a})$ defines a normal subgroup of $G$. Therefore, we also have a map

$$
D_{k}: \begin{cases}\{\text { ideals of } k G\} & \rightarrow\{\text { normal subgroups of } G\}, \\ \mathfrak{a} & \mapsto D_{k}(\mathfrak{a}) .\end{cases}
$$

The groups $D_{k}(\mathfrak{a})$ will be called generalized dimension subgroups of $\mathfrak{a}$. One can now wonder whether the two maps are inverses of each other, or more generally how they are connected.

Proposition 2.66. With the notation as above, we have

$$
D_{k}(I(H))=H
$$

for every normal subgroup $H \subseteq G$. 
Proof. First observe that if $h \in H$, then obviously $h \in G$ and $h-1 \in k G(H-1)$, so $h \in 1+k G(H-1)$, and thus $h \in D_{k}(I(H))$, so $H \subseteq D_{k}(I(H))$.

For the reverse inclusion, note that $k G(H-1)$ is the kernel of the homomorphism $\pi^{*}: k G \rightarrow k(G / H)$ induced by the natural projection $\pi: G \rightarrow G / H$. Thus, if $g \in D_{k}(I(H))$, then $0=\pi^{*}(g-1)=g H-1$, and therefore $g H=H$ and therefore $g \in H$.

As there are many more ideals in $k G$ than just those induced by some subgroup of $G$, it is not particularly surprising that the other composition is not the identity. Take e.g. the ideals $k G(G-1)=: \varpi_{k}(G)$ and the trivial ideal $k G$. Both induce the same subgroup, namely $G$, but the latter one is not induced by any subgroup. Let us look at some examples to get a feel for it. First, let $G=C_{p}$ be the cyclic group of order $p$, and let $k=\mathbb{F}_{p}$ be the field with $p$ elements. Then $k G \simeq$ $k[X] /\left\langle X^{p}-1\right\rangle=k[X] /\left\langle(X-1)^{p}\right\rangle$. Therefore the ideals of $k G$ correspond to ideals $\mathfrak{a} \subseteq k[X]$ with $(X-1)^{p} \in \mathfrak{a}$. As $k[X]$ is a principal ideal domain, these are given by $\left\langle(X-1)^{i}\right\rangle$ with $0 \leqslant i \leqslant p$. Thus all ideals are powers of the augmentation ideal, and $\varpi_{k}^{p}(G)=0$.

Now, if $G=C_{p}=\langle g\rangle$ as above, and $k$ is a field not containing a primitive $p$-th root of unity, then by the Chinese Remainder Theorem

$k G \simeq k[X] /\left\langle X^{p}-1\right\rangle \simeq k[X] /\langle X-1\rangle \oplus k[X] /\left\langle X^{p-1}+X^{p-2}+\cdots+X+1\right\rangle \simeq k \oplus k\left(\zeta_{p}\right)$,

where $\zeta_{p}$ denotes a primitive $p$-th root of unity in an algebraic closure of $k$. The isomorphism between $k G$ and $k \oplus k\left(\zeta_{p}\right)$ is given by $g \mapsto\left(1, \zeta_{p}\right)$. Now, as $k G$ is a direct sum of two fields, the only non-trivial ideals are given by its direct summands. One is the augmentation ideal, corresponding to the ideal $0 \oplus k\left(\zeta_{p}\right)$ in the isomorphic image, and the other is the ideal generated by the norm element $N:=\sum_{i=0}^{p-1} g^{i}$.

As another example, let $G$ be the alternating group $A_{5}$, which is known to be simple. Therefore, the only normal subgroups of $A_{5}$ are $\{e\}$ and $A_{5}$. Now let $k$ be an algebraically closed field with $\operatorname{char}(k) \neq 2,3,5$. Then by Maschke's Theorem (see e.g. [25, Chapter XVIII, §1, Theorem 1]) $k G$ is a semisimple $k$-algebra, so by the Artin-Wedderburn-Theorem (see e.g. [25, Chapter XVII]) we get

$$
k G \simeq \bigoplus_{i=1}^{r} k^{n_{i} \times n_{i}}
$$

where $r$ is the number of conjugacy classes of $A_{5}$, which happens to be 5 . Now, as $k^{n_{i} \times n_{i}}$ is a simple $k$-algebra, all the 5 direct summands of the decomposition above and sums of those are the ideals of $k G$. So, in all, the number of ideals of $k G$ is equal to $2^{5}=32$.

The general problem is now, for a given ideal $\mathfrak{a} \subseteq k G$, to identify the subgroup $D_{k}(\mathfrak{a})$. The following Lemma shows how the ideals behave under the group multiplication and the group commutator.

Lemma 2.67. Let $\mathfrak{a}, \mathfrak{b} \subseteq k G$ be two ideals of a group ring $k G$. Then

$$
\begin{aligned}
D_{k}(\mathfrak{a}) D_{k}(\mathfrak{b}) & \subseteq D_{k}(\mathfrak{a}+\mathfrak{b}) \text { and } \\
{\left[D_{k}(\mathfrak{a}), D_{k}(\mathfrak{b})\right] } & \subseteq D_{k}(\mathfrak{a} \mathfrak{b}+\mathfrak{b} \mathfrak{a}) .
\end{aligned}
$$


Proof. Let $a \in D(k, \mathfrak{a})$ and $b \in D(k, \mathfrak{b})$, i.e. $a-1 \in \mathfrak{a}$ and $b-1 \in \mathfrak{b}$. Then

$$
a b-1=(a-1)(b-1)+(a-1)+(b-1) \in \mathfrak{a}+\mathfrak{b},
$$

and thus $a b \in D_{k}(\mathfrak{a}+\mathfrak{b})$, proving the first assertion.

Also, as

$$
[a, b]-1=a^{-1} b^{-1}((a-1)(b-1)-(b-1)(a-1)),
$$

we see that $[a, b] \in D(k, \mathfrak{a} \mathfrak{b}+\mathfrak{b} \mathfrak{a})$, completing the proof.

Let $\varepsilon: k G \rightarrow k$ be the ring homomorphism defined by $g \mapsto 1$ for all $g \in G$. Then $\operatorname{ker}(\varepsilon)=k G(G-1)=\varpi_{k}(G)$. As an $k$-module, $\varpi_{k}(G)$ is generated by all elements $g-1$ for $g \in G$. Then the dimension subgroup problem is to identify the subgroup $\delta_{n, k}(G):=D_{k}\left(\varpi_{k}^{n}(G)\right)$. For simplicity we will omit the index $k$ if $k=\mathbb{Z}$. For example, we write $\delta_{n}(G)$ for $\delta_{n, \mathbb{Z}}(G)$.

Denote by $\left\{\gamma_{n}(G)\right\}_{n \geqslant 1}$ the lower central series of a group $G$ as defined in (2.3). Then by induction on $n$ and Lemma 2.67 one easily sees that $\gamma_{n}(G) \subseteq \delta_{n, k}(G)$ for all $n$ and for all rings $k$. Furthermore, the above equation shows that the series $\left\{\delta_{n, k}(G)\right\}_{n \geqslant 1}$ satisfies

$$
\left[\delta_{n, k}(G), \delta_{m, k}(G)\right] \subseteq \delta_{n+m, k}(G)
$$

for every group $G$, ring $k$ and $n, m \geqslant 1$.

As we have to deal with descending series of groups, we introduce some terminology about such series.

Definition 2.68. Let $G$ be a group, and

$$
G=G_{1} \supseteq G_{2} \supseteq G_{3} \supseteq \cdots
$$

a descending series of normal subgroups. The series is called

- central series, if $\left[G, G_{i}\right] \subseteq G_{i+1}$ for every $i \geqslant 1$,

- $N$-series, if $\left[G_{i}, G_{j}\right] \subseteq G_{i+j}$ for all $i, j \geqslant 1$ and

- $N_{0}$-series, if it is an $N$-series and $G_{i} / G_{i+1}$ is torsion free for every $i \geqslant 1$.

It is clear from the definition that every $N_{0}$-series is an $N$-series and every $N$-series is a central series. We have also seen that both the lower central series and the dimension series $\left\{\delta_{n, k}(G)\right\}$ are both $N$-series. More generally, for every filtration

$$
\varpi_{k}(G)=\mathfrak{a}_{1} \supseteq \mathfrak{a}_{2} \supseteq \mathfrak{a}_{3} \supseteq \cdots
$$

satisfying $\mathfrak{a}_{i} \mathfrak{a}_{j} \subseteq \mathfrak{a}_{i+j}$ for all $i, j \geqslant 1$, the series $\left\{D_{k}\left(\mathfrak{a}_{i}\right)\right\}_{i \geqslant 1}$ is an $N$-series of $G$ by Lemma 2.67.

An importanant example of an $N_{0}$ series is the following. For a group $G$ and a subgroup $H \subseteq G$, define the isolator of $H$ to be $\sqrt{H}=\left\{g \in G: g^{i} \in H\right.$ for some $i \geqslant$ $1\}$. Note that $\sqrt{H}$ is not necessarily a subgroup of $G$, as for example $\sqrt{\{e\}}$ is the set of torsion elements of $G$, which is not always a subgroup. However, if $G$ is nilpotent, the set of torsion elements of $G$ indeed forms a subgroup. Thus, for 
every $n \geqslant 1$, the set $\sqrt{\gamma_{n}(G)}$ is a subgroup of $G$. One then easily verifies that the series $\left\{\sqrt{\gamma_{n}(G)}\right\}_{n \geqslant 1}$ is an $N_{0^{-}}$series for every group $G$.

Every $N$-series

$$
G=G_{1} \supseteq G_{2} \supseteq G_{3} \supseteq \cdots
$$

defines a weight function on $G$ by setting

$$
\operatorname{wt}(g)=\max \left\{n \in \mathbb{N}: g \in G_{n}\right\} \in \mathbb{N} \cup\{\infty\} .
$$

By the defining property of the $N$-series, the weight function satisfies wt $([g, h]) \geqslant$ $\operatorname{wt}(g)+\operatorname{wt}(h)$ for all $g, h \in G$. Also, every $N$-series of a group $G$ induces a descending series $\left\{I_{n}\right\}_{n \geqslant 1}$ of ideals of $k G$ by setting $I_{n}$ to be the ideal generated by all products $\left(g_{1}-1\right) \cdots\left(g_{r}-1\right)$ with $\sum_{i} \operatorname{wt}\left(g_{i}\right) \geqslant n$. Then, as $I_{1}=\varpi_{k}(G)$ and $I_{i} I_{j} \subseteq I_{i+j}$, we have $\varpi_{k}^{n}(G) \subseteq I_{n}$ for every $n \geqslant 1$. A major result was proven by Jennings [23].

Theorem 2.69 (Jennings [23]). If $k$ is a field of characteristic zero, and $G$ is an arbitrary group, then for all $n \geqslant 1$ we have

$$
\delta_{n, k}(G)=\sqrt{\gamma_{n}(G)} .
$$

Proof. We first prove the inclusion $\sqrt{\gamma_{n}(G)} \subseteq \delta_{n, k}(G)$. The inclusion is clear for $n=1$, so let $n>1$ and $g \in \sqrt{\gamma_{n}(G)}$. Then there is $i \geqslant 1$, such that $g^{i} \in \gamma_{n}(G) \subseteq \delta_{n, k}(G)$, and therefore by definition $g^{i}-1 \in \varpi_{k}^{n}(G)$. Then, as

$$
g^{i}-1=\sum_{j=1}^{i}\left(\begin{array}{l}
i \\
j
\end{array}\right)(g-1)^{j}
$$

we conclude that

$$
(g-1)^{n-2}\left(g^{i}-1\right)=\sum_{j=1}^{i}\left(\begin{array}{l}
i \\
j
\end{array}\right)(g-1)^{j+n-2} \in \varpi_{k}^{n}(G),
$$

and as $(g-1)^{j+n-2} \in \varpi_{k}^{n}(G)$ for $j>1$, it follows that $\left(\begin{array}{l}i \\ 1\end{array}\right)(g-1)^{n-1} \in \varpi_{k}^{n}(G)$. Now $i$ is invertible in the field $k$, so $(g-1)^{n-1} \in \varpi_{k}^{n}(G)$.

Now if $n=2$ we are done. Otherwise, we see that $(g-1)^{n-3}\left(g^{i}-1\right) \in \varpi_{k}^{n}(G)$, and as by the above calculation $(g-1)^{n-1} \in \varpi_{k}^{n}(G)$, we conclude that $\left(\begin{array}{l}i \\ 2\end{array}\right)(g-1)^{n-2} \in$ $\varpi_{k}^{n}(G)$, and therefore $(g-1)^{n-2} \in \varpi_{k}^{n}(G)$. Continuing that way, we see that $g-1 \in \varpi_{k}^{n}(G)$, and thus $g \in \delta_{n, k}(G)$ as required.

For the reverse inclusion, first note that it follows from the above that the descending series of ideals induced by the $N_{0}$-series $\left\{\sqrt{\gamma_{n}(G)}\right\}_{n \geqslant 1}$ is precisely the series of powers of $\varpi_{k}(G)$. Also note that it is sufficient to prove the assertion only for finitely generated groups, and that it is equivalent to prove that $\delta_{c+1, k}(G)=\{1\}$ for every group $G$ with $\sqrt{\gamma_{c+1}(G)}=\{1\}$, so all in all we can assume that $G$ is finitely generated, torsion free and nilpotent. Then it can be shown that the lower central series of such a group can be refined to a central series

$$
G=H_{1} \supseteq H_{2} \supseteq \cdots \supseteq H_{r} \supseteq H_{r+1}=\{1\},
$$


of normal subgroups, such that $H_{i} / H_{i+1}$ is infinite cyclic. Then $\gamma_{j}(G)=H_{i_{j}}$ for some index $i_{j}$. Choose then $x_{1}, \ldots, x_{r} \in G$, such that $x_{i} H_{i+1}$ generates $H_{i} / H_{i+1}$ for every $i$. Then every $g \in G$ can be uniquely written as

$$
g=x_{1}^{k_{1}} \cdots x_{r}^{k_{r}}
$$

with $k_{1}, \ldots, k_{r} \in \mathbb{Z}$

For $j \leqslant r$, set $u_{j}=1-x_{j} \in k G$. Fix $M \geqslant 1$. For $d_{j} \in \mathbb{Z}$, set

$$
v_{j}\left(d_{j}\right)= \begin{cases}u_{j}^{d_{j}} & \text { if } d_{j} \geqslant 0 \\ u_{j}^{M} x_{j} & \text { if } d_{j}<0\end{cases}
$$

Then it can be shown that the set

$$
\left\{v_{1}\left(d_{1}\right) \cdots v_{r}\left(d_{r}\right): d_{1}, \ldots, d_{r} \in \mathbb{Z}\right\}
$$

is a $k$-basis of $k G$. Therefore, $\varpi_{k}(G)$ is spanned by all $v_{1}\left(d_{1}\right) \cdots v_{r}\left(d_{r}\right)$ with $\left(d_{1}, \ldots, d_{r}\right) \neq(0, \ldots, 0)$. Now we can define a function $\overline{\mathrm{wt}}$ on all products of the form $v_{1}\left(d_{1}\right) \cdots v_{r}\left(d_{r}\right)$ with $d_{1}, \ldots, d_{r} \in \mathbb{Z}$ by setting

$$
\overline{\mathrm{wt}}\left(v_{1}\left(d_{1}\right) \cdots v_{r}\left(d_{r}\right)\right)= \begin{cases}\sum_{j} d_{j} \mathrm{wt}\left(x_{j}\right) & \text { if } d_{j} \geqslant 0 \text { for all } j, \\ M & \text { otherwise, }\end{cases}
$$

where wt is the weight function with respect to the lower central series. Then this function defines a series $E_{n}$ given by

$$
E_{n}=\left\{\begin{array}{l}
\operatorname{span}\left\{v_{1}\left(d_{1}\right) \cdots v_{r}\left(d_{r}\right): \overline{\mathrm{wt}}\left(v_{1}\left(d_{1}\right) \cdots v_{r}\left(d_{r}\right)\right) \geqslant n\right\} \text { if } n \leqslant M \\
E_{M} \text { else. }
\end{array}\right.
$$

Then it can be shown that $E_{n}=J_{n}$ for all $n \leqslant M$, where $\left\{J_{n}\right\}_{n \geqslant 1}$ is the filtration of $\varpi_{k}(G)$ arising from the series (2.14). Therefore, $D_{k}\left(E_{n}\right)=H_{n}$ for all $n \leqslant M$, and consequently if we choose $M \geqslant r+1$, we get $D_{k}\left(E_{r+1}\right)=\{1\}$. Then, as $\varpi_{k}^{n}(G) \subseteq E_{n}$, we conclude that $\delta_{c+1, k}(G)=\{1\}$ as desired.

Corollary 2.70. For every group $G$ and every $n \geqslant 1$, the $n$-th dimension quotient $\delta_{n}(G) / \gamma_{n}(G)$ is a torsion group.

Proof. Let $g \in \delta_{n}(G)$. Then $g-1 \in \varpi_{\mathbb{Z}}^{n}(G) \subseteq \varpi_{\mathbb{Q}}^{n}(G)$, so $g \in \delta_{n, \mathbb{Q}}(G)$. Therefore, by Theorem 2.69, $g^{i} \in \gamma_{n}(G)$ for some $i \geqslant 1$, as required.

The next step is to understand the dimension subgroups over field of characteristic $p>0$. So fix a prime number $p$. Then, for a given group $G$, define the series of subgroups

$$
G_{n}=\prod_{i p^{j} \geqslant n} \gamma_{i}(G)^{p^{j}}, n \geqslant 1 .
$$

Define another series $\left\{M_{n}(G)\right\}_{n \geqslant 1}$ recursively by setting

$$
M_{1}(G)=G, M_{n}(G)=\left[G, M_{n-1}(G)\right] M_{[n / p]}^{p}(G), n \geqslant 2 .
$$

Here, $\lceil n / p\rceil$ denotes the smallest integer greater or equal to $n / p$. 
Theorem 2.71 (Lazard [26]). Let $k$ be a field of characteristic $p>0$, and $G$ be an arbitrary group. Then, for all $n \geqslant 1$,

$$
G_{n}=M_{n}(G)=\delta_{n, k}(G)
$$

Let us now consider $k=\mathbb{Z}$. Here it was conjectured that $\delta_{n}(G)=\gamma_{n}(G)$ for every group $G$ and every $n \geqslant 1$. If a group $G$ satisfies this, we will say that $G$ has the dimension property.

We have already seen that $\gamma_{n}(G) \subseteq \delta_{n}(G)$ for every $n \geqslant 1$ and every group $G$. Hence the inclusion $\gamma_{n}(G) \hookrightarrow \delta_{n}(G)$ induces a homomorphism of abelian groups

$$
\iota: \gamma_{n}(G) / \gamma_{n+1}(G)=\operatorname{gr}_{n}(G) \rightarrow \delta_{n}(G) / \delta_{n+1}(G)
$$

for every $n \geqslant 1$, and hence a Lie ring homomorphism

$$
\bar{\iota}: \bigoplus_{n \geqslant 1} \gamma_{n}(G) / \gamma_{n+1}(G) \rightarrow \bigoplus_{n \geqslant 1} \delta_{n}(G) / \delta_{n+1}(G)
$$

where the Lie ring structure of both sides is as in Example 2.16. The group $G$ then has the dimension property, if and only if $\bar{\iota}$ is a monomorphism.

Theorem 2.72 (Magnus [29]). Every free group $F$ has the dimension property.

Proof. Let $F$ be a free group on a set $X$. Then the Lie algebra $\operatorname{gr}(F)$ is isomorphic to the free Lie algebra on $X$ (for a detailed proof of that fact see [29] or [17]). Set $\overline{\mathrm{gr}}_{n}(F)=\varpi^{n}(F) / \varpi^{n+1}(F)$ for $n \geqslant 0$, and

$$
\overline{\mathrm{gr}}(F)=\bigoplus_{n \geqslant 0} \overline{\mathrm{gr}}_{n}(F) .
$$

Then $\overline{\operatorname{gr}}(F)$ is an associative algebra with componentwise addition and multiplication defined by $\left(u+\varpi^{n+1}(F)\right)\left(v+\varpi^{m+1}(F)\right)=u v+\varpi^{n+m+1}(F)$ for $u \in \varpi^{n}(F), v \in$ $\varpi^{m}(F)$. Furthermore there is an algebra isomorphism $\mathbb{Z}\langle X\rangle \simeq \overline{\operatorname{gr}}(F)$ induced by the map $x \mapsto(x-1)+\varpi^{2}(F)$ for every $x \in X$. Consider the sequence of maps

$$
\operatorname{gr}(F) \stackrel{\bar{\iota}}{\rightarrow} \bigoplus_{n \geqslant 1} \delta_{n}(F) / \delta_{n+1}(F) \rightarrow \overline{\operatorname{gr}}(F),
$$

where the second map is given by $u \delta_{n+1}(F) \mapsto(u-1)+\varpi^{n+1}(F)$. Now the composition of the two maps is precisely the embedding of the free Lie algebra into its universal enveloping algebra, the free associative algebra, and therefore injective by Theorem 2.29. Thus, $\bar{\imath}$ is injective, as required.

The procedure to investigate dimension subgroups of an arbitrary group $G$ is now the following. Write $G \simeq F / R$ with a free group $F$ and a normal subgroup $R \subseteq F$. Set $\mathfrak{f}=\varpi(F)$, and denote by $\mathfrak{r}$ the kernel of the canonical projection $\mathbb{Z} F \rightarrow \mathbb{Z} G$, i.e. $\mathfrak{r}=\mathbb{Z} F(R-1)$. 
Lemma 2.73. With the above notation, we have

$$
\begin{aligned}
\gamma_{n}(G) & \simeq \gamma_{n}(F) R / R, \\
\varpi^{n}(G) & \simeq\left(1+\mathfrak{f}^{n}+\mathfrak{r}\right) / \mathfrak{r}, \\
\delta_{n}(F) & \simeq\left(F \cap\left(1+\mathfrak{f}^{n}+\mathfrak{r}\right)\right) / R
\end{aligned}
$$

for every $n \geqslant 1$.

Proof. The proof is exactly the same as the proof of Lemma 3.7.

As for a free group $F$ we have $\gamma_{n}(F)=\delta_{n}(F)$ for all $n \geqslant 1$, the strategy to investigate dimension subgroups is as follows. Let $G$ be a group, then write $G=$ $F / R$ for a free group $F$ and a normal subgroup $R$ of $F$. Then, with the notation above, $\gamma_{n}(G) \simeq \gamma_{n}(F) R / R$, and $\varpi^{n}(G) \simeq\left(\mathfrak{f}^{n}+\mathfrak{r}\right) / \mathfrak{r}$ for all $n \geqslant 1$. Then the $n$-th dimension quotient is isomorphic to $\left(F \cap\left(1+\mathfrak{f}^{n}+\mathfrak{r}\right)\right) / \gamma_{n}(F) R$. Thus a group $G$ has the dimension property if and only if $F \cap\left(1+\mathfrak{f}^{n}+\mathfrak{r}\right)=\gamma_{n}(F) R$ for every presentation $G \simeq F / R$.

Proposition 2.74 (Gupta [17, IV. Proposition 3.1]). A group G given by a presentation $G \simeq F / R$ has the dimension property for a given $n \geqslant 1$, if and only if

$$
F \cap\left(1+\mathfrak{f}^{n}+\mathfrak{f r}\right) \subseteq \gamma_{n}(F) R .
$$

Proof. It is clear that if $\delta_{n}(G)=\gamma_{n}(G)$ for a given $n \geqslant 1$, we have $F \cap\left(1+\mathfrak{f}^{n}+\mathfrak{f r}\right) \subseteq$ $F \cap\left(1+\mathfrak{f}^{n}+\mathfrak{r}\right)=\gamma_{n}(F) R$.

Suppose now that $F \cap\left(1+\mathfrak{f}^{n}+\mathfrak{f r}\right) \subseteq \gamma_{n}(F) R$ holds, and let $u \in F \cap\left(1+\mathfrak{f}^{n}+\mathfrak{r}\right)$. Then $u-1 \in \mathfrak{f}^{n}+\mathfrak{r}$, so $u-1=u_{1}+u_{2}$ with $u_{1} \in \mathfrak{f}^{n}$ and $u_{2} \in \mathfrak{r}=\mathbb{Z} F(R-1)$. Then $u_{2}=\sum_{j} v_{j}\left(r_{j}-1\right)$ for some $v_{j} \in \mathbb{Z} F$. Write $v_{j}=\varepsilon\left(v_{j}\right)+v_{j}^{\prime}$ with $v_{j}^{\prime} \in \mathfrak{f}$. Then, modulo $\mathfrak{f r}$, we can write $u_{2} \equiv \sum_{j} n_{j}\left(r_{j}-1\right)$, where $n_{j}=\varepsilon\left(v_{j}\right) \in \mathbb{Z}$. Then, again modulo $\mathfrak{f} \mathfrak{r} \subseteq \mathfrak{f}^{2}$, we have $u_{2} \equiv \prod_{j} r_{j}^{n_{j}}-1=: r-1$.

Therefore, as $u r^{-1}-1 \equiv(u-1)-(r-1) \bmod \mathfrak{f}^{n}+\mathfrak{f r}$, we conclude $u r^{-1} \in$ $F \cap\left(1+\mathfrak{f}^{n}+\mathfrak{f r}\right) \subseteq \gamma_{n}(F) R$, and as $r \in R \subseteq \gamma_{n}(F) R$, it follows that $u \in \gamma_{n}(F) R$ as required.

Thus, the identification of $F \cap\left(1+\mathfrak{f}^{n}+\mathfrak{f r}\right)$ is crucial. By Lemma 2.67, we have $F \cap\left(1+\mathfrak{f}^{n}+\mathfrak{f r}\right) \supseteq\left(F \cap\left(1+\mathfrak{f}^{n}\right)\right)(F \cap(1+\mathfrak{f} \mathfrak{r}))=\gamma_{n}(F)(F \cap(1+\mathfrak{f r}))$. The latter group was identified as $R^{\prime}=[R, R]$ by Schumann [41] already in 1937. We include a more general result due to Bergman and Dicks here.

Theorem 2.75 (Bergman, Dicks [4]). Let $N, M \subseteq G$ be normal subgroups of a group $G$, and set $\mathfrak{n}=\mathbb{Z} G(N-1)$ and $\mathfrak{m}=\mathbb{Z} G(M-1)$. Then

$$
G \cap(1+\mathfrak{m m})=[N \cap M, N \cap M]
$$

Back to the original problem, it was conjectured that $\gamma_{n}(G)=\delta_{n}(G)$ holds for all groups $G$ and all $n \geqslant 1$. However, the following counter example has been given by Rips in [39]. 
Example 2.76. Let $G$ be the group generated by $a_{0}, a_{1}, a_{2}, a_{3}, b_{1}, b_{2}, b_{3}$ and $c$ with defining relations

$$
\begin{aligned}
& b_{1}^{64}=b_{2}^{16}=b_{3}^{4}=c^{256}=1, \\
& {\left[b_{i}, b_{j}\right]=\left[b_{i}, c\right]=\left[a_{i}, c\right]=1 \text { for all } i, j \leqslant 3,} \\
& a_{0}^{64}=b_{1}^{32}, a_{1}^{64}=b_{2}^{-4} b_{3}^{-2}, a_{2}^{16}=b_{1}^{4} b_{3}^{-1}, a_{3}^{4}=b_{1}^{2} b_{2}, \\
& {\left[a_{1}, a_{0}\right]=b_{1} c^{2},\left[a_{2}, a_{0}\right]=b_{2} c^{8},\left[a_{3}, a_{0}\right]=b_{3} c^{32},} \\
& {\left[a_{2}, a_{1}\right]=c,\left[a_{3}, a_{1}\right]=c^{2},\left[a_{3}, a_{2}\right]=c^{4},} \\
& {\left[b_{1}, a_{1}\right]=c^{4},\left[b_{2}, a_{2}\right]=c^{16},\left[b_{3}, a_{3}\right]=c^{64},} \\
& {\left[b_{i}, a_{j}\right]=1 \text { if } i \neq j .}
\end{aligned}
$$

Then $\gamma_{4}(G)=\{1\}$, but

$$
1 \neq c^{128}=\left[a_{1}, a_{2}\right]^{128}\left[a_{1}, a_{3}\right]^{64}\left[a_{2}, a_{3}\right]^{32} \in \delta_{4}(G) .
$$

This has been further generalized by Gupta in [19], who gave for every $n \geqslant 4$ an example of a group $G(n)$ with $\delta_{n}(G(n)) \neq \gamma_{n}(G(n))$.

Example 2.77. Let $n \geqslant 4$, and let $F$ be the free group freely generated by $r, a, b, c$. Set $x_{0}=y_{0}=z_{0}=r$, and $x_{i}=\left[x_{i-1}, a\right], y_{i}=\left[y_{i-1}, b\right], z_{i}=\left[z_{i-1}, c\right]$ for $i \geqslant 1$. Let $G(n)$ be the quotient of $F$ by the follwing relations.

$$
\begin{aligned}
& r^{2^{2 n-1}}=x_{n-1}=y_{n-1}=z_{n-1}=1, \\
& a^{2^{n+2}}=y_{n-3}^{4} z_{n-3}^{2}, b^{2^{n}}=x_{n-3}^{-4} z_{n-3}, c^{2^{n-2}}=x_{n-3}^{-2} y_{n-3}^{-1}, \\
& z_{n-2}=y_{n-2}^{4}, y_{n-2}=x_{n-2}^{4}, \\
& {[a, b, g]=[b, c, g]=[a, c, g]=1 \text { for all } g \in F,} \\
& {\left[x_{i}, b\right]=\left[x_{i}, c\right]=\left[y_{i}, a\right]=\left[y_{i}, c\right]=\left[z_{i}, a\right]=\left[z_{i} \cdot b\right]=1 \text { for all } i \geqslant 1,} \\
& {\left[x_{i}, x_{j}\right]=\left[x_{i}, y_{j}\right]=\left[x_{i}, z_{j}\right]=\left[y_{i}, y_{j}\right]=\left[y_{i}, z_{j}\right]=\left[z_{i}, z_{j}\right]=1 \text { for all } i, j \geqslant 0 .}
\end{aligned}
$$

Then $G(n)$ is a metabelian group with $\gamma_{n}(G(n))=\{1\}$, and

$$
1 \neq g=[a, b]^{2^{2 n-1}}[a, c]^{2^{2 n-2}}[b, c]^{2^{2 n-3}} \in \delta_{n}(G(n)) .
$$

In all the examples above, we have $g^{2}=1$.

By Corollary 2.70 $\delta_{n}(G) / \gamma_{n}(G)$ is a torsion group for every group $G$ and every $n \geqslant 1$. It was shown by Sjogren in [45] (see also [8] for another proof), that the exponent of the $n$-th dimension quotient is bounded by a constant depending only on $n$.

Theorem 2.78 (Sjogren [45]). Define $b_{m}=\operatorname{lcm}(1, \ldots, m)$, and set

$$
c_{n}=\prod_{i=1}^{n-2} b_{i}^{\left(\begin{array}{c}
n-2 \\
i
\end{array}\right)} .
$$

Then, for every group $G$, the exponent of $\delta_{n}(G) / \gamma_{n}(G)$ divides $c_{n}$, in other words $\delta_{n}(G)^{c_{n}} \subseteq \gamma_{n}(G)$. 
As all prime divisors of $c_{n}$ are at most $n-2$, we easily conclude the following corollary.

Corollary 2.79. Let $G$ be a p-group. Then $\delta_{n}(G)=\gamma_{n}(G)$ for all $n \leqslant p+1$.

We give a very brief sketch of Sjogren's proof here, as it motivates the use of spectral sequences, which we will use later, when we analyze the Lie dimension subring problem.

Write a given group $G$ as presentation $G \simeq F / R$ with a free group $F$ and a normal subgroup $R \subseteq F$. Let $F$ be freely generated by a set $\left\{x_{i}: i \in I\right\}$, and $R$ be generated by $\left\{r_{j}: j \in J\right\}$. Then let $F * R$ be the free product of $F$ and $R$, i.e. the free group generated by the disjoint union $\left\{x_{i}: i \in I\right\} \sqcup\left\{r_{j}: j \in J\right\}$. Then there are two group homomorphisms

$$
\alpha: \begin{cases}F * R & \rightarrow F \\ x_{i} & \mapsto x_{i} \\ r_{j} & \mapsto 1\end{cases}
$$

and

$$
\beta: \begin{cases}F * R & \rightarrow F \\ x_{i} & \mapsto x_{i} \\ r_{j} & \mapsto r_{j} .\end{cases}
$$

Then, setting $K=\operatorname{ker}(\alpha)$, we note that $K$ is the normal subgroup of $F * R$ generated by $\left\{r_{j}: j \in J\right\}$. Therefore, $\beta(K)=R$, thus $G \simeq F / \beta(K)$. In other words, the "chain complex" $K \stackrel{\beta}{\rightarrow} F$ has 0-th homology isomorphic to $G$.

For $n \geqslant 1$ set $K_{n}=K \cap \gamma_{n}(F * R)$. Then this chain complex can be filtered as follows.

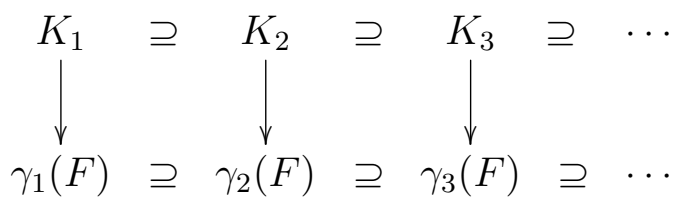

Here, the vertical maps are restrictions of $\beta$. Now by the construction of MacLane [28. Chapter XI, Theorem 3.1], this leads to a spectral sequence $E$, where $E_{p, q}^{r}=0$ for $p+q \neq 0,1$, and $E_{p, q}^{r}=0$ for $p \geqslant 0$. The differential $d_{p, q}^{r}$ on the $r$-th page of the spectral sequence has bidegree $(-r, r-1)$, i.e. $d_{p, q}^{r}: E_{p, q}^{r} \rightarrow E_{p-r, q+r-1}^{r}$. It follows that $E_{-r, r}^{r}=E_{-r, r}^{r+1}=\cdots=E_{-r, r}^{\infty}$. Also, by [28, Chapter XI, Theorem 3.1], $E_{-r, r}^{\infty} \simeq \gamma_{r}(G) / \gamma_{r+1}(G)$.

Similarly, the maps $\alpha$ and $\beta$ above extend to maps $\alpha^{*}, \beta^{*}: \mathbb{Z}(F * R) \rightarrow \mathbb{Z} F$, and we set $\tilde{K}=\operatorname{ker}\left(\alpha^{*}\right)$. Then setting $\tilde{K}_{n}=\tilde{K} \cap \varpi^{n}(F * R)$, we obtain another filtered chain complex

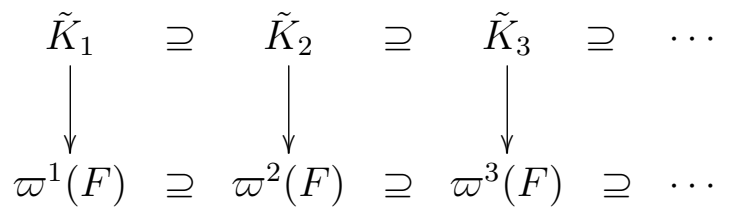


and another spectral sequence $\tilde{E}$ with $\tilde{E}_{-r, r}^{r}=\tilde{E}_{-r, r}^{r+1}=\cdots=\tilde{E}_{-r, r}^{\infty} \simeq \varpi^{r}(G) / \varpi^{r+1}(G)$. Now the map $\iota: g \mapsto g-1$ induces a map $\bar{\iota}: E \rightarrow \tilde{E}$. The kernel of the restriction of $\bar{\iota}$ to $E_{-r, r}^{\infty}$ will then determine the $r$-th dimension subgroup. Analyzing the kernels page by page leads then to the result.

The sequence $\left\{c_{n}\right\}_{n \geqslant 1}$ grows very fast, e.g. $c_{4}=2, c_{5}=48, c_{6}=995328, \ldots$ For $n=5$, the bound on the exponent has been bettered by Tahara.

Theorem 2.80 (Tahara [48]). For every group $G$,

$$
\delta_{5}(G)^{6} \subseteq \gamma_{5}(G)
$$

A similar approach to the dimension subgroup problem was made by Grünenfelder 14. For a given group $G$, he defined a free simplicial group $\mathbf{F}$ with $\pi_{0}(\mathbf{F}) \simeq G$ and $\pi_{n}(\mathbf{F})=\{1\}$ for all $n \geqslant 1$. If $G \simeq F / R$ with a free group $F$, a possible choice for such a resolution is $F_{0}=F$ and $F_{1}=F * R$, as in Sjogren's proof. The simplicial maps $d_{0}, d_{1}: F_{1} \rightarrow F_{0}$ are then $\alpha$ and $\beta$ from Sjogren's proof. However, there are probably also higher non-trivial free groups $F_{2}, F_{3}, \ldots$ extending the diagram to a simplicial resolution. The techniques are similar to the ones used later on in this thesis for the Lie dimension subalgebra problem, and will be explained there in further detail.

As for general groups, computations turn out to be quite long and hard. If one assumes further that the group $G$ is metabelian (i.e. $G^{\prime \prime}=\left[G^{\prime}, G^{\prime}\right]=\{1\}$ ), better results are known.

To obtain such results, it is useful to give a more specific way of a group presentation.

Theorem 2.81 ([31, Theorem 3.5]). Let $G$ be a finitely generated group. Then there exists a free group $F$ on $X=\left\{X_{1}, \ldots, X_{m}\right\}$ and a normal subgroup $R \subseteq F$, such $G \simeq F / R$ and $R$ is given by

$$
R=\left\langle X_{1}^{e_{1}} \xi_{1}, \ldots, X_{m}^{e_{m}} \xi_{m}, \xi_{m+1}, \ldots\right\rangle
$$

with $\xi_{i} \in F^{\prime}$ and integers $e_{i}$ satisfying $e_{i} \mid e_{i+1}$ for every $i<m$. Such a presentation will be called a pre-abelian presentation.

If a group $G$ is given by a pre-abelian presentation, then its abelianization $G / \gamma_{2}(G)$ is given by

$$
G / \gamma_{2}(G) \simeq \mathbb{Z} /\left(e_{1}\right) \oplus \cdots \oplus \mathbb{Z} /\left(e_{m}\right)
$$

Set $S=R F^{\prime}=\left\langle X_{1}^{e_{1}}, \ldots, X_{m}^{e_{m}}, F^{\prime}\right\rangle$. Then $G / \gamma_{2}(G) \simeq F / S$. If $G$ is a metabelian group, then by definition $G^{\prime \prime}=\{1\}$. Therefore, for a presentation $G \simeq F / R$ we will have $F^{\prime \prime} \subseteq R$, and thus $S^{\prime} \subseteq R \subseteq S$. Also, we can regard $F^{\prime} / F^{\prime \prime}$ as $\mathbb{Z} F$-module via conjugation, i.e.

$$
\left(w F^{\prime \prime}\right)^{u}=u^{-1} w u F^{\prime \prime}
$$


for all $w \in F^{\prime}, u \in F$, extended linearly. Then, for all $x, y, z \in F$ and $n \geqslant 1$, we have modulo $F^{\prime \prime}$

$$
\begin{aligned}
{[[x, y], z][[y, z], x][[z, x], y] } & \equiv 1, \\
{\left[x^{n}, y\right] } & \equiv[x, y]^{1+x+\cdots+x^{n-1}}, \\
{[x, y]^{z-1} } & \equiv[x, y, z] .
\end{aligned}
$$

Then set

$$
t\left(X_{i}, e_{i}\right)=\sum_{k=0}^{e_{i}-1} X_{i}^{k}
$$

for $1 \leqslant i \leqslant m$. Note that $\left(X_{i}-1\right) t\left(X_{i}, e_{i}\right) \in \mathfrak{s}$ for all $i$. Then, due to Gupta, we have:

Theorem 2.82 (Gupta [16]). For all $n \geqslant 1$, modulo $\left[F^{\prime}, S\right] \gamma_{n}(F)$ the subgroup $F \cap\left(1+\mathfrak{f}^{n}+\mathfrak{f} \mathfrak{s}\right)$ of $F$ is generated by all elements of the form

$$
\left[X_{i}, X_{j}\right]^{t\left(X_{i}, e_{i}\right) a_{i j}}, m \geqslant i>j \geqslant 1,
$$

with $a_{i j} \in \mathbb{Z} F$ depending only on $X_{1}, \ldots, X_{j}$ and $t\left(X_{i}, e_{i}\right) a_{i j} \in t\left(X_{j}, e_{j}\right) \mathbb{Z} F+\mathfrak{s}+\mathfrak{f}^{n}$.

From this description and the Artin-Rees-Theorem, one can conclude the following result on dimension subgroups of metabelian groups.

Theorem 2.83 (Gupta, Hales and Passi [15]). Let $G$ be a finitely generated metabelian group. Then there is $n_{0}\left(G / G^{\prime}\right) \geqslant 1$ depending only on the abelianization of $G$, such that $\delta_{n}(G)=\gamma_{n}(G)$ for all $n \geqslant n_{0}\left(G / G^{\prime}\right)$.

Proof. Let $G$ be a finitely generated metabelian group, and $G \simeq F / R$ a pre-abelian presentation of $G$ with a free group $F$ on a finite set $X=\left\{X_{1}, \ldots, X_{m}\right\}$. As above, set $S=R \gamma_{2}(F)=\left\langle X_{1}^{e_{1}}, \ldots, X_{m}^{e_{m}}, F^{\prime}\right\rangle$ with $e_{i} \mid e_{i+1}$, and $\mathfrak{s}=\mathbb{Z} F(S-1)$. We will show that there exists $n_{0}\left(G / G^{\prime}\right) \geqslant 1$, such that $F \cap\left(1+\mathfrak{f}^{n}+\mathfrak{f} \mathfrak{s}\right)=S^{\prime} \gamma_{n}(F)$ for all $n \geqslant n_{0}(G)$. As $\mathfrak{r} \subseteq \mathfrak{s}$ and $S^{\prime} \subseteq R$, by Proposition 2.74, this implies the Theorem. Note that $F / S$ is abelian, and thus $\mathbb{Z}(F / S) \simeq \mathbb{Z} F / \mathfrak{s}$ is a commutative ring. As $F$ is finitely generated, $\mathbb{Z} F / \mathfrak{s}$ is also Noetherian. Therefore, by the Artin-ReesTheorem (see e.g. [25, p. 429]), for any two ideals $U, V$ of $\mathbb{Z} F / \mathfrak{s}$, there exists $n_{0}$ such that $U \cap V^{n}=\left(U \cap V^{n_{0}}\right) V^{n-n_{0}}$ for all $n \geqslant n_{0}$.

Now, for $1 \leqslant i \leqslant m$, let $U_{i}$ be the ideal generated by $t\left(X_{i}, e_{i}\right)$, and set $V=\mathfrak{f} / \mathfrak{s}$. Then, if $a_{i j} \in \mathbb{Z} F$ are given such that $t\left(X_{i}, e_{i}\right) a_{i j} \in t\left(X_{j}, e_{j}\right) \mathbb{Z} F+\mathfrak{s}+\mathfrak{f}^{n}$, then $\left(X_{j}-1\right) t\left(X_{i}, e_{i}\right) a_{i j} \in \mathfrak{s}+\mathfrak{f}^{n+1}$, so in $\mathbb{Z} F / \mathfrak{s}$ we get $\left(X_{j}-1\right) t\left(X_{i}, e_{i}\right) a_{i j} \in U_{i} \cap V^{n+1}=$ $\left(U_{i} \cap V^{n_{0}}\right) V^{n+1-n_{0}}$ for some $n_{0} \in \mathbb{N}$ and all $n+1 \geqslant n_{0}$. Therefore, modulo $\mathfrak{s}$ we can write

$$
\left(X_{j}-1\right) t\left(X_{i}, e_{i}\right) a_{i j} \equiv \sum_{k} t\left(X_{i}, e_{i}\right) \alpha_{k} \beta_{k}
$$

where $\beta_{k} \in \mathfrak{f}^{n+1-n_{0}}$ and the $\alpha_{k}$ are such that $t\left(X_{i}, e_{i}\right) \alpha_{k} \in \mathfrak{f}^{n_{0}}+\mathfrak{s}$. As $\beta \in \mathfrak{f}^{n+1-n_{0}}$, there are $\xi_{k, \ell} \in \mathfrak{f}^{n-n_{0}}$, such that

$$
\beta_{k}=\sum_{\ell=1}^{m}\left(X_{\ell}-1\right) \xi_{k, \ell} .
$$


Then one can show that there are $f_{i j} \in\left[F^{\prime}, S\right] \gamma_{n+2}(F)$ with

$$
\left[X_{i}, X_{j}\right]^{t\left(X_{i}, e_{i}\right) a_{i j}}-1 \equiv f_{i j}-1 \quad \bmod \mathfrak{f} \mathfrak{s}
$$

and therefore

$$
\left[X_{i}, X_{j}\right]^{t\left(X_{i}, e_{i}\right) a_{i j}} f_{i j}^{-1} \in F \cap(1+\mathfrak{f} \mathfrak{s})=S^{\prime}
$$

by Schumann [41, and so $\left[X_{i}, X_{j}\right]^{t\left(X_{i}, e_{i}\right) a_{i j}} \in S^{\prime} \gamma_{n+2}(F)$. As by Theorem 2.82 these elements generate $F \cap\left(1+\mathfrak{f}^{n+1}+\mathfrak{f} \mathfrak{s}\right) /\left[F^{\prime}, S\right] \gamma_{n+2}(F)$, the theorem follows.

Also, if $G$ is metabelian, the exponents from Sjogren's Theorem could be significantly bettered, and turn out to be a power of 2 .

Theorem 2.84 (Gupta [20]). Let $G$ be a finitely generated metabelian group. Then the exponent of $\delta_{n}(G) / \gamma_{n}(G)$ divides $2^{\left(\begin{array}{c}n-2 \\ 2\end{array}\right)}$.

Thus, it follows that for every metabelian group of odd order, the dimension series and lower central series coincide.

Also, the group structure of the quotient $\delta_{n}(G) / \gamma_{n}(G)$ has been investigated for metabelian groups.

Theorem 2.85 (Gupta [18]). If $G$ is a metabelian group, then, for all $n \geqslant 1$ we have

$$
\left[\delta_{n}(G), G\right]=\gamma_{n+1}(G) .
$$

In particular, the quotient group $\delta_{n}(G) / \gamma_{n}(G)$ is abelian for all $n \geqslant 1$.

Though a lot has been found out about dimension subgroups, there are still some open problems about them given in [17] and [33, which we name here.

Problem 2.86. Does Theorem 2.83 hold for general finitely generated groups as well?

Problem 2.87. By Theorem 2.85, all dimension quotients are abelian groups, if $G$ is metabelian. Does that hold for general groups as well?

Problem 2.88. Is there a group $G$, such that $\gamma_{4}(G)=\{1\}$, but $\delta_{5}(G) \neq\{1\}$ ? Or, more generally, given $n \geqslant 4$, is there a number $m(n) \in \mathbb{N}$, such that $\delta_{m(n)}(G) \subseteq$ $\gamma_{n}(G)$ for all groups $G$ ?

Problem 2.89. Is $\left[\delta_{5}(G), G, G\right]=\gamma_{7}(G)$ for all groups $G$ ? 


\section{The Lie Dimension Problem}

In the world of Lie algebras, a problem similar to the Dimension Subgroup Problem can be stated. Take a Lie algebra $L$ over a commutative ring $k$, and assume for simplicity that $\iota: L \rightarrow \mathcal{U} L$ is injective. Then every Lie ideal $A \subseteq L$ defines a two-sided ideal $\mathcal{U} L \cdot A$ of $\mathcal{U} L$, so we obtain a map

$$
I: \begin{cases}\{\text { Lie ideals of } L\} & \rightarrow\{\text { ideals of } \mathcal{U} L\}, \\ A & \mapsto \mathcal{U} L \cdot A .\end{cases}
$$

On the other hand, for every ideal $\mathfrak{a} \subseteq \mathcal{U} L$, the set $L \cap \mathfrak{a}$ is an ideal in $L$. Thus we get a map in the other direction

$$
D: \begin{cases}\{\text { ideals of } \mathcal{U} L\} & \rightarrow\{\text { Lie ideals of } L\}, \\ \mathfrak{a} & \mapsto L \cap \mathfrak{a} .\end{cases}
$$

Again the question is how these maps are connected.

Proposition 3.1. With the notation as above, we have

$$
D(I(A))=A
$$

for every ideal $A \subseteq L$.

Proof. First observe that $A \subseteq I(A)$ (as $1 \in \mathcal{U} L)$, and therefore $A \subseteq D(I(A)$ ).

For the reverse inclusion, recall that by Theorem $2.23 I(A)$ is the kernel of the map $\pi^{*}: \mathcal{U} L \rightarrow \mathcal{U}(L / A)$ induced by the canonical projection $\pi: L \rightarrow L / A$. Thus, if $a \in D(I(A))$, then $a \in L$ and $\pi(a)=\pi^{*}(a)=0$, and therefore $a \in A$ as desired.

Again the reverse composition is not the identity, as the trivial example $\mathfrak{a}=\mathcal{U} L \cdot L$, $\mathfrak{b}=\mathcal{U} L$ with $D(\mathfrak{a})=D(\mathfrak{b})=L$ but $\mathfrak{a} \neq \mathfrak{b}$ shows.

Let us look at some examples first before examining the problem further. Let first $L$ be a finite dimensional abelian Lie algebra over a field $k$, in other words just a $k$ vector space. Let $X_{1}, \ldots, X_{n}$ be a basis of $L$. Then $\mathcal{U} L \simeq \operatorname{Sym}(L) \simeq k\left[X_{1}, \ldots, X_{n}\right]$ (here $\operatorname{Sym}(L)$ denotes the symmetric algebra of $L$ defined in Example 2.10). Now the Lie ideals of $L$ are just subvectorspaces $A$, spanned by some $Y_{1}, \ldots, Y_{m} \in L$ with $m \leqslant n$. Then we can write $Y_{j}=\sum_{i} a_{i j} X_{i}$ for some $a_{i j} \in k$. Then the ideal $\operatorname{Sym}(L) \cdot A$ is the ideal in the polynomial ring generated by the $Y_{j}=\sum_{i} a_{i j} X_{i}$. Thus the only ideals generated by a subalgebra are the homogeneous ideals of $\operatorname{Sym}(L)$ generated in degree 1 , but there are obviously many more ideals in that algebra.

Take for example $n=1$, so there are just the two trivial ideals of $L$, but infinitely many ideals in $k[X]$. Then one easily sees that $D(\langle X\rangle)=L$, while $D(\mathfrak{a})=0$ for every other ideal $\mathfrak{a} \subseteq \mathcal{U} L$.

We now want to look at an analogue of the dimension subgroup problem in Lie algebras. To do this, first of all we need to define the augmentation ideal in this 
context. By Theorem 2.23, the map $L \rightarrow 0$ extends to a surjective homomorphism of associative algebras $\varepsilon: \mathcal{U} L \rightarrow \mathcal{U} 0 \simeq k$. Then the augmentation ideal of $\mathcal{U} L$ is defined as $\varpi(L)=\operatorname{ker}(\varepsilon)$. As the map $\iota: L \rightarrow \mathcal{U} L$ is an embedding, we can identify $L$ with its image in $\mathcal{U} L$. We then define the $n$-th Lie dimension subring as

$$
\delta_{n}(L)=L \cap \varpi^{n}(L) .
$$

Let $\left\{\gamma_{n}(L)\right\}_{n \geqslant 1}$ be the lower central series of $L$ as defined in 2.20. Then in $\mathcal{U} L$ we have $[x, y]=x y-y x$ for all $x, y \in L$, so by induction on $n$ one verifies that $\gamma_{n}(L) \subseteq \delta_{n}(L)$ for all $n \geqslant 1$. One can ask now, similar to the dimension subgroup problem, whether these two ideals of $L$ are equal.

First of all, it is convenient to answer the question when $k$ is a field. This has been done by Riley [38], who proved equality even for Lie superalgebras, a slight generalization of Lie algebras. We will give his proof only for Lie algebras here.

Theorem 3.2 (Riley [38). Let $k$ be a field of arbitrary characteristic. Then

$$
\delta_{n}(L)=\gamma_{n}(L)
$$

for all $n \geqslant 1$.

Proof. It is equivalent to prove $\delta_{n}(L)=0$ for every Lie algebra $L$ with $\gamma_{n}(L)=0$. So let $L$ be a Lie algebra with $\gamma_{n}(L)=0$. Choose a basis $\mathcal{B}_{n-1}$ of $\gamma_{n-1}(L)$, extend it to a basis $\mathcal{B}_{n-2}$ of $\gamma_{n-2}(L)$, and so on, until we have a basis $\mathcal{B}:=\mathcal{B}_{1}$ of $L$.

For $x \in L$, let $\operatorname{wt}(x)=\max \left\{m \in \mathbb{N}: x \in \gamma_{m}(L)\right\}$, so that $\operatorname{wt}(b)=m$ for $b \in$ $\mathcal{B}_{m} \backslash \mathcal{B}_{m+1}$. We claim that

$$
\varpi^{m}(L)=\operatorname{span}\left\{x_{1} \cdots x_{t}: x_{i} \in L, \sum_{i=1}^{t} \mathrm{wt}\left(x_{i}\right) \geqslant m\right\}=: E_{m} .
$$

It is clear that $E_{m}$ is contained in $\varpi^{m}(L)$. The converse inclusion is true for $m=1$. Thus, $\varpi^{m}(L)=E_{1}^{m} \subseteq E_{m}$, proving Equation (3.1).

Fix a total order on $\mathcal{B}$, and let $\operatorname{PBW}(\mathcal{B})$ be the basis of $\mathcal{U} L$ obtained from $\mathcal{B}$ according to Theorem 2.29. We claim that the subset of $\mathrm{PBW}(\mathcal{B})$ consisting of all $b_{1}^{\alpha_{1}} \cdots b_{t}^{\alpha_{t}}$ with $b_{i} \in \mathcal{B}, b_{1}<\cdots<b_{t}$ and $\sum_{i} \alpha_{i} \operatorname{wt}\left(b_{i}\right) \geqslant m$ is a basis of $E_{m}$.

Indeed, set $E_{m, \ell}=\operatorname{span}\left\{x_{1} \cdots x_{t}: x_{i} \in L, \sum_{i} \operatorname{wt}\left(x_{i}\right) \geqslant m, t \leqslant \ell\right\}$. We will prove by induction on $\ell$ that $E_{m, \ell}$ is spanned by all $b_{1}^{\alpha_{1}} \cdots b_{t}^{\alpha_{t}}$ with $b_{i} \in \mathcal{B}, b_{1}<\cdots<b_{t}$, $\sum_{i} \alpha_{i} \operatorname{wt}\left(b_{i}\right) \geqslant m$ and $\sum_{i} \alpha_{i} \leqslant \ell$. For $\ell=1$ observe that $E_{m, 1}=\gamma_{m}(L)=\operatorname{span}\left(\mathcal{B}_{m}\right)$ by construction of $\mathcal{B}$.

Now assume the statement holds for all $j<\ell$. Consider $x_{1} \cdots x_{\ell} \in E_{m, \ell}$ with $x_{i} \in L$ and $\sum_{i} \operatorname{wt}\left(x_{i}\right) \geqslant m$. Then $x_{1} \cdots x_{\ell-1} \in E_{m-\operatorname{wt}\left(x_{\ell}\right), \ell-1}$, so by the induction hypothesis it can be written as linear combination of $b_{1}^{\alpha_{1}} \cdots b_{t}^{\alpha_{t}}$ with $\sum_{i} \alpha_{i} \operatorname{wt}\left(b_{i}\right) \geqslant$ $m-\operatorname{wt}\left(x_{\ell}\right)$ and $\sum_{i} \alpha_{i} \leqslant \ell-1$. Also, as $x_{\ell} \in L$, it can be written as a linear combination of elements $b \in \mathcal{B}_{\mathrm{wt}\left(x_{\ell}\right)}$. Thus, $x_{1} \cdots x_{\ell}$ is a linear combination of elements of the form $b_{1}^{\alpha_{1}} \cdots b_{t}^{\alpha_{t}} b$, where $b_{i}, \alpha_{i}$ and $b$ have the properties given above. 
Now, if $b_{t} \leqslant b$, the monomial $b_{1}^{\alpha_{1}} \cdots b_{t}^{\alpha_{t}} b$ is of the desired form. If not, use the equation

$$
\begin{array}{r}
b_{1}^{\alpha_{1}} \cdots b_{t}^{\alpha_{t}} b=b_{1}^{\alpha_{1}} b_{t-1}^{\alpha_{t-1}} b b_{t}^{\alpha_{t}}+b_{1}^{\alpha_{1}} \cdots b_{t-1}^{\alpha_{t-1}}\left[b_{t}, b\right] b_{t}^{\alpha_{t}-1} \\
+\cdots+b_{1}^{\alpha_{1}} \cdots b_{t-1}^{\alpha_{t-1}} b_{t}^{\alpha_{t}-1}\left[b_{t}, b\right] .
\end{array}
$$

As $\left[b_{t}, b\right] \in L$, all summands except the first one in the equation above are in $E_{m, \ell-1}$, and thus by induction hypothesis can be written as linear combination of monomials in the desired form. Now if $b_{t-1} \leqslant b$, we are done. Otherwise, continue as above until the summand $b_{1}^{\alpha_{1}} \cdots b_{i}^{\alpha_{i}} b b_{i+1}^{\alpha_{i+1}} \cdots b_{t}^{\alpha_{t}}$ with $b_{i} \leqslant b<b_{i+1}$ arises. All the other summands are in $E_{m, \ell-1}$, so applying the induction hypothesis verifies the claim.

Now, let $x \in \delta_{n}(L)=L \cap \varpi^{n}(L)=L \cap E_{n}$. Then as $x \in L, x$ can be written as a linear combination of elements of $\mathcal{B}$, and as $x \in E_{n}$, all elements $b$ in this linear combination have to satisfy $\operatorname{wt}(b) \geqslant n$, which is not possible as $\gamma_{n}(L)=0$. Thus $x=0$, and $\delta_{n}(L)=\gamma_{n}(L)$.

For $k=\mathbb{Z}$, Bartholdi and Passi started investigating the Lie dimension subrings in 3 .

\subsection{Some first results}

First of all, one may want to look at the problem for a free Lie ring. Here, as in the dimension subgroup problem, both series coincide.

Theorem 3.3 (Bartholdi, Passi [3, Theorem 4.1]). Let $F$ be a free Lie ring with free generating set $X$. Then $\gamma_{n}(F)=\delta_{n}(F)$ for every $n \in \mathbb{N}$.

Proof. By Theorem 2.31, for a free Lie ring $F$ freely generated by $X$, its universal enveloping algebra $\mathcal{U} F$ is isomorphic to the free associative algebra $\mathbb{Z}\langle X\rangle$. We can write

$$
F=\bigoplus_{i \geqslant 1} \mathfrak{F}_{i}
$$

where $\mathfrak{F}_{i}$ is the $\mathbb{Z}$-module generated by Lie monomials of length $i$ in $X$. We then have $\left[\mathfrak{F}_{i}, \mathfrak{F}_{j}\right] \subseteq \mathfrak{F}_{i+j}$. Furthermore we can write

$$
\mathcal{U} F=\bigoplus_{i \geqslant 0} \mathfrak{A}_{i}
$$

where $\mathfrak{A}_{i}$ denotes the $\mathbb{Z}$-module generated by associative monomials of length $i$ in $X$ and $\mathfrak{A}_{0}=\mathbb{Z}$. Then we also have $\mathfrak{A}_{i} \mathfrak{A}_{j} \subseteq \mathfrak{A}_{i+j}$, and

$$
\mathfrak{F}_{1}=\mathfrak{A}_{1}=\bigoplus_{x \in X} \mathbb{Z} x .
$$

For $a, b \in F$ we can again write $[a, b]=a b-b a \in \mathcal{U} F$. This shows that the natural embedding $\iota: F \hookrightarrow \mathcal{U} F$ respects the grading, i.e. $\iota\left(\mathfrak{F}_{i}\right) \subseteq \mathfrak{A}_{i}$. 
Let now $\mathfrak{f}$ denote the augmentation ideal in $\mathcal{U} F$. Then $\mathfrak{f}=\bigoplus_{i \geqslant 1} \mathfrak{A}_{i}$, and more generally

$$
\mathfrak{f}^{n}=\bigoplus_{i \geqslant n} \mathfrak{A}_{i} .
$$

This means that $\mathfrak{f}^{n} \cap F$ consists of all polynomials whose homogeneous components are all of degree at least $n$, and which can be written as a Lie element. But then this Lie element must also be a sum of Lie monomials of degree at least $n$. By [37, p.12] these now can be written as a sum of left normed commutators of length at least $n$, which are precisely the generators of $\gamma_{n}(F)$.

Remark 3.4. If we want to study the dimension quotient $\delta_{n}(L) / \gamma_{n}(L)$ for a fixed $n$, we can always assume $L$ to be finitely generated, as in every element of $\delta_{n}(L) \backslash \gamma_{n}(L)$ only finitely many generators will appear. Also we can assume $L$ to be nilpotent of degree at most $n-1$, i.e. $\gamma_{n}(L)=0$. Now a finitely generated Lie $\operatorname{ring} L$ on $m$ generators with $\gamma_{n}(L)=0$ will be a quotient of $F / \gamma_{n}(F)$, where $F$ is the free Lie ring on the set of generators $\left\{X_{1}, \ldots, X_{m}\right\}$. But as an abelian group $F / \gamma_{n}(F)$ is finitely generated, so as abelian groups

$$
L \simeq \mathbb{Z}^{k} \oplus \bigoplus_{i \in I} \mathbb{Z} /\left(p_{i}^{k_{i}}\right)
$$

for some finite set $I$ and some (not necessarily distinct) primes $p_{i}$. Thus suppose $\delta_{n}(L) \neq 0$ and take $0 \neq x \in \delta_{n}(L)$. As $\delta_{n}(L)$ is a torsion module, there is an integer $c \in \mathbb{Z}$ such that $c x=0$, so $x$ has trivial torsion free part, and we can assume $k=0$, which means that in total we can assume the Lie ring $L$ to be finite. Furthermore suppose $x$ has non-zero components in $\mathbb{Z} /\left(p_{i}^{k_{i}}\right)$ and $\mathbb{Z} /\left(p_{j}^{k_{j}}\right)$ with $p_{i} \neq p_{j}$. Then $0 \neq p_{j}^{k_{j}} x$ still has non trivial component in $\mathbb{Z} /\left(p_{i}^{k_{i}}\right)$, but trivial component in $\mathbb{Z} /\left(p_{j}^{k_{j}}\right)$. Thus we can assume that all $p_{i}$ are equal, so that we can work in a Lie ring, whose underlying abelian group is a finite abelian $p$-group for some prime $p$.

For an associative ring $R$ (not necessarily with unit) we denote by $R^{n}$ the subring of $R$ generated by all products $x_{1} \cdots x_{n}$ with $x_{i} \in R$. We call $R$ nilpotent of degree $n$, if $R^{n}=0$.

Lemma 3.5. Let $L$ be a Lie ring. Then the following two statements are equivalent.

1. $\gamma_{n}(L)=L \cap \varpi^{n}(L)=0$,

2. L embeds into an associative ring, nilpotent of degree $n$, viewed as a Lie ring.

Proof. Suppose $L$ is a Lie ring with $\gamma_{n}(L)=0$. If $L \cap \varpi^{n}(L)=0$, then $L$ embeds into $\varpi(L) / \varpi^{n}(L)$, which is an associative ring, nilpotent of degree $n$.

On the other hand, suppose $L$ embeds into a ring $R$ with $R^{n}=0$. Then $\gamma_{n}(L)$ embeds into $R^{n}$, so $\gamma_{n}(L)=0$. Then this embedding extends to a map $\theta: \varpi(L) \rightarrow$ $R$, so we have the commutative diagram

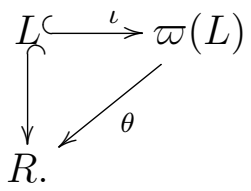


Thus $0=\operatorname{ker}(\theta \circ \iota)=L \cap \operatorname{ker}(\theta) \supseteq L \cap \varpi^{n}(L)$.

The following Theorem has been proven in [3]. We give an alternative proof here.

Theorem 3.6 (Bartholdi, Passi [3, Theorem 4.8]). For every Lie ring L, we have

$$
\delta_{2}(L)=\gamma_{2}(L)
$$

Proof. The statement is equivalent to proving that $\delta_{2}(L)=0$ for every abelian Lie ring.

So let $L$ be a finitely generated Lie ring, i.e. a finitely generated abelian group. Then we can write

$$
L \simeq \mathbb{Z} /\left(e_{1}\right) \oplus \cdots \oplus \mathbb{Z} /\left(e_{m}\right)
$$

with $e_{i} \mid e_{i+1}$ for every $i \in\{1, \ldots, m-1\}$. Denote the generator of the $i$-th summand by $X_{i}$. By Remark 2.26, we have

$$
\mathcal{U} L=\operatorname{Sym}(L) \simeq \mathbb{Z}\left[X_{1}, \ldots, X_{m}\right] /\left\langle e_{1} X_{1}, \ldots, e_{m} X_{m}\right\rangle .
$$

As $\varpi(L)=\left\langle X_{1}, \ldots, X_{m}\right\rangle$, we have $\varpi^{2}(L)=\left\langle X_{i} X_{j}\right\rangle$. Thus, we can uniquely write every $u \in L \cap \varpi^{2}(L)$ as

$$
u \equiv \sum_{i, j} a_{i j} X_{i} X_{j} \quad \bmod \varpi^{3}(L)
$$

with $0 \leqslant a_{i j}<\min \left\{e_{i}, e_{j}\right\}$. By Example 2.41 , as $u \in L$, we have $\Delta(u)=1 \otimes u+u \otimes 1$, and on the other hand $\Delta(u)=1 \otimes u+u \otimes 1+\sum_{i, j} a_{i j}\left(X_{i} \otimes X_{j}+X_{j} \otimes X_{i}\right)$. Therefore,

$$
\sum_{i, j} a_{i j}\left(X_{i} \otimes X_{j}+X_{j} \otimes X_{i}\right)=0 \in L \otimes L
$$

Now

$$
L \otimes L \simeq \bigoplus_{i, j}\left(\mathbb{Z} /\left(e_{i}\right) \otimes \mathbb{Z} /\left(e_{j}\right)\right)
$$

where the $(i, j)$-component is generated by $X_{i} \otimes X_{j}$. As $\mathbb{Z} /\left(e_{i}\right) \otimes \mathbb{Z} /\left(e_{j}\right) \simeq \mathbb{Z} /\left(\min \left\{e_{i}, e_{j}\right\}\right)$, the additive order of $X_{i} \otimes X_{j}$ is $\min \left\{e_{i}, e_{j}\right\}$, so every element of $L \otimes L$ can be uniquely expressed as $\sum_{i, j} c_{i j} X_{i} \otimes X_{j}$ with $0 \leqslant c_{i j}<\min \left\{e_{i}, e_{j}\right\}$. As the $a_{i j}$ satisfy this requirement, it follows that $a_{i j}=0$ for all $i, j$, and thus $u=0$.

Our strategy in the following will be to write a given Lie ring $L$ as quotient of a free Lie ring. As the Lie dimension subrings of a free Lie ring have been identified, this hopefully leads to results in general Lie rings.

Lemma 3.7. Let $L=F / R$ be a Lie ring, and let $n \geqslant 1$. Let $\mathfrak{r}$ and $\mathfrak{f}$ denote the ideals of $\mathcal{U} F$ generated by $R$ and $F$ respectively. Then

$$
\begin{aligned}
\gamma_{n}(L) & \simeq\left(\gamma_{n}(F)+R\right) / R, \\
\varpi^{n}(L) & \simeq\left(\mathfrak{f}^{n}+\mathfrak{r}\right) / \mathfrak{r}, \\
\delta_{n}(L) & \simeq\left(F \cap\left(\mathfrak{f}^{n}+\mathfrak{r}\right)\right) / R,
\end{aligned}
$$


and therefore

$$
\delta_{n}(L) / \gamma_{n}(L) \simeq\left(\left(\mathfrak{f}^{n}+\mathfrak{r}\right) \cap F\right) /\left(\gamma_{n}(F)+R\right) .
$$

Furthermore, there is an isomorphism

$$
\delta_{n}(L) / \gamma_{n}(L) \simeq \delta_{n}\left(F /\left(\gamma_{n}(F)+R\right)\right) .
$$

In particular, $\gamma_{n}(L)=\delta_{n}(L)$ if and only if $\gamma_{n}(F)+R=F \cap\left(\mathfrak{f}^{n}+\mathfrak{r}\right)$ for every presentation $L \simeq F / R$.

Proof. Because $[x+R, y+R]=[x, y]+R$ for $x, y \in F$ we see that $\gamma_{n}(L) \simeq$ $\gamma_{n}(F) /\left(R \cap \gamma_{n}(F)\right)$, which is isomorphic to $\left(R+\gamma_{n}(F)\right) / R$ by the isomorphism theorem.

For the second claim let $\pi: F \rightarrow F / R$ be the canonical projection. Extend $\pi$ to a map $\pi^{*}: \mathcal{U} F \rightarrow \mathcal{U}(F / R)$. Its kernel is $\mathfrak{r}$, so $\mathcal{U}(F / R) \simeq \mathcal{U} F / \mathfrak{r}$. Let now $\varepsilon: \mathcal{U} F \rightarrow \mathbb{Z}$ be the augmentation map, so that $\mathfrak{f}=$ ker $\varepsilon$. Let further $\varepsilon_{L}$ denote the augmentation map $\mathcal{U} F / \mathfrak{r} \rightarrow \mathbb{Z}$ with kernel $\varpi(F / R)$. Then $\varepsilon=\varepsilon_{L} \circ \pi^{*}$, so if $x+\mathfrak{r} \in \operatorname{ker}\left(\varepsilon_{L}\right)$, then $x \in \mathfrak{f}$, and therefore $\varpi(F / R) \simeq \mathfrak{f} / \mathfrak{r}$. Thus $\varpi^{n}(F / R) \simeq$ $\mathfrak{f}^{n} /\left(\mathfrak{r} \cap \mathfrak{f}^{n}\right) \simeq\left(\mathfrak{f}^{n}+\mathfrak{r}\right) / \mathfrak{r}$ using again the isomorphism theorem.

Thus, if for $f \in F$ we have $f+\mathfrak{r} \in \varpi^{n}(F / R)$, then $F \cap\left(\pi^{*}\right)^{-1}(f+\mathfrak{r}) \subseteq\left(\mathfrak{f}^{n}+\mathfrak{r}\right) \cap F$, and the third assertion follows.

For the proof of the last isomorphism, note that by the above we have $\delta_{n}(F / R) \simeq$ $\left(\left(\mathfrak{f}^{n}+\mathfrak{r}\right) \cap F\right) / R$ and $\gamma_{n}(F / R) \simeq\left(\gamma_{n}(F)+R\right) / R$. Thus by the second isomorphism theorem

$\delta_{n}(F / R) / \gamma_{n}(F / R) \simeq\left(\left(\left(\mathfrak{f}^{n}+\mathfrak{r}\right) \cap F\right) / R\right) /\left(\left(\gamma_{n}(F)+R\right) / R\right) \simeq\left(F \cap\left(\mathfrak{f}^{n}+\mathfrak{r}\right)\right) /\left(\gamma_{n}(F)+R\right)$,

and on the other hand

$$
\delta_{n}\left(F /\left(\gamma_{n}(F)+R\right)\right) \simeq\left(F \cap\left(\mathfrak{r}+\mathfrak{f}^{n}+\mathfrak{f}^{n}\right)\right) /\left(\gamma_{n}(F)+R\right),
$$

completing the proof.

Proposition 3.8. Let $L \simeq F / R$ be a Lie ring. Then $\delta_{n}(L)=\gamma_{n}(L)$ for a given $n \geqslant 1$, if and only if $F \cap\left(\mathfrak{f}^{n}+\mathfrak{f r}\right) \subseteq \gamma_{n}(F)+R$.

Proof. Clearly, if $\delta_{n}(L)=\gamma_{n}(L)$, then $F \cap\left(\mathfrak{f}^{n}+\mathfrak{f} \mathfrak{r}\right) \subseteq F \cap\left(\mathfrak{f}^{n}+\mathfrak{r}\right)=\gamma_{n}(F)+R$.

Now suppose $F \cap\left(\mathfrak{f}^{n}+\mathfrak{f r}\right) \subseteq \gamma_{n}(F)+R$, and let $u=u_{1}+u_{2} \in F \cap\left(\mathfrak{f}^{n}+\mathfrak{r}\right)$ with $u_{1} \in \mathfrak{f}^{n}$ and $u_{2} \in \mathfrak{r}$. Then $u_{2}=\sum_{j} v_{j} r_{j}$ with $v_{j} \in \mathcal{U} F$ and $r_{j} \in R$. Now write $v_{j}=\varepsilon\left(v_{j}\right)+v_{j}^{\prime}$ with $v_{j}^{\prime} \in \mathfrak{f}$ and $\varepsilon\left(v_{j}\right)=: n_{j} \in \mathbb{Z}$. Then $u_{2} \equiv \sum_{j} n_{j} r_{j} \bmod \mathfrak{f r}$, and thus $u \equiv \sum_{j} n_{j} r_{j} \bmod \mathfrak{f}^{n}+\mathfrak{f r}$, so $u-\sum_{j} n_{j} r_{j} \in F \cap\left(\mathfrak{f}^{n}+\mathfrak{f r}\right) \subseteq \gamma_{n}(F)+R$ by assumption. As $\sum_{j} n_{j} r_{j} \in R$, we conclude that $u \in \gamma_{n}(F)+R$, as required.

As for groups, it will be helpful to have some control of the presentation of a Lie ring.

Definition 3.9. Let $L=F / R$ be a presentation of a finitely generated Lie ring, where $F$ is generated by $X=\left\{X_{1}, \ldots, X_{m}\right\}$. We call the presentation pre-abelian, if $R$ is of the form $R=\left\langle e_{1} X_{1}+\xi_{1}, \ldots, e_{m} X_{m}+\xi_{m}, \xi_{m+1}, \xi_{m+2}, \ldots\right\rangle$, where $e_{i}$ are integers with $e_{i} \mid e_{i+1}$ for every $i<m$, and $\xi_{i} \in \gamma_{2}(F)$ for every $i$. 
Lemma 3.10. Let $L=F / R$ be a finitely generated Lie ring. Then there exists a set of free generators $X=\left\{X_{1}, \ldots, X_{m}\right\}$ of $F$, such that $L$ is given by a pre-abelian presentation.

Proof. Let $L$ be a Lie ring generated by $m$ elements as a Lie ring. Then $L / \gamma_{2}(L) \simeq$ $F /\left(\gamma_{2}(F)+R\right)$ is a finitely generated abelian group on the generators of $L$. By the elementary divisor theorem there is a generating set $X=\left\{X_{1}, \ldots, X_{m}\right\}$ of $L / \gamma_{2}(L)$ and elementary divisors $e_{1}, \ldots, e_{m}$ with $e_{i} \mid e_{i+1}$ for every $i<m$, such that

$$
L / \gamma_{2}(L) \simeq \mathbb{Z} /\left(e_{1}\right) \oplus \cdots \oplus \mathbb{Z} /\left(e_{m}\right),
$$

where $X_{i}$ generates the $i$-th direct summand. This means that $\gamma_{2}(F)+R=$ $\left\langle e_{1} X_{1}, \ldots, e_{m} X_{m}, \gamma_{2}(F)\right\rangle$, so $R$ can be given in the desired way.

As in groups, also the third terms of the two series coincide, so in all we have:

Theorem 3.11 (Bartholdi, Passi [3, Theorem 4.3]). For any Lie ring L and $n \leqslant 3$ we have

$$
\delta_{n}(L)=\gamma_{n}(L)
$$

However, for $n \geqslant 4$ equality does not always hold, as the following examples show (for $n=4$, this is exactly the example given in [3]).

Example 3.12. Let $L$ be a Lie ring generated by the set $\{r, a, b, c\}$ and fix $n \geqslant 4$. Define $x_{0}=y_{0}=z_{0}=r$ and $x_{i}=\left[x_{i-1}, a\right], y_{i}=\left[y_{i-1}, b\right], z_{i}=\left[z_{i-1}, c\right]$. Suppose $L$ is given by the following set of relations.

$$
\begin{aligned}
& 2^{2 n-1} r=0,2^{n+2} a=4 y_{n-3}+2 z_{n-3}, 2^{n} b=-4 x_{n-3}+z_{n-3}, 2^{n-2} c=-2 x_{n-3}-y_{n-3}, \\
& z_{n-2}=4 y_{n-2}, y_{n-2}=4 x_{n-2} \text {, } \\
& x_{n-1}=y_{n-1}=z_{n-1}=0 \text {, } \\
& {[a, b, u]=[b, c, u]=[a, c, u]=0 \text { for all } u \in L,} \\
& {\left[x_{i}, b\right]=\left[x_{i}, c\right]=\left[y_{i}, a\right]=\left[y_{i}, c\right]=\left[z_{i}, a\right]=\left[z_{i}, b\right]=0 \text { for all } i \geqslant 1,} \\
& {\left[x_{i}, x_{j}\right]=\left[x_{i}, y_{j}\right]=\left[x_{i}, z_{j}\right]=\left[y_{i}, y_{j}\right]=\left[y_{i}, z_{j}\right]=\left[z_{i}, z_{j}\right]=0 \text { for all } i, j \geqslant 0 \text {. }}
\end{aligned}
$$

Then $L$ is a metabelian Lie ring with $\gamma_{n}(L)=0$, and

$$
0 \neq g=2^{2 n-1}[a, b]+2^{2 n-2}[a, c]+2^{2 n-3}[b, c] \in \delta_{2 n-4}(L) \subseteq \delta_{n}(L) .
$$

Proof. Observe first that in $L$ all non-zero commutators in $r, a, b, c$ of length $i \geqslant 3$ are $x_{i-1}, y_{i-1}, z_{i-1}$ by relations (3.5), (3.6) and (3.7). As $x_{n-1}=y_{n-1}=z_{n-1}=$ 
0 , we observe that $\gamma_{n}(L)=0$. Also, as an abelian group $L^{\prime}$ is generated by $[a, b],[a, c],[b, c], x_{i}, y_{i}, z_{i}$ for $1 \leqslant i \leqslant n-2$, which commute pairwise by (3.5) and (3.7), so $L$ is metabelian.

Now write $g$ as an element of $\mathcal{U} L$ as

$$
g=a\left(2^{2 n-1} b+2^{2 n-2} c\right)+b\left(2^{2 n-3} c-2^{2 n-1} a\right)-c\left(2^{2 n-1} a+2^{2 n-3} b\right) .
$$

Applying the relations 3.2 yields

$$
a\left(2^{2 n-1} b+2^{2 n-2} c\right)=a\left(-2^{n+2} x_{n-3}-2^{n} y_{n-3}+2^{n} z_{n-3}\right),
$$

and as e.g. $2^{n+2} x_{n-3}=4\left[x_{n-4}, y_{n-3}\right]+2\left[x_{n-4}, z_{n-3}\right]=0$ we obtain $a\left(2^{2 n-1} b+\right.$ $\left.2^{2 n-2} c\right) \in \varpi^{2 n-4}$. Proceeding similarly we obtain

$$
b\left(2^{2 n-3} c-2^{2 n-1} a\right)=b\left(-2^{n} x_{n-3}+2^{n-2} z_{n-3}\right) .
$$

Now $2^{n-2} z_{n-3}=0$, and also $2^{n} b x_{n-3} \in \varpi^{2 n-4}(L)$. Finally

$$
c\left(2^{2 n-1} a+2^{2 n-3} b\right)=2^{n-2} c\left(-2 x_{n-3}+y_{n-3}+z_{n-3}\right) \in \varpi^{2 n-4}(L),
$$

and therefore $g \in \delta_{2 n-4}(L)$.

It remains to prove $g \neq 0$. Note that we have

$g=2^{2 n-1}[a, b]+2^{2 n-2}[a, c]+2^{2 n-3}[b, c]=2^{n-1} y_{n-2}+2^{n-3} z_{n-2}+2^{n-1} y_{n-2}=2^{n-3} z_{n-2}$.

Note also that the relation $z_{n-2}=4 y_{n-2}$ follows from (3.2) and (3.6) by applying the Lie bracket with $c$ to $2^{n} b=-4 x_{n-3}+z_{n-3}$. Therefore we can omit that relation.

Now consider the free Lie ring $F$ generated by $r, a, b, c$. Denote by $R$ the ideal generated by the relators corresponding to the relations above. To prove that $g \neq 0$ in $L$, it suffices to prove that $2^{n-3} z_{n-2} \notin R$. So suppose that $2^{n-3} z_{n-2} \in R$. Then there exist integers $a_{0}, \ldots, a_{7}$ such that modulo $\gamma_{n}(F)$

$$
\begin{aligned}
2^{n-3} z_{n-2} & =2^{n-2} a_{0} z_{n-2}+a_{1}\left(2^{n+2}[a, c]-4\left[y_{n-3}, c\right]-2 z_{n-2}\right) \\
& +a_{2}\left(2^{n}[b, c]+4\left[x_{n-3}, c\right]-z_{n-2}\right)+a_{3}\left(2^{n-2}[a, c]-2 x_{n-2}-\left[y_{n-3}, a\right]\right) \\
& +a_{4}\left(2 n-2[b, c]-2\left[x_{n-3}, b\right]-y_{n-2}\right)+a_{5}\left(y_{n-2}-4 x_{n-2}\right) \\
& +a_{6}\left(2^{n+2}[a, b]-4 y_{n-2}-2\left[z_{n-3}, b\right]\right) \\
& +a_{7}\left(2^{n}[a, b]-4 x_{n-2}+\left[z_{n-3}, a\right]\right)+q,
\end{aligned}
$$

where $q \in R$ does not involve $[a, b],[b, c],[a, c], x_{n-2}, y_{n-2}, z_{n-2}$. Comparing coefficients of basic commutators leads to the following set of linear equations.

$$
\left\{\begin{array}{rlrl}
-2 a_{1}-a_{2} & & +2^{n-2} a_{0} & =2^{n-3} \\
16 a_{1}+a_{3} & & =0 \\
4 a_{2}+a_{4} & & =0 \\
& -a_{4}+a_{5}-4 a_{6} & \\
-a_{3}-2 a_{5}-2 a_{7} & =0 \\
& & 4 a_{6}+a_{7} & =0 .
\end{array}\right.
$$


From the last three equations we obtain $a_{3}=-2 a_{4}$. As $a_{4}=-4 a_{2}$, we obtain $a_{3}=8 a_{2}$, and therefore, because of the second equation, $2 a_{1}+a_{2}=0$. But now, the first equation becomes $2^{n-2} a_{0}=2^{n-3}$, which does not have an integer solution.

If $L$ is a graded Lie ring generated in degree 1, the dimension series and lower central series will coincide, as the following theorem shows. Therefore, taking Gupta's examples 2.77 of groups without the dimension property, and defining the Lie ring by 2.16 from them, will not lead to Lie rings without dimension property.

Theorem 3.13. Let $\mathfrak{a} \subseteq \mathcal{U} F$ be a homogeneous ideal of a free associative algebra. Define $A:=F \cap \mathfrak{a}$. Then

$$
F \cap\left(\mathfrak{f}^{n}+\mathfrak{a}\right)=\gamma_{n}(F)+A
$$

for every $n \in \mathbb{N}$.

Proof. It is clear that $\gamma_{n}(F)+A \subseteq F \cap\left(\mathfrak{f}^{n}+\mathfrak{a}\right)$. For the reverse inclusion, assume that $a \in F \cap\left(\mathfrak{f}^{n}+\mathfrak{a}\right)$. Then modulo $\mathfrak{f}^{n}$ we can uniquely write $a=a_{0}+\cdots+a_{n-1}$, where $a_{i}$ is the homogeneous component of degree $i$ of $a$. As $\mathfrak{a}$ is homogeneous, it follows that $a_{i} \in \mathfrak{a}$ for all $i \leqslant n-1$. On the other hand, as $a \in F$, we may uniquely write $a \equiv f_{1}+\cdots+f_{n-1} \bmod \gamma_{n}(F)$ with $f_{i}$ homogeneous of degree $i$. Thus $a_{0}=0$ and $a_{i}=f_{i}$ for $i \geqslant 1$, and therefore $a_{i} \in F \cap \mathfrak{a}=A$. Thus $a \in A+\gamma_{n}(F)$, as desired.

Corollary 3.14. Let $L$ be a graded Lie ring generated in degree 1 . Then $\delta_{n}(L)=$ $\gamma_{n}(L)$ for all $n \in \mathbb{N}$.

Proof. If $L$ is graded and generated in degree 1 , we can write $L=F / R$, where $R$ is a homogeneous ideal of $F$. It follows that $\mathfrak{r}$ is homogeneous, and by Theorem 3.13 we have $F \cap\left(\mathfrak{f}^{n}+\mathfrak{r}\right)=\gamma_{n}(F)+R$.

\subsection{Sjogren's Theorem for the Lie Dimension Problem}

We now analyze $n$-th dimension quotient $\delta_{n}(L) / \gamma_{n}(L)$. It turns out that the proof by Cliff and Hartley [8] for Sjogren's Theorem 2.78 can be translated to Lie rings with the same constants as in Sjogren's Theorem for groups.

Theorem 3.15. Define $b_{m}=\operatorname{lcm}(1, \ldots, m)$, and set

$$
c_{n}=\prod_{i=1}^{n-2} b_{i}^{\left(\begin{array}{c}
n-2 \\
i
\end{array}\right)} .
$$

Then $c_{n} \delta_{n}(L) \subseteq \gamma_{n}(L)$ for every Lie ring $L$. 
Write again $L=F / R$ with a free Lie ring $F$ and an ideal $R$ of $F$. Our strategy is now to define a descending series of ideals of $F$ such that we can bound the exponents of the quotients to obtain a bound for the exponent of $\delta_{n}\left(\gamma_{n}(F) /\left(\gamma_{n}(F)+\right.\right.$ $R)$ ). For this purpose define for $k \in \mathbb{N}$ the subalgebra

$$
R(k)=[R, F, \ldots, F]
$$

with $k-1$ appearances of $F$, where the commutator is left-normed, and

$$
\mathfrak{r}(k)=\sum_{i=0}^{k-1} \mathfrak{f}^{i} \mathfrak{r} \mathfrak{f}^{k-1-i} \subseteq \mathcal{U} F .
$$

Lemma 3.16. We have

$$
R(k) \subseteq \mathfrak{r}(k) \cap \gamma_{k}(F),
$$

so in particular

$$
\mathcal{U} F \cdot R(k) \subseteq \mathfrak{r}(k)
$$

Proof. For $k=1$ the statement is obvious and $R(k+1)=[R(k), F]$ is generated by all commutators of the form $[x, y]$ with $x \in R(k)$ and $y \in F$. In $\mathcal{U} F$ we can again write $[x, y]=x y-y x$, and by the induction hypothesis we have $x \in$ $\mathfrak{r} \mathfrak{f}^{k-1}+\mathfrak{f} \mathfrak{r} \mathfrak{f}^{k-2}+\ldots+\mathfrak{f}^{k-1} \mathfrak{r}$ and $y \in \mathfrak{f}$ giving the first claim.

By taking ideals on both sides we get

$$
\mathcal{U} F \cdot R(k) \subseteq \mathcal{U} F\left(\mathfrak{r}(k) \cap \gamma_{k}(F)\right)=\mathfrak{r}(k) \cap \mathfrak{f}^{k}=\mathfrak{r}(k) .
$$

Now for $k \leqslant n$ define

$$
E_{n, k}=F \cap\left(\mathfrak{f}^{n}+\mathfrak{r}(k)\right)=\gamma_{k}(F) \cap\left(\mathfrak{f}^{n}+\mathfrak{r}(k)\right),
$$

where the last equality holds because $k \leqslant n$ and thus $\mathfrak{f}^{n} \subseteq \mathfrak{f}^{k}$ and further $\mathfrak{r}(k) \subseteq \mathfrak{f}^{k}$ and $\mathfrak{f}^{k} \cap F=\delta_{k}(F)=\gamma_{k}(F)$. Then $E_{n, k} \subseteq E_{m, k}$ for $m \leqslant n$ and $E_{n, k} \subseteq E_{n, \ell}$ for $\ell \leqslant n$, and also $E_{n, n}=\gamma_{n}(F)$.

We have $R(k) \subseteq E_{n, k}$, so $E_{n, k}=E_{n, k} \cap \gamma_{k}(F)$ and $E_{n, k} \cap \gamma_{n}(F)=\gamma_{n}(F)$. For $k \leqslant j \leqslant n$ set

$$
E_{n, j, k}=\left(E_{n, k} \cap \gamma_{j}(F)\right)+R(k),
$$

Then $E_{n, k, k}=E_{n, k}$ and $E_{n, n, k}=\gamma_{n}(F)+R(k)$, and we get the following descending series of ideals of $F$ :

$$
E_{n, k}=E_{n, k, k} \supseteq E_{n, k+1, k} \supseteq \ldots \supseteq E_{n, n-1, k} \supseteq E_{n, n, k}=\gamma_{n}(F)+R(k) .
$$

By Lemma 3.7 we see that $E_{n, 1} /\left(\gamma_{n}(F)+R\right)=\delta_{n}\left(F /\left(\gamma_{n}(F)+R\right)\right)=\delta_{n}(L) / \gamma_{n}(L)$ with $L=F / R$, so it suffices to bound the exponents of the quotients $E_{n, j, k} / E_{n, j+1, k}$ for $k \leqslant j<n$ to obtain a bound for the exponent of $\delta_{n}(L) / \gamma_{n}(L)$. 
Lemma 3.17. Let $k, j, n$ be integers with $k \leqslant j<n$. Then the inclusion of $E_{n, j, k}$ into $E_{j+1, j, k}$ induces an embedding

$$
E_{n, j, k} / E_{n, j+1, k} \hookrightarrow E_{j+1, j, k} /\left(\gamma_{j+1}(F)+R(k)\right) .
$$

Proof. We have to show that $E_{n, j, k} \cap\left(\gamma_{j+1}(F)+R(k)\right)=E_{n, j+1, k}$. As $E_{n, j+1, k}=$ $\left(E_{n, k} \cap \gamma_{j+1}(F)\right)+R(k) \subseteq\left(E_{n, k} \cap \gamma_{j}(F)\right)+R(k)$ and also $\left(E_{n, k} \cap \gamma_{j+1}(F)\right)+R(k) \subseteq$ $\gamma_{j+1}(F)+R(k)$, it is clear that $E_{n, j+1, k} \subseteq\left(\left(E_{n, k} \cap \gamma_{j}(F)\right)+R(k)\right) \cap\left(\gamma_{j+1}(F)+\right.$ $R(k))=E_{n, j, k} \cap\left(\gamma_{j+1}(F)+R(k)\right)$.

For the reverse inclusion note that by Lemma 3.16 we have $R(k) \subseteq \mathfrak{r}(k) \cap \gamma_{k}(F) \subseteq$ $\left(\mathfrak{f}^{n}+\mathfrak{r}(k)\right) \cap \gamma_{k}(F)=E_{n, k}$, so by the modular law

$$
E_{n, j+1, k}=\left(E_{n, k} \cap \gamma_{j+1}(F)\right)+R(k)=E_{n, k} \cap\left(\gamma_{j+1}(F)+R(k)\right) .
$$

Now we get $E_{n, k} \cap\left(\gamma_{j+1}(F)+R(k)=\left(E_{n, k}+R(k)\right)\right) \cap\left(\gamma_{j+1}(F)+R(k)\right) \geqslant\left(\left(E_{n, k} \cap\right.\right.$ $\left.\left.\gamma_{j}(F)\right)+R(k)\right) \cap\left(\gamma_{j+1}(F)+R(k)\right)=E_{n, j, k} \cap\left(\gamma_{j+1}(F)+R(k)\right)$, completing the proof.

Now for $k \leqslant j$, let $a_{j, k}$ denote the exponent of $\left(\left(E_{j, k} \cap \gamma_{j-1}(F)\right)+R(k)\right) /\left(\gamma_{j}(F)+\right.$ $R(k))$. Then because of the above inclusion and the characterization of $\delta_{n}(L) / \gamma_{n}(L)$ we get

Lemma 3.18. The exponent of $E_{n, k} /\left(\gamma_{n}(F)+R(k)\right)$ divides $\prod_{j=k+1}^{n} a_{j, k}$. Thus the exponent of $\delta_{n}(L) / \gamma_{n}(L)=E_{n, 1} /\left(\gamma_{n}(F)+R\right)$ divides $\prod_{j=2}^{n} a_{j, 1}$.

As $E_{n, n}=\gamma_{n}(F)=\gamma_{n}(F) \cap \gamma_{n-1}(F)$ and $R(n) \subseteq \gamma_{n}(F)$ we obtain $a_{n, n}=1$. The next exponent $a_{n, n-1}$ can also be computed.

Lemma 3.19. We have

$$
a_{n, n-1}=1 .
$$

Proof. Let $\left\{X_{i}: i \in I\right\}$ be a set of free generators of $F$. Then $\mathcal{U} F$ is isomorphic to $\mathbb{Z}\left\langle X_{i}\right\rangle$, i.e. the ring of non-commuting polynomials in the $X_{i}$ with integer coefficients. Then $\mathfrak{f}$ is the ideal generated by the $X_{i}$, and more generally $\mathfrak{f}^{n}$ is the ideal generated by all monomials in the $X_{i}$ of degree $n$. Thus modulo $\mathfrak{f}^{n}$ every element of $\mathcal{U F}$ can be uniquely written as a linear combination of monomials of degree at most $n-1$.

Choose now $x \in\left(E_{n, n-1} \cap \gamma_{n-1}(F)\right)+R(n-1)=E_{n, n-1}$. We show $x \in \gamma_{n}(F)+$ $R(n-1)$. The natural embedding $F \hookrightarrow \mathcal{U} F$ embeds $\gamma_{k}(F)$ into $\mathfrak{f}^{k}$ for every $k$.

As only finitely many $X_{i}$ appear in the expression of $x$ we may assume that $I$ is finite. Set $V=\bigoplus_{i \in I} \mathbb{Z} X_{i}$ and $W=\left(\mathfrak{r}+\mathfrak{f}^{2}\right) \cap V$. The ideal $\mathfrak{r}(n-1)$ is generated by elements of the form $X_{1} \cdots X_{i-1} r X_{i+1} \cdots X_{n-1}$, where $r \in \mathfrak{r}$. Let $V_{j}$ be the $\mathbb{Z}$-module consisting of the homogeneous polynomials of degree $j$ and write

$$
\mathcal{U} F=\bigoplus_{j=0}^{\infty} V_{j} .
$$


Then $V_{1}=V$ and $V_{0} \cap \mathfrak{r} \subseteq V_{0} \cap \mathfrak{f}=\{0\}$, so the homogeneous part of $r$ of degree zero in the above expression is zero, and we can write

$$
r=\sum_{j>0} r_{j}
$$

with $r_{j} \in V_{j}$. As $V_{j} \subseteq \mathfrak{f}^{j}$, we get

$$
\begin{aligned}
X_{1} \cdots X_{i-1} r X_{i+1} \cdots X_{n-1} & =\sum_{j>0} X_{1} \cdots X_{i-1} r_{j} X_{i+1} \cdots X_{n-1} \\
& \equiv X_{1} \cdots X_{i-1} r_{1} X_{i+1} \cdots X_{n-1} \quad \bmod \mathfrak{f}^{n},
\end{aligned}
$$

but $r_{1}=r-\sum_{j \geqslant 2} r_{j} \in \mathfrak{r}+\mathfrak{f}^{2}$. Thus modulo $\mathfrak{f}^{n}$ an element of $\mathfrak{r}(n-1)$ is congruent to a linear combination of monomials of degree $n-1$ in which $n-2$ factors are of the form $X_{i}$ and the other is in $W$.

The elementary divisor theorem gives us a basis $y_{1}, \ldots, y_{t}$ of $V$, such that for an $s \leqslant t$ and integers $e_{1}, \ldots, e_{s}$ with $e_{i} \mid e_{i+1}$ for $1 \leqslant i<s, z_{i}=e_{i} y_{i},(1 \leqslant i \leqslant s)$, form a basis of $W$.

Now the $y_{i}$ are in $V$ and thus they are Lie elements of $\mathcal{U} F$, i.e. elements of $F$. Thus $x \in \gamma_{n-1}(F)$ can be uniquely written as

$$
x \equiv \xi_{1}+\cdots+\xi_{t-1} \bmod \mathfrak{f}^{n},
$$

where each $\xi_{j}$ is a linear combination of basic commutators of weight $n-1$ in the $y_{i}$ involving $y_{j}$ and only those $y_{i}$ with $i \geqslant j$.

On the other hand we have $x \in \mathfrak{r}(n-1)+\mathfrak{f}^{n}$, so modulo $\mathfrak{f}^{n}$ we can (again uniquely) write $x$ as a linear combination of associative monomials

$$
x \equiv \eta_{1}+\cdots+\eta_{s} \bmod \mathfrak{f}^{n},
$$

where each $\eta_{j}$ is a linear combination of monomials in $y_{i}$ and $z_{i}$ of degree $n-1$ involving exactly one $z_{i}$, involving $y_{j}$ or $z_{j}$ and only those $y_{i}$ and $z_{i}$ with $i \geqslant j$. As $e_{j}$ divides all the $e_{i}$ with $i \geqslant j$, we can write $\eta_{j}=e_{j} \eta_{j}^{\prime}$. Because basic commutators of weight $n-1$ lead to monomials of degree $n-1$ we obtain

$$
\xi_{j}= \begin{cases}e_{j} \eta_{j}^{\prime} & \text { if } j \leqslant s \\ 0 & \text { if } j>s .\end{cases}
$$

Thus we can write $\xi_{j}=e_{j} \xi_{j}^{\prime}$ for $j \leqslant s$, where $\xi_{j}^{\prime}$ is again a basic commutator involving $y_{j}$ and only those $y_{i}$ with $i \geqslant j$. By bilinearity of the Lie bracket we can write $\xi_{j}$ as a linear combination of commutators of weight $n-1$ in $z_{j}$ and $y_{j}, \ldots, y_{t}$ involving exactly one $z_{j}$.

Now $z_{i} \in \mathfrak{r}+\mathfrak{f}^{2} \cap V \subseteq \mathfrak{r}+\mathfrak{f}^{2} \cap F$, and by Lemma 3.7 and Theorem 3.11 we have $\left(\mathfrak{r}+\mathfrak{f}^{2}\right) \cap F=R+\gamma_{2}(F)$, so we can write $z_{i}=v_{i}+f_{i}$ with $v_{i} \in R$ and $f_{i} \in \gamma_{2}(F) \subseteq \mathfrak{f}^{2}$, so we find $v_{i} \in R$ with $v_{i} \equiv z_{i} \bmod \mathfrak{f}^{2}$. So a Lie product of length $n-1$ in the $y_{j}$ and $z_{j}$ involving one $z_{j}$ is unchanged modulo $f^{n}$ if we replace $z_{j}$ by $v_{j}$. So $x$ is congruent to a linear combination of Lie products of weight $n-1$ in $y_{j}$ and $v_{j}$ involving exactly one $v_{j}$. As $y_{j} \in F$ and $v_{j} \in R$, there is a $w \in R(n-1)$ with $x \equiv w$ $\bmod \mathfrak{f}^{n}$. Altogether we have $x-w \in F \cap \mathfrak{f}^{n}=\gamma_{n}(F)$, so $x \in \gamma_{n}(F)+R(n-1)$, as desired. 
It remains to prove the following crucial Lemma, which allows us to bound the exponents of the given quotients.

Lemma 3.20. Let $F$ be freely generated by $\left\{X_{i}: i \in I\right\}$. If $k<n$ and $x \in$ $E_{n, k} \cap \gamma_{n-1}(F)$, then there exists $r \in R(k)$ such that $b_{k} x+r \in E_{n, k+1}$, where $b_{k}=\operatorname{lcm}(1, \ldots, k)$.

We will now set up the machinery to prove the above Lemma. Let $B$ be the free associative algebra on a countably infinite alphabet $Y=\left\{y_{1}, y_{2}, \ldots\right\}$ over $\mathbb{Q}$. For $\nu \geqslant 1$ set

$$
\theta_{\nu}\left(y_{1}, \ldots, y_{t}\right)=\frac{(-1)^{\nu+1}}{\nu}\left(\left(y_{1}+1\right) \cdots\left(y_{t}+1\right)-1\right)^{\nu},
$$

and let $\sigma_{\nu}\left(y_{1}, \ldots, y_{t}\right)$ be the sum of those monomials of $\theta_{\nu}\left(y_{1}, \ldots, y_{t}\right)$ that involve all the generators $y_{1}, \ldots, y_{t}$, and let further $\hat{\sigma}_{\nu}\left(y_{1}, \ldots, y_{t}\right)$ be the sum of those monomials in $\sigma_{\nu}\left(y_{1}, \ldots, y_{t}\right)$ that involve every generator exactly once, i.e. the homogeneous component of degree $t$ of $\sigma_{\nu}$. Set now $A:=\mathcal{U} F \otimes \mathbb{Q}$, i.e. the free associative algebra over $\mathbb{Q}$ on $X=\left\{X_{i}: i \in I\right\}$, and define the following maps on the monomials of $A$ and extend them by linearity:

$$
\begin{aligned}
\psi_{\nu}\left(X_{i_{1}} \cdots X_{i_{t}}\right) & =\left.\sigma_{\nu}\left(y_{1}, \ldots, y_{t}\right)\right|_{y_{j}=X_{i_{j}}}, \\
\hat{\psi}_{\nu}\left(X_{i_{1}} \cdots X_{i_{t}}\right) & =\left.\hat{\sigma}_{\nu}\left(y_{1}, \ldots, y_{t}\right)\right|_{y_{j}=X_{i_{j}}}, \\
\psi & =\sum_{\nu=1}^{\ell} \psi_{\nu}, \\
\hat{\psi} & =\sum_{\nu=1}^{\ell} \hat{\psi}_{\nu},
\end{aligned}
$$

where $\ell$ is a fixed integer. Then $b_{\ell} \psi$ and $b_{\ell} \hat{\psi}$ are module endomorphisms of $\mathcal{U} F$, and obviously

$$
\psi\left(X_{i_{1}} \cdots X_{i_{t}}\right)=\hat{\psi}\left(X_{i_{1}} \cdots X_{i_{t}}\right)+u
$$

where $u$ consists only of terms of degree at least $t+1$. We also see easily that

$$
b_{k} \psi(a) \in \mathcal{U} F
$$

The maps $\psi$ and $\hat{\psi}$ have the following important properties.

Lemma 3.21 (Cliff, Hartley [8, Lemma 3.1]). Let $A$ be as above. Fix $j \in I$ and a sequence $\left(j_{1}, \ldots, j_{r}\right)$ in $I$ and let $\delta: A \rightarrow A$ be the algebra endomorphism that fixes all the $X_{i}$ for $i \neq j$ and maps $X_{j}$ to $\left(X_{j_{1}}+1\right) \cdots\left(X_{j_{r}}+1\right)-1$. Then for $a$ monomial $\xi \in A$, which involves $X_{j}$ exactly once, we have

$$
\delta \psi_{\nu}(\xi)=\psi_{\nu} \delta(\xi)
$$

so in particular

$$
\delta \psi(\xi)=\psi \delta(\xi)
$$


Lemma 3.22 (Cliff, Hartley [8, Lemma 3.2]). The map $\hat{\psi}$ has the following properties.

1. $\hat{\psi}(a)$ is a Lie element of A for every a of degree at most $\ell$,

2. $\hat{\psi}(a)=$ a for every Lie element a of $A$.

Let $S$ denote the semigroup generated by the $X_{i}$ with the circle operation

$$
a \circ b=a b+a+b=(a+1)(b+1)-1 .
$$

We want to show that every Lie element can be written as sum of elements of $S$, so that an algebra endomorphism of $F$ sending each $X_{i}$ for $i \neq j$ to itself and $X_{j}$ to a Lie element will also commute with $\psi_{\nu}$ and $\psi$ by Lemma 3.21. We first of all recall that according to [37, p. 12] every Lie monomial can be written as a sum of left normed commutators.

Now define the Pólya action of the symmetric group $S_{n}$ on monomials of degree $n$ by

$$
X_{i_{1}} \cdots X_{i_{n}} \sigma=X_{i_{\sigma(1)}} \cdots X_{i_{\sigma(n)}}
$$

for $X_{i_{j}} \in X$ and $\sigma \in S_{n}$ and extend this action to the group algebra $\mathbb{Q} S_{n}$ by linearity. Then by [12, p. 314] we get:

Lemma 3.23. For $X_{i_{1}}, \ldots X_{i_{n}} \in X$ we have

$$
\left[X_{i_{1}}, \ldots, X_{i_{n}}\right]=X_{i_{1}} \cdots X_{i_{n}}\left(1-\tau_{2}\right) \cdots\left(1-\tau_{n}\right),
$$

where $\tau_{i}$ is the backwards $i$-cycle, i.e. $\tau_{i}=(i, i-1, \ldots, 1)$.

Now for a (possibly empty) subsequence $K$ of $(2, \ldots, n)$ let

$$
\tau_{K}:=\prod_{i \in K} \tau_{i}
$$

Note that we then have

$$
\left(1-\tau_{2}\right) \cdots\left(1-\tau_{n}\right)=\sum_{K}(-1)^{|K|} \tau_{K}
$$

where the sum is taken over all subsequences $K$ of $(2, \ldots, n)$ and $|K|$ denotes the length of $K$. Furthermore, for $X_{i} \in X$, set $\tilde{X}_{i}=X_{i}+1$, and for $\sigma \in S_{n}$ set

$$
\tilde{X}_{\sigma}=\tilde{X}_{\sigma(1)} \cdots \tilde{X}_{\sigma(n)} \text {. }
$$

Now we are in a position to give an explicit formula how to write any left normed commutator as sum of elements of $S$.

Lemma 3.24. For $n \geqslant 2$ we have

$$
\left[X_{1}, \ldots, X_{n}\right]=\sum_{K}(-1)^{|K|} \tilde{X}_{\tau_{K}}
$$

where the sum is taken over all (possibly empty) subsequences $K$ of $(2, \ldots, n)$. 
Proof. We prove by induction on $n$. For $n=2$ we see that $\left[X_{1}, X_{2}\right]=X_{1} X_{2}-X_{2} X_{1}=X_{1} X_{2}+X_{1}+X_{2}+1-\left(X_{2} X_{1}+X_{2}+X_{1}+1\right)=\tilde{X}_{\tau_{\varnothing}}+(-1)^{1} \tilde{X}_{\tau_{(2)}}$.

Now suppose we have the result for an $n \in \mathbb{N}$. Then we see that

$$
\begin{aligned}
{\left[\left[X_{1}, \ldots, X_{n}\right], X_{n+1}\right] } & =\left[X_{1}, \ldots, X_{n}\right] X_{n+1}-X_{n+1}\left[X_{1}, \ldots, X_{n}\right] \\
& =\left(\sum_{K}(-1)^{|K|} \tilde{X}_{\tau_{K}}\right) X_{n+1}-X_{n+1}\left(\sum_{K}(-1)^{|K|} \tilde{X}_{\tau_{K}}\right) \\
& =\left(\sum_{K}(-1)^{|K|} \tilde{X}_{\tau_{K}}\right) \tilde{X}_{n+1}+\tilde{X}_{n+1}\left(\sum_{K}(-1)^{|K|+1} \tilde{X}_{\tau_{K}}\right) .
\end{aligned}
$$

Now $\tau_{K} \in S_{n} \hookrightarrow S_{n+1}$, and we see that for a subsequence $K^{\prime}$ of $(2, \ldots, n+1)$ the corresponding element $\tau_{K^{\prime}} \in S_{n+1}$ leaves $n+1$ fixed if and only if $n+1 \notin K^{\prime}$ and sends $n+1$ to 1 otherwise. Thus we get

$$
\begin{aligned}
& \left(\sum_{K}(-1)^{|K|} \tilde{X}_{\tau_{K}}\right) \tilde{X}_{n+1}+\tilde{X}_{n+1}\left(\sum_{K}(-1)^{|K|+1} \tilde{X}_{\tau_{K}}\right) \\
& =\sum_{n+1 \notin K^{\prime}}(-1)^{\left|K^{\prime}\right|} \tilde{X}_{\tau_{K^{\prime}}}+\sum_{n+1 \in K^{\prime}}(-1)^{\left|K^{\prime}\right|} \tilde{X}_{\tau_{K^{\prime}}} \\
& =\sum_{K^{\prime}}(-1)^{\left|K^{\prime}\right|} \tilde{X}_{\tau_{K^{\prime}}},
\end{aligned}
$$

where all the sums are taken over subsequences $K^{\prime}$ of $(2, \ldots, n+1)$, completing the proof.

We are now in a position to prove Lemma 3.20. Let $L=F / R$, where $F$ is a free Lie ring over $X=\left\{X_{i}: i \in I\right\}$, and let $\left\{r_{\ell}: \ell \in L\right\}$ be a generating set for $R$. We have to show that for every $x \in E_{n, k} \cap \gamma_{n-1}(F)$ with $k<n$ there exists $r \in R(k)$, such that $b_{k} x+r \in E_{n, k+1}$.

Now let $F^{*}$ be the free Lie ring generated by $Y:=X \sqcup\left\{y_{\ell}: \ell \in L\right\}$ and let $\beta: F^{*} \rightarrow F$ be the homomorphism defined by $\beta\left(X_{i}\right)=X_{i}$ and $\beta\left(y_{\ell}\right)=r_{\ell}$. Then we get the following commutative diagram

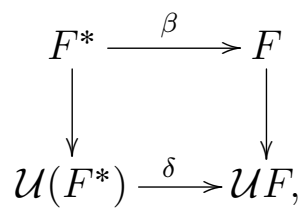

where the vertical maps are the natural homomorphisms, while $\delta$ is induced by $\beta$, i.e. $\delta\left(X_{i}\right)=X_{i}$ and $\delta\left(y_{\ell}\right)=r_{\ell}$.

Let $B=\mathcal{U} F \otimes \mathbb{Q}$ and $B^{*}=\mathcal{U} F^{*} \otimes \mathbb{Q}$ be the free Lie algebras on $X$ and $Y$ respectively over $\mathbb{Q}$. Then we have the maps $\psi$ and $\hat{\psi}$ on $B$ and $B^{*}$ defined with respect to the generators, and the map $\delta$ extends to a map from $B^{*}$ to $B$. Now 
as each $r_{\ell}$ is a Lie element of $B$ we can write it as a sum of elements of $S$ defined above. By Lemma 3.21 that means that if $W$ is the $\mathbb{Q}$-subspace of $B^{*}$ spanned by all monomials having exactly one $y_{\ell}$ and $\xi \in W$ we have

$$
\psi \delta(\xi)=\delta \psi(\xi) \text {. }
$$

Since $x \in \mathfrak{r}(k)+\mathfrak{f}^{n}$ we can write $x$ as a linear combination of monomials in $X_{i}$ and $r_{\ell}$ of degree at least $k$, each involving exactly one $r_{\ell}$, and so $x=\delta(a)$, where $a \in W$ is obtained by replacing each $r_{\ell}$ with $y_{\ell}$. Now by (3.14) and (3.15) we see that

$$
\psi\left(b_{k} a\right)=\hat{\psi}\left(b_{k} a\right)_{k}+w \in \mathcal{U} F
$$

where $w \in W$ of degree at least $k+1$, and $\hat{\psi}\left(b_{k} a\right)_{k}$ is the homogeneous component of degree $k$ of $\hat{\psi}\left(b_{k} a\right)$. By Lemma $3.22 \hat{\psi}\left(b_{k} a\right)_{k}$ is a Lie element of $\mathcal{U} F^{*}$, so we get

$$
\psi\left(b_{k} a\right)=z+w
$$

with $z \in F^{*}$ is a sum of commutators of weight $k$ in $Y$ involving exactly one $y_{\ell}$ and $w \in W$ is a sum of monomials of degree at least $k+1$. By the definition of $\delta$ we see that $\delta(w) \in \mathfrak{r}(k+1)$, and so applying $\delta$ to both sides of (3.16) and using $b_{k} a \in W$ and Lemma 3.21 we get

$$
\delta \psi\left(b_{k} a\right)=\delta(z)+\delta(w)
$$

where $\delta(z) \in R(k)$ and $\delta(w) \in \mathfrak{r}(k+1)$. On the other hand we have

$$
\delta \psi\left(b_{k} a\right)=\psi \delta\left(b_{k} a\right)=b_{k} \psi(x) .
$$

Because $x$ is homogeneous of degree $n-1$ we can use equation (3.14) again and see that $\psi(x)=\hat{\psi}(x)+f$ for an $f \in \mathfrak{f}^{n}$. But as $x \in \gamma_{n-1}(F) \subseteq F$, Lemma 3.22 gives us $\hat{\psi}(x)=x$, and putting everything together we obtain

$$
\delta(z)+\delta(w)=b_{k} x+b_{k} f,
$$

and thus

$$
b_{k} x-\delta(z)=\delta(w)-b_{k} f,
$$

and as the left hand side is obviously in $F$, the right hand side will be in $(\mathfrak{r}(k+$ $\left.1)+\mathfrak{f}^{n}\right) \cap F=E_{n, k+1}$, and as we have already seen that $\delta(z) \in R(k)$, completing the proof of Lemma 3.20 .

The Lemma enables us to finally prove

Theorem 3.25. $a_{n, k}$ divides $\prod_{\ell=k}^{n-2} b_{\ell}^{\left(\begin{array}{c}n-k-2 \\ n-\ell-2\end{array}\right)}$. In particular, for any Lie ring $L$ the exponent of $\delta_{n}(L) / \gamma_{n}(L)$ divides $\prod_{k=1}^{n-2} b_{k}^{\left(\begin{array}{c}n-2 \\ k\end{array}\right)}$.

Proof. Let $x$ and $r$ be as in Lemma 3.20 and let $y=b_{k} x+r$. Let $s=\prod_{j=k+3}^{n} a_{j, k+1}$. Then, as $y \in E_{n, k+1}$, we have $s y \in \gamma_{n}(F)+R(k+1) \subseteq \gamma_{n}(F)+R(k)$. Thus, because $r \in R(k) \subseteq \gamma_{n}(F)+R(k)$, we have $b_{k} s x \in \gamma_{n}(F)+R(k)$. So altogether

$$
a_{n, k} \mid b_{k} \prod_{j=k+3}^{n} a_{j, k+1} .
$$


Now we can bound $a_{n, k}$ by induction on $n-k$. For $n-k \leqslant 1$ we have already seen that $a_{n, k}=1$. So now let $n-k=m$ and assume that for $n-k \leqslant m-1$ we have $a_{n, k} \mid \prod_{\ell=k}^{n-2} b_{\ell}^{\left(\begin{array}{c}n-k-2 \\ n-\ell-2\end{array}\right)}$. Then because of the above formula we get

$$
a_{n, k}\left|b_{k} \prod_{j=k+3}^{n} a_{j, k+1}\right| b_{k} \prod_{j=k+3}^{n} \prod_{\ell=k+1}^{j-2} b_{\ell}^{(j-k-3)},
$$

as $j-(k+1) \leqslant n-k-1=m-1$. Now we have to count how often the factor $b_{\ell}$ appears for a fixed $\ell$. Obviously $b_{k}$ appears with exponent 1 , and only those $b_{\ell}$ with $k \leqslant \ell \leqslant n-2$ appear. Those $b_{\ell}$ appear only if $j-2 \geqslant \ell$, i.e. $j \geqslant \ell+2$, and they appear with exponent

$$
\sum_{j=\ell+2}^{n}\left(\begin{array}{c}
j-k-3 \\
j-\ell-2
\end{array}\right)=\sum_{j=\ell}^{n-2}\left(\begin{array}{c}
j-k-1 \\
j-\ell
\end{array}\right)=\left(\begin{array}{c}
n-k-2 \\
n-\ell-2
\end{array}\right)
$$

by repeated application of Pascal's rule for binomial coefficients.

By Lemma 3.18, the exponent of $\delta_{n}(L) / \gamma_{n}(L)$ divides $\prod_{j=2}^{n} a_{j, 1}$. But according to the first part of the theorem we have

$$
\prod_{j=2}^{n} a_{j, 1} \mid \prod_{j=2}^{n} \prod_{\ell=1}^{j-2} b_{\ell}^{(j-\ell-2)}
$$

For a fixed $\ell$ the exponent of $b_{\ell}$ is

$$
\sum_{j=\ell}^{n-2}\left(\begin{array}{l}
j-1 \\
j-\ell
\end{array}\right)=\left(\begin{array}{c}
n-2 \\
\ell
\end{array}\right)
$$

again by repeated application of Pascal's rule, completing the proof of Theorem 3.15

\subsection{The Dimension Problem for Metabelian Lie Rings}

As in the dimension subgroup problem, the assumption of the Lie ring $L$ to be metabelian (i.e. $[[L, L],[L, L]]=0$ ) helps simplifying calculations and thus getting stronger results. The following part is joint work with I.B.S. Passi, and is more or less identical to [36]. For notational convenience, for a Lie ring $L$, we denote by $L^{\prime}$ its derived subring $[L, L]$; thus, in particular, $L^{\prime \prime}$ denotes the second derived subring of $L$, which is trivial for metabelian Lie rings.

Theorem 3.26 ([36]). If $L$ is a metabelian Lie ring, then $2 \delta_{n}(L) \subseteq \gamma_{n}(L)$ for all $n \geqslant 1$.

By Remark 3.4, it suffices to consider only finitely generated Lie rings. So let $L$ be a finitely generated metabelian Lie ring and let $L \cong F / R$ be a pre-abelian presentation of $L$ with $F$ a free Lie ring having basis $X=\left\{X_{1}, \ldots, X_{m}\right\}$ and $R$ an ideal of $F$ generated by $e_{1} X_{1}+\xi_{1}, \ldots, e_{m} X_{m}+\xi_{m}, \xi_{m+1}, \ldots$ such that $e_{1}, \ldots, e_{m}$ 
are nonnegative integers with $e_{i} \mid e_{i+1}$ for all $i \in\{1, \ldots, m-1\}$, and $\xi_{i} \in F^{\prime}$, the derived subring of $F$. By Lemma 3.10 every finitely generated Lie ring has such a pre-abelian presentation. As $L^{\prime \prime}=0$, we have $R \supseteq F^{\prime \prime}$. Denote by $\mathfrak{r}$ the ideal of $\mathcal{U}(F)$ generated by $R$. We then have $\mathcal{U}(L) \cong \mathcal{U}(F) / \mathfrak{r}$. Furthermore, we have $\gamma_{n}(L) \cong\left(\gamma_{n}(F)+R\right) / R$ and $\delta_{n}(L) \cong\left(F \cap\left(\mathfrak{f}^{n}+\mathfrak{r}\right)\right) / R$ for all $n \geqslant 1$ by Lemma 3.7 Observe that

$$
\mathfrak{r}=\mathfrak{f} \mathfrak{r}+R
$$

thus, for any element $f \in F \cap\left(\mathfrak{f}^{n}+\mathfrak{r}\right)$, there exists an element $f^{\prime} \in$ $F \cap\left(\mathfrak{f}^{n}+\mathfrak{f r}\right)$, such that $f^{\prime} \equiv f \bmod R$.

Set $S=F^{\prime}+R$, and denote by $\mathfrak{s}$ the two-sided ideal of $\mathcal{U} F$ generated by $S$. We begin by identifying the Lie ideals

$$
M:=F \cap \mathfrak{f} \mathfrak{s} \quad \text { and } \quad F \cap\left(\mathfrak{f}^{n}+\mathfrak{f} \mathfrak{s}\right) .
$$

Lemma 3.27. With the notation as above, we have

(i) $M=\left\langle e_{i}\left[X_{i}, X_{j}\right] \mid m \geqslant i>j \geqslant 1\right\rangle+\left[F^{\prime}, S\right]$,

(ii) $[M, F] \subseteq\left[F^{\prime}, S\right]$,

(iii) $F \cap\left(\mathfrak{f}^{n}+\mathfrak{f} \mathfrak{s}\right)=\gamma_{n}(F)+M$ for all $n \geqslant 1$.

Proof. (i) It is clear that $\left[F^{\prime}, S\right]$ is contained in $F \cap \mathfrak{f s}$. Also, for $i>j$ we have $e_{j} \mid e_{i}$, and thus $e_{i}\left[X_{i}, X_{j}\right]=X_{i} e_{i} X_{j}-X_{j} e_{i} X_{i} \in F \cap \mathfrak{f s}$.

To check the reverse inclusion, recall first that $F$ acts on itself via the adjoint action:

$$
x^{y}:=\operatorname{ad}_{y}(x)=[x, y] \text { for } x, y \in F .
$$

This action extends to an action of the universal enveloping algebra $\mathcal{U} F$ on $F$, and for an associative monomial $w=X_{i_{1}} \cdots X_{i_{d}} \in \mathcal{U} F$ and $x \in F$, we have

$$
x^{w}=\left[x, X_{i_{1}}, \ldots, X_{i_{d}}\right] \text {, }
$$

where the Lie commutator is left normed.

Now suppose $v \in F \cap \mathfrak{f s}$. Then $v \in F \cap \mathfrak{f}^{2}=F^{\prime}$. Therefore,

$$
v \equiv \sum_{1 \leqslant j \leqslant m-1} \sum_{m \geqslant i>j}\left[X_{i}, X_{j}\right]^{u_{i j}} \bmod F^{\prime \prime},
$$

with $u_{i j} \in \mathcal{U} F$ involving only the variables $X_{j}, \ldots, X_{m}$. Let

$$
v_{j}:=\sum_{m \geqslant i>j}\left[X_{i}, X_{j}\right]^{u_{i j}} .
$$

For $j=1, \ldots, m-1$, define endomorphisms $\theta_{j}$ of $\mathcal{U} F$ by setting $\theta_{j}\left(X_{i}\right)=0$ for $i<j$ and $\theta_{j}\left(X_{i}\right)=X_{i}$ for $i \geqslant j$. Since both $\mathfrak{f}$ and $\mathfrak{s}$ are invariant under each $\theta_{j}$, 
successive application of the endomorphisms $\theta_{m-1}, \ldots, \theta_{1}$ shows that $v_{j} \in F \cap \mathfrak{f} \mathfrak{s}$ for $1 \leqslant j \leqslant m-1$. Write

$$
u_{i j}=n_{i j}+u_{i j}^{\prime} \text { with } n_{i j} \in \mathbb{Z}, u_{i j}^{\prime} \in \mathfrak{f}
$$

so that

$$
v_{j}=\sum_{m \geqslant i>j} n_{i j}\left[X_{i}, X_{j}\right]+\sum_{m \geqslant i>j}\left[X_{i}, X_{j}\right]^{u^{\prime}{ }^{\prime}} .
$$

Note that, modulo $\mathfrak{f} \mathfrak{s}$, the action of $\mathcal{U} F$ is just right multiplication. Thus we have, modulo $\mathfrak{f s}$,

$$
0 \equiv v_{j} \equiv \sum_{m \geqslant i>j} n_{i j}\left(X_{i} X_{j}-X_{j} X_{i}\right)+\sum_{m \geqslant i>j}\left(X_{i} X_{j}-X_{j} X_{i}\right) u_{i j}^{\prime}
$$

The right coefficient of $X_{i}, m \geqslant i>j$, in this expression is $X_{j} u_{i j}$ and must be in $\mathfrak{s}$. As $u_{i j}$ only involves the variables $X_{j}, \ldots, X_{m}$, it follows that $u_{i j}$ is divisible by $e_{j}$, so that $n_{i j}$ is divisible by $e_{j}$ and

$$
u_{i j}^{\prime}=e_{j} f_{i j}
$$

Now the right coefficient of $X_{j}$ is $\sum_{m \geqslant i>j}\left(-n_{i j} X_{i}-X_{i} u_{i j}^{\prime}\right)$ and it must also lie in $\mathfrak{s}$. Thus $e_{i} \mid n_{i j}$ for each $i>j$, as the only terms of degree one in $\mathfrak{s}$ are linear combinations of $e_{k} X_{k}, 1 \leqslant k \leqslant m$. Thus it follows that

$$
\sum_{m \geqslant i>j} X_{i} u_{i j}^{\prime} \in \mathfrak{s}
$$

Denote by $\mathfrak{a}$ the ideal of $\mathcal{U} F$ generated by $F^{\prime}$. As a acts trivially on $\left[X_{i}, X_{j}\right]$ modulo $F^{\prime \prime}$, we can change $u_{i j}^{\prime}$ modulo $\mathfrak{a}$, and thus assume, without loss of generality, that $u_{i j}^{\prime}$ and hence $f_{i j}$ is a linear combination of ordered monomials, i.e., monomials of the form $X_{k_{1}} \cdots X_{k_{t}}$ with $j \leqslant k_{1} \leqslant \ldots \leqslant k_{t} \leqslant m$. Thus we can write

$$
f_{i j}=\sum_{k=j}^{m} X_{k} g_{i j k}
$$

with $g_{i j k}$ involving only the variables $X_{k}, \ldots, X_{m}$. Thus we have

$$
\sum_{m \geqslant i>j} X_{i} u_{i j}^{\prime}=\sum_{m \geqslant i>j} \sum_{k=j}^{m} e_{j} X_{i} X_{k} g_{i j k} \in \mathfrak{s} .
$$

As $e_{j} X_{i} X_{j} g_{i j j} \in \mathfrak{s}$, we also have $\sum_{m \geqslant i>j} \sum_{k=j+1}^{m} e_{j} X_{i} X_{k} g_{i j k} \in \mathfrak{s}$. Successively applying $\theta_{j+1}, \ldots, \theta_{i-1}$ it follows inductively that $e_{k}$ divides $\sum_{m \geqslant i>j} e_{j} X_{i} X_{k} g_{i j k}$ for each $k$ with $j \leqslant k<i$. Thus we can write

$$
\sum_{m \geqslant i>j} X_{i} u_{i j}^{\prime}=\sum_{m \geqslant i>j}\left(\sum_{k=j}^{i-1} e_{k} X_{i} X_{k} g_{i j k}^{\prime}+e_{i} h_{i j}\right),
$$


where $h_{i j} \in \mathfrak{f}$ involves only the variables $X_{i}, \ldots, X_{m}$. Consequently

$$
\sum_{m \geqslant i>j}\left[X_{i}, X_{j}\right]^{u_{i j}^{\prime}}=\sum_{m \geqslant i>j} \sum_{k=j}^{i-1} e_{k}\left[X_{i}, X_{j}, X_{k}\right]^{g_{i j k}^{\prime}}+\sum_{m \geqslant i>j} e_{i}\left[X_{i}, X_{j}\right]^{h_{i j}} .
$$

Now $e_{k}\left[X_{i}, X_{j}, X_{k}\right]=\left[X_{i}, X_{j}, e_{k} X_{k}\right] \in\left[F^{\prime}, S\right]$. For $k \geqslant i$, using Jacobi identity, we have $e_{i}\left[X_{i}, X_{j}, X_{k}\right] \in\left[F^{\prime}, S\right]$. Since $h_{i j} \in \mathfrak{f}$ is a polynomial only in the variables $X_{k}$ with $k \geqslant i$, it follows that $e_{i}\left[X_{i}, X_{j}\right]^{h_{i j}} \in\left[F^{\prime}, S\right]$. Since $F^{\prime \prime}$ is contained in $\left[F^{\prime}, S\right]$, the proof of $(i)$ is complete.

(ii) follows immediately from $(i)$ in view of the Jacobi identity.

(iii) Clearly, $\gamma_{n}(F)+M \subseteq F \cap\left(\mathfrak{f}^{n}+\mathfrak{f} \mathfrak{s}\right)$ for all $n \geqslant 1$. For $n=1,2$, the reverse inclusion holds trivially. Suppose $n \geqslant 3$ and $v \in F \cap\left(\mathfrak{f}^{n}+\mathfrak{f} \mathfrak{s}\right)$. Then, proceeding as in case (i) above, we have

$$
v \equiv \sum_{m \geqslant i>j \geqslant 1}\left[X_{i}, X_{j}\right]^{u_{i j}}=\sum_{j=1}^{m-1} v_{j} \bmod \left(F^{\prime \prime}+\gamma_{n}(F)\right)
$$

and, modulo $\mathfrak{f}^{n}+\mathfrak{f} \mathfrak{s}$,

$$
0 \equiv v_{j} \equiv \sum_{m \geqslant i>j} n_{i j}\left(X_{i} X_{j}-X_{j} X_{i}\right)+\sum_{m \geqslant i>j}\left(X_{i} X_{j}-X_{j} X_{i}\right) u_{i j}^{\prime} .
$$

Therefore, the right coefficient of $X_{j}$ must be in $\varpi^{n-1}(F)+\mathfrak{s}$. Hence $n_{i j}$ must be divisible by $e_{i}$ and, modulo $\mathfrak{f}^{n-2}, u_{i j}^{\prime}$ must be divisible by $e_{j}$. Writing $u_{i j}^{\prime}=e_{j} f_{i j}+p_{i j}$ with $p_{i j} \in \mathfrak{f}^{n-2}$ and decomposing $f_{i j}$ as in the above proof of (i), we have

$$
\begin{aligned}
\sum_{m \geqslant i>j}\left[X_{i}, X_{j}\right]^{u_{i j}^{\prime}}= & \sum_{m \geqslant i>j} \sum_{k=j}^{i-1} e_{k}\left[X_{i}, X_{j}, X_{k}\right]^{g_{i j k}^{\prime}} \\
& +\sum_{m \geqslant i>j} e_{i}\left[X_{i}, X_{j}\right]^{h_{i j}}+\sum_{m \geqslant i>j}\left[X_{i}, X_{j}\right]^{p_{i j}} \in M+\gamma_{n}(F) .
\end{aligned}
$$

Hence $v \in \gamma_{n}(F)+M$.

We immediately have the following corollary.

Corollary 3.28. If $L$ is a metabelian Lie ring, then $\left[\delta_{n}(L), L\right]=\gamma_{n+1}(L)$ for all $n \geqslant 1$. In particular, the quotient $\delta_{n}(L) / \gamma_{n}(L)$ is abelian for all $n \geqslant 1$.

Proof. Since $\gamma_{n}(L) \subseteq \delta_{n}(L)$, we clearly have that $\gamma_{n+1}(L) \subseteq\left[\delta_{n}(L), L\right]$ for all $n \geqslant 1$.

For the reverse inclusion, let $L=F / R$ be a free presentation of a metabelian Lie ring $L$ and let $S=R+F^{\prime}$. Then $\left[F^{\prime}, S\right] \subseteq R$, and, by Lemma 3.27, we have

$$
\left[F \cap\left(\mathfrak{f}^{n}+\mathfrak{f r}\right), F\right] \subseteq\left[F \cap\left(\mathfrak{f}^{n}+\mathfrak{f} \mathfrak{s}\right), F\right] \subseteq \gamma_{n+1}(F)+R
$$


Hence, using equation (3.17), it follows that $\left[F \cap\left(\mathfrak{f}^{n}+\mathfrak{r}\right), F\right] \subseteq \gamma_{n+1}(F)+R$, and the Corollary is proved.

Using the characterization above, we will now prove Theorem 3.26 . Let $L$ be a finitely generated Lie ring and $L=F / R$ a pre-abelian presentation of $L$. Suppose $v \in F \cap\left(\mathfrak{f}^{n}+\mathfrak{r}\right)$. Then there exists $r \in R$ such that $w:=v+r \in F \cap\left(\mathfrak{f}^{n}+\mathfrak{f r}\right) \subseteq$ $F \cap\left(\mathfrak{f}^{n}+\mathfrak{f s}\right)$. By Lemma 3.27, we then have

$$
w \equiv \sum_{j} \sum_{m \geqslant i>j} d_{i j}\left[X_{i}, X_{j}\right]+\sum_{k} e_{k}\left[X_{k}, \eta_{k}\right] \bmod \left(\gamma_{n}(F)+F^{\prime \prime}\right)
$$

with $d_{i j} \in \mathbb{Z}$ such that $e_{i} \mid d_{i j}$, and $\eta_{k} \in F^{\prime}$. In $\mathcal{U} F$ this congruence yields

$$
w \equiv \sum_{j} \sum_{m \geqslant i>j} d_{i j}\left(X_{i} X_{j}-X_{j} X_{i}\right)+\sum_{k} e_{k} X_{k} \eta_{k} \quad \bmod \mathfrak{f}^{2} \mathfrak{s}+\mathfrak{f}^{n} .
$$

Since $w \in \mathfrak{f}^{n}+\mathfrak{f r}$, it follows that

$$
\sum_{k} X_{k}\left(y_{k}+e_{k} \eta_{k}\right) \equiv 0 \quad \bmod \left(\mathfrak{f}^{2} \mathfrak{s}+\mathfrak{f}^{n}+\mathfrak{f} \mathfrak{r}\right)
$$

where

$$
y_{k}=-\sum_{m \geqslant i>k} d_{i k} X_{i}+\sum_{1 \leqslant j<k} d_{k j} X_{j} \in F .
$$

So for every $k$ we have $y_{k}+e_{k} \eta_{k} \in \mathfrak{f} \mathfrak{s}+\mathfrak{f}^{n-1}+\mathfrak{r}$, and thus there exists $r_{k} \in \mathfrak{r} \cap F=R$ with $y_{k}+e_{k} \eta_{k}+r_{k} \in \mathfrak{f} \mathfrak{s}+\mathfrak{f}^{n-1}$. Since $\left[F^{\prime}, S\right] \subseteq S^{\prime} \subseteq R$, by Lemma 3.27 , we deduce that

$$
\left[X_{k}, y_{k}+e_{k} \eta_{k}+r_{k}\right] \in \gamma_{n}(F)+\left[F^{\prime}, S\right] \subseteq \gamma_{n}(F)+R .
$$

As $\left[X_{k}, r_{k}\right] \in R$ and $\left[X_{k}, e_{k} \eta_{k}\right]=\left[e_{k} X_{k}, \eta_{k}\right] \in R$, we conclude that

$$
\left[X_{k}, y_{k}\right] \in R+\gamma_{n}(F) \text { for every } k \text {. }
$$

Finally, the congruence 3.21 reduces, modulo $\gamma_{n}(F)+R$, to yield

$$
w \equiv \sum_{j} \sum_{m \geqslant i>j} d_{i j}\left[X_{i}, X_{j}\right]=\sum_{k} X_{k} y_{k}
$$

Recall that the map given by $u \mapsto-u$ on $F$ induces an anti-automorphism on $\mathcal{U} F$. Applying this anti-automorphism to the above equation yields

$$
-w \equiv \sum_{k} y_{k} X_{k}
$$

From the congruences $(3.22)$ and $(3.23)$, we have

$$
2 w=\sum_{k} X_{k} y_{k}-y_{k} X_{k}=\sum_{k}\left[X_{k}, y_{k}\right] \in \gamma_{n}(F)+R,
$$

and the proof is complete. 


\subsection{Dimension Quotients of Lie rings}

In this section we will try to find the structure of $\varpi^{n}(L) / \varpi^{m}(L)$ for a given Lie ring $L$ and $n, m \in \mathbb{N}$ with $n \leqslant m$. Knowing these structures can help identifying Lie dimension subrings, as the kernel of the natural map $\gamma_{n}(L) / \gamma_{m}(L) \rightarrow \varpi^{n}(L) / \varpi^{m}(L)$ is precisely $\left(\gamma_{n}(L) \cap \varpi^{m}(L)\right) / \gamma_{m}(L)$.

For a $\mathbb{Z}$-module $A$, let $\operatorname{Sym}^{n}(A)$ denote its $n$-th symmetric power, that is

$$
\operatorname{Sym}^{n}(A)=A^{\otimes n} /\left\langle x_{1} \otimes \cdots \otimes x_{n}-x_{\sigma(1)} \otimes \cdots \otimes x_{\sigma(n)}: x_{i} \in A, \sigma \in S_{n}\right\rangle .
$$

We denote by $x_{1} \vee \ldots \vee x_{n}$ the equivalence class of $x_{1} \otimes \cdots \otimes x_{n}$ in $\operatorname{Sym}^{n}(A)$. For a Lie ring $L$ define the abelian group

$$
W_{n}(L)=\bigoplus_{\left(a_{1}, \ldots, a_{n}\right)} \bigotimes_{i=1}^{n} \operatorname{Sym}^{a_{i}}\left(\gamma_{i}(L) / \gamma_{i+1}(L)\right)
$$

where the direct sum is taken over all sequences $\left(a_{1}, \ldots, a_{n}\right)$ of non-negative integers with $\sum_{i} i a_{i}=n$. Let $G$ denote a finitely generated free group on a set $X=\left\{X_{1}, \ldots, X_{m}\right\}$, and let $\gamma_{n}(G)$ denote the $n$-th term of its lower central series. Let $F$ be the free Lie ring on $X$. Then by [30], we have

$$
F \simeq \bigoplus_{i \geqslant 1} \gamma_{i}(G) / \gamma_{i+1}(G)
$$

where the Lie structure on the right hand side is given as in Example 2.16. Moreover, the $i$-th homogeneous component of a free Lie ring $F$ is precisely given by $\gamma_{i}(G) / \gamma_{i+1}(G)$.

Denote now by $\mathbb{Z} G$ the integral group ring of the free group $G$, and by $\mathfrak{g}$ its augmentation ideal, i.e. the ideal spanned by all $g-1$ for $g \in G$. Then $\mathbb{Z} G / \mathfrak{g}^{n}$ is isomorphic to the free truncated associative algebra on $X$, where all polynomials of degree at least $n$ are equated to zero. Thus we have

$$
\mathbb{Z} G / \mathfrak{g}^{n} \simeq \mathcal{U} F / \mathfrak{f}^{n}
$$

for every $n \in \mathbb{N}$. As this isomorphism preserves degrees, we also get

$$
\mathfrak{g}^{n} / \mathfrak{g}^{m} \simeq \mathfrak{f}^{n} / \mathfrak{f}^{m}
$$

for every $m \geqslant n$. In [40], Sandling and Tahara proved that for a free group $G$ we have

$$
\mathfrak{g}^{n} / \mathfrak{g}^{n+1} \simeq W_{n}(G) .
$$

Thus applying the isomorphisms given above yields

Theorem 3.29. For a free Lie ring $F$ we have

$$
\mathfrak{f}^{n} / \mathfrak{f}^{n+1} \simeq W_{n}(F)
$$

for every $n \in \mathbb{N}$. 
It follows by induction that for $n \leqslant m$ we have

$$
\mathfrak{f}^{n} / \mathfrak{f}^{m} \simeq \bigoplus_{i=n}^{m-1} W_{i}(F) .
$$

Now for an arbitrary Lie ring $L$ we get a result similar to the group case.

Proposition 3.30. For every Lie ring L, there is an isomorphism

$$
L / \gamma_{2}(L) \simeq \varpi(L) / \varpi^{2}(L)=W_{1}(L) .
$$

Proof. In the case that $L=F$ is a free Lie ring generated by a set $X, F / \gamma_{2}(F)$ is isomorphic to the free abelian group on $X$, and so is $\mathfrak{f} / \mathfrak{f}^{2}$.

Now take an arbitrary Lie ring $L=F / R$. Then because $\varpi^{2}(L) \simeq\left(\mathfrak{f}^{2}+\mathfrak{f r}+R\right) / \mathfrak{r}=$ $\left(\mathfrak{f}^{2}+R\right) / \mathfrak{r}$, we have

$$
L / \gamma_{2}(L) \simeq F /\left(\gamma_{2}(F)+R\right) \simeq \mathfrak{f} /\left(\mathfrak{f}^{2}+R\right) \simeq \varpi(L) / \varpi^{2}(L) .
$$

The following also holds, parallel to the group case.

Theorem 3.31. For a finitely generated Lie ring $L$ we have

$$
\varpi^{2}(L) / \varpi^{3}(L) \simeq \gamma_{2}(L) / \gamma_{3}(L) \oplus \operatorname{Sym}^{2}\left(L / \gamma_{2}(L)\right)=W_{2}(L) .
$$

Proof. We have a natural map $\iota: \gamma_{2}(L) / \gamma_{3}(L) \rightarrow \varpi^{2}(L) / \varpi^{3}(L), x+\gamma_{3}(L) \mapsto$ $x+\varpi^{3}(L)$. As for general Lie rings the equality $\gamma_{3}(L)=L \cap \varpi^{3}(L)$ holds, the map is a monomorphism of abelian groups. Now we need to prove that its cokernel is isomorphic to $\operatorname{Sym}^{2}\left(L / \gamma_{2}(L)\right)$.

Let $X=\left\{x_{1}, \ldots, x_{m}\right\}$ be a set of generators for $L$. By the Poincaré-Birkhoff-WittTheorem $\varpi^{2}(L) / \varpi^{3}(L)$ is spanned by $x_{i} x_{j}$ for $i \leqslant j$ and by $\left[x_{i}, x_{j}\right]$ for $i<j$. The generators of the second type are obviously in the image of $\iota$, so modulo $\iota\left(\gamma_{2}(L) / \gamma_{3}(L)\right)$ we have $x_{i} x_{j} \equiv x_{j} x_{i}$. Now

$$
L / \gamma_{2}(L) \simeq \mathbb{Z} /\left(e_{1}\right) \oplus \cdots \oplus \mathbb{Z} /\left(e_{m}\right)
$$

with $e_{i} \mid e_{i+1}$, and $e_{i}$ is the order of $x_{i}$ in the additive group $L / \gamma_{2}(L)$. Then $\operatorname{Sym}^{2}\left(L / \gamma_{2}(L)\right)$ is an abelian group generated by all $x_{i} \vee x_{j}$ with $i \leqslant j$ with the relation $e_{i}\left(x_{i} \vee x_{j}\right)=0$, so the map coker $(\iota) \rightarrow \operatorname{Sym}^{2}\left(L / \gamma_{2}(L)\right), x_{i} x_{j} \mapsto x_{i} \vee x_{j}$ is surjective. Because $e_{i} x_{i} \in \gamma_{2}(L)$, and thus $e_{i} x_{i} x_{j} \in \varpi^{3}(L)$, the inverse map $x_{i} \vee x_{j} \mapsto x_{i} x_{j}$ is well defined, so we have an isomorphism. The short exact sequence of abelian groups

$$
0 \rightarrow \gamma_{2}(L) / \gamma_{3}(L) \rightarrow \varpi^{2}(L) / \varpi^{3}(L) \rightarrow \operatorname{Sym}^{2}\left(L / \gamma_{2}(L)\right) \rightarrow 0
$$

splits via the splitting map $x_{i} \vee x_{j} \mapsto x_{i} x_{j}$, so the theorem follows. 
Theorem 3.32. For a finitely generated Lie ring $L=F / R$ given by a pre-abelian presentation with $F=\left\langle X_{1}, \ldots, X_{m}\right\rangle$ and $R=\left\langle e_{i} X_{i}+\xi_{i}, \eta_{j}\right\rangle$ with $\xi_{i}, \eta_{j} \in \gamma_{2}(F)$ we have

$$
\varpi^{3}(L) / \varpi^{4}(L) \simeq W_{3}(L) / R_{3},
$$

where $R_{3}$ is the subgroup of $W_{3}(L)$ consisting of all elements of the form

$$
\left(0, \sum_{i} \bar{f}_{i}^{(1)} \otimes \bar{\xi}_{i}, \sum_{j} a_{j} \bar{\eta}_{j}^{(3)}\right)
$$

with $f_{i}$ a sum of monomials of degree one in the $X_{i}, a_{j} \in \mathbb{Z}$ and $\eta_{j}^{(3)}$ denoting the third homogeneous component of $\eta_{j}$, satisfying the condition

$$
\sum_{i} e_{i} f_{i}^{(1)} X_{i}+\sum_{j} a_{j} \eta_{j}^{(2)}=0
$$

where $\eta_{j}^{(2)}$ denotes the second homogeneous component of $\eta_{j}$.

Proof. We define a map

$\varphi: W_{3}(L)=\operatorname{Sym}^{3}\left(L / \gamma_{2}(L)\right) \oplus\left(L / \gamma_{2}(L) \otimes \gamma_{2}(L) / \gamma_{3}(L)\right) \oplus \gamma_{3}(L) / \gamma_{4}(L) \rightarrow \varpi^{3}(L) / \varpi^{4}(L)$

by

$$
\begin{cases}\left(\bar{X}_{i} \vee \bar{X}_{j} \vee \bar{X}_{j}, 0,0\right) & \mapsto \bar{X}_{i} \bar{X}_{j} \bar{X}_{k},(i \leqslant j \leqslant k), \\ \left(0, \bar{X}_{i} \otimes\left[\bar{X}_{j}, \bar{X}_{k}\right], 0\right) & \mapsto \bar{X}_{i}\left[\bar{X}_{j}, \bar{X}_{k}\right],(j>k), \\ \left(0,0,\left[\bar{X}_{i}, \bar{X}_{j}, \bar{X}_{k}\right]\right) & \mapsto\left[\bar{X}_{i}, \bar{X}_{j}, \bar{X}_{k}\right],(i>j \leqslant k) .\end{cases}
$$

Note that $\varpi^{3}(L) / \varpi^{4}(L) \simeq\left(\mathfrak{f}^{3}+\mathfrak{r}\right) /\left(\mathfrak{f}^{4}+\mathfrak{r}\right) \simeq \mathfrak{f}^{3} /\left(\mathfrak{f}^{4}+\left(\mathfrak{f}^{3} \cap R\right)\right)$, so the map is onto. Let $R_{3}$ be its kernel.

Now we define a map $\psi: \varpi^{3}(L) / \varpi^{4}(L) \rightarrow W_{3}(L) / R_{3}$ by defining a map on $\mathfrak{f}^{3}$ that vanishes on $\mathfrak{f}^{4}+\left(\mathfrak{f}^{3} \cap \mathfrak{r}\right)$. By the Poincaré-Birkhoff-Witt-Theorem $\mathfrak{f}^{3}$ is a free abelian group generated by $X_{i} X_{j} X_{k}$ with $i \leqslant j \leqslant k, X_{i}\left[X_{j}, X_{k}\right]$ with $j>k$ and $\left[X_{i}, X_{j}, X_{k}\right]$ with $i>j \leqslant k$ and terms of degree at least four, i.e. $\mathfrak{f}^{4}$. We define

$$
\begin{array}{r}
\psi\left(X_{i} X_{j} X_{k}\right)=\left(\bar{X}_{i} \vee \bar{X}_{j} \vee \bar{X}_{j}, 0,0\right)+R_{3}(i \leqslant j \leqslant k), \\
\psi\left(X_{i}\left[X_{j}, X_{k}\right]\right)=\left(0, \bar{X}_{i} \otimes\left[\bar{X}_{j}, \bar{X}_{k}\right], 0\right)+R_{3}(j>k), \\
\psi\left(\left[X_{i}, X_{j}, X_{k}\right]\right)=\left(0,0,\left[\bar{X}_{i}, \bar{X}_{j}, \bar{X}_{k}\right]\right)(i>j \leqslant k)+R_{3}, \\
\psi\left(\mathfrak{f}^{4}\right)=0 .
\end{array}
$$

Then $\psi$ is clearly onto and vanishes in degree at least 4 . We now calculate its image on $\mathfrak{f}^{3} \cap \mathfrak{r}$. Every element $r \in \mathfrak{r}$ can be written as

$$
r=\sum_{i} f_{i}\left(e_{i} X_{i}+\xi_{i}\right)+\sum_{j} g_{j} \eta_{j}
$$

with $f_{i}, g_{j} \in \mathcal{U} F$. Denote by $f_{i}^{(k)}, g_{j}^{(k)}, \xi_{i}^{(k)}, \eta_{j}^{(k)}$ the homogeneous component of degree $k$ of $f_{i}, g_{j}, \xi_{i}, \eta_{j}$ respectively. As $\xi_{i}, \eta_{j} \in \gamma_{2}(F)$ we have $\xi_{i}^{(1)}=\eta_{j}^{(1)}=0$. Now 
if we write $r$ as above and assume $r \in \mathfrak{f}^{3}$ Then the homogeneous component of degree 1 and 2 of $r$ must be zero. It follows that $f_{i}^{(0)}=0$ for all $i$. For the degree 2 component we get

$$
\sum_{i} f_{i}^{(1)} e_{i} X_{i}+\sum_{j} g_{j}^{(0)} \eta_{j}^{(2)}=0
$$

Under this condition we have to see the image of the third degree component of $r$. It will be given by

$$
\sum_{i} f_{i}^{(2)} e_{i} X_{i}+\sum_{i} f_{i}^{(1)} \xi_{i}^{(2)}+\sum_{j} g_{j}^{(1)} \eta_{j}^{(2)}+\sum_{j} g_{j}^{(0)} \eta_{j}^{(3)}
$$

As in $L$ we have $e_{i} \bar{X}_{i} \in \gamma_{2}(L)$, we see that $\bar{f}_{i}^{(2)} e_{i} \bar{X}_{i} \in \varpi^{4}(L)$, so these will map to zero. Note further that as $\eta_{j}=\sum_{k \geqslant 2} \eta_{j}^{(k)}$, we have $\eta_{j}^{(2)} \in R+\gamma_{3}(F)$. Therefore $\bar{\eta}_{j}^{(2)} \in \gamma_{3}(L)$, so

$$
\psi\left(g_{j}^{(1)} \eta_{j}^{(2)}\right)=\left(0, \bar{g}_{j}^{(1)} \otimes \bar{\eta}_{j}^{(2)}, 0\right)=(0,0,0)
$$

In total we obtain

$$
\psi(r)=\left(0, \sum_{i} \bar{f}_{i}^{(1)}\right) \otimes \bar{\xi}_{i}^{(2)}, \sum_{j} \bar{g}_{j}^{(0)} \bar{\eta}_{j}^{(3)} .
$$

As $e_{i} X_{i} \equiv-\xi_{i} \bmod R$ we see that $e_{i} X_{i} \equiv-\xi_{i}^{(2)} \bmod R+\gamma_{3}(F)$, so $e_{i} \bar{X}_{i}+\gamma_{3}(L)=$ $-\bar{\xi}_{i}^{(2)}+\gamma_{3}(L)$. Furthermore note that $\eta_{j}^{(2)}+\eta_{j}^{(3)} \in R+\gamma_{4}(F)$, so $\bar{\eta}_{j}^{(2)}+\gamma_{4}(L)=$ $-\bar{\eta}_{j}^{(3)}+\gamma_{4}(L)$. Thus

$$
\left.\varphi(\psi(r))=-\sum_{i} \bar{f}_{i}^{(1)}\right) e_{i} X_{i}-\sum_{j} \bar{g}_{j}^{(0)} \bar{\eta}_{j}^{(2)}=0
$$

by $(3.24)$. It follows that $\psi(r) \in R_{3}$, so $\psi\left(\mathfrak{f}^{4}+\left(\mathfrak{f}^{3} \cap \mathfrak{r}\right)\right)=0$, and we have an epimorphism $\bar{\psi}: \varpi^{3}(L) / \varpi^{4}(L) \rightarrow W_{3}(L) / R_{3}$. These maps are inverse to each other by definition completing the proof.

\subsection{The Simplicial Approach}

As in group rings, one can also approach the Lie Dimension Subring Problem with simplicial methods. This has been started in [3]. Let us first give some general results for the functors we need.

Denote by $\mathfrak{A} \mathfrak{b}$ the category of abelian groups, by $\mathfrak{L} \mathfrak{i} \mathfrak{e}$ the category of Lie rings and by $\mathfrak{A s s}$ the category of associative unital algebras over $\mathbb{Z}$. Then we have the following functors.

$$
\begin{aligned}
& \mathscr{L}: \mathfrak{A} \mathfrak{b} \rightarrow \mathfrak{L} \mathfrak{i} e, \quad A \mapsto \mathscr{L}(A), \\
& T: \mathfrak{A b} \rightarrow \mathfrak{A} \mathfrak{s s}, \quad A \mapsto T(A), \\
& \mathcal{U}: \mathfrak{L i e} \rightarrow \mathfrak{A s s}, \quad L \mapsto \mathcal{U}(L),
\end{aligned}
$$

where $\mathcal{U}(L)$ denotes the universal enveloping algebra of $L, T(A)$ denotes the tensor algebra of $A$ and $\mathscr{L}(A)$ denotes the Lie subring of $T(A)$ generated by $A$. The Lie 
ring $\mathscr{L}(A)$ has the universal property that every homomorphism of abelian groups $\phi: A \rightarrow L$ extends uniquely to a Lie ring homomorphism $\Phi: \mathscr{L}(A) \rightarrow L$, such that the diagram

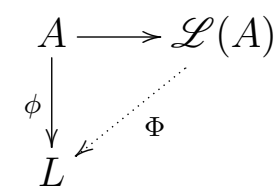

commutes. The tensor algebra has this universal property if we replace "Lie ring" by "associative algebra over $\mathbb{Z}$ ", and the universal enveloping algebra has the property if we replace "abelian group" by "Lie ring" and "Lie ring" by "associative algebra over $\mathbb{Z}$ " in the above diagram.

Proposition 3.33. We have $T=\mathcal{U} \circ \mathscr{L}$, that is $T(A)=\mathcal{U}(\mathscr{L}(A))$ for every abelian group $A$.

Proof. We show that $T(A)$ has the universal property for $\mathscr{L}(A)$ with $\iota: \mathscr{L}(A) \rightarrow$ $T(A)$ the natural embedding. So suppose $B$ is an associative algebra over $\mathbb{Z}$ and $\phi:$ $\mathscr{L}(A) \rightarrow B$ is a Lie homomorphism. As $A \subseteq \mathscr{L}(A)$, we obtain a homomorphism $\left.\phi\right|_{A}$ of abelian groups. As $B$ is an associative algebra, by the universal property of the tensor algebra, $\left.\phi\right|_{A}$ extends uniquely to a homomorphism $\Phi: T(A) \rightarrow B$.

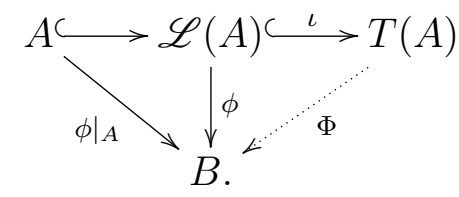

So $T(A)$ has the universal property, and therefore it is the universal enveloping algebra of $\mathscr{L}(A)$.

Definition 3.34. Let $\mathcal{C}$ be a category and $f, g: X \rightarrow Y$ two morphisms in $\mathcal{C}$. Then the coequalizer of $f$ and $g$, denoted by coeq $(f, g)$, is an object $Q$ of $\mathcal{C}$ together with a morphism $q: Y \rightarrow Q$, such that $q \circ f=q \circ g$ and for every $q^{\prime}: Y \rightarrow Q^{\prime}$ there is a unique $u: Q \rightarrow Q^{\prime}$, such that the diagram

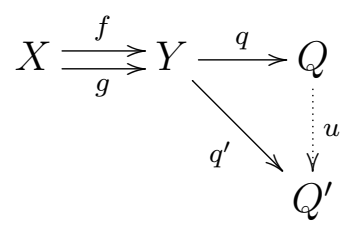

commutes.

Proposition 3.35 ([33, Proposition 5.2]). The functors $\mathscr{L}, T$ and $\mathcal{U}$ preserve coequalizers.

Proof. Suppose we have two abelian groups $A, B$ and $f, g: A \rightarrow B$. Denote by $\iota_{A}, \iota_{B}, \iota_{Q}$ the natural maps $A \rightarrow \mathscr{L}(A), B \rightarrow \mathscr{L}(B), Q \rightarrow \mathscr{L}(Q)$ respectively. Set $(Q, q)=\operatorname{coeq}(f, g)$. Then obviously $\mathscr{L}(q \circ f)=\mathscr{L}(q \circ g): \mathscr{L}(A) \rightarrow \mathscr{L}(Q)$. Suppose there is a Lie ring $L$ and a morphism $q^{\prime}: \mathscr{L}(B) \rightarrow L$ with $q^{\prime} \circ \mathscr{L}(f)=$ 
$q^{\prime} \circ \mathscr{L}(g)$. As $q^{\prime} \circ \iota_{B} \circ f=q^{\prime} \circ \mathscr{L}(f) \iota_{A}$ and $\iota_{B} \circ g=\mathscr{L}(g) \iota_{A}$, there is a unique homomorphism of abelian groups $u: Q \rightarrow L$ making the diagram commutative, as $Q=\operatorname{coeq}(f, g)$. Therefore, by the universal property of $\mathscr{L}(Q)$, there is a unique Lie ring homomorphism $\bar{u}: \mathscr{L}(Q) \rightarrow L$ such that the diagram

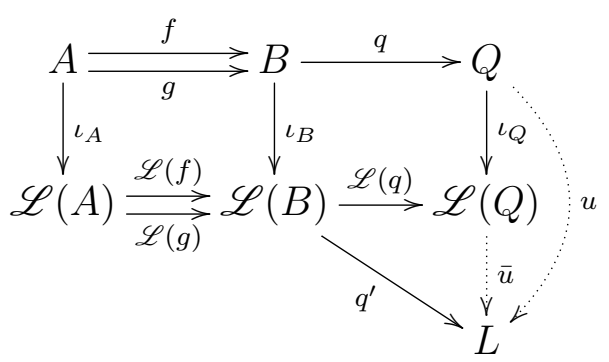

commutes. The homomorphism $\bar{u}$ is unique, because if there is $\bar{v}: \mathscr{L}(Q) \rightarrow L$ making the diagram commutative, then, as $Q$ is the coequalizer of $f$ and $g$, there is $v: Q \rightarrow L$, but then $v=u$ by uniqueness, and $\bar{v}=\bar{u}$, again by uniqueness. Hence $(\mathscr{L}(Q), \mathscr{L}(q))$ is the coequalizer of $\mathscr{L}(f)$ and $\mathscr{L}(g)$. The proofs for $T$ and $\mathcal{U}$ are analogue.

Let now $\mathbf{L}=\left\{L_{n}\right\}_{n \geqslant 0}$ be a simplicial Lie ring with Moore complex $\mathbf{M}(\mathbf{L})$ and homotopy groups $\pi_{n}(\mathbf{L})$ for $n \geqslant 0$ (see Definitions 2.57, 2.62 and 2.64). Some general results about simplicial Lie rings will be needed.

Proposition 3.36. $\pi_{0}(\mathbf{L})$ is equal to the coequalizer of the maps $d_{0}, d_{1}: L_{1} \rightarrow L_{0}$.

Proof. As Lie rings are in particular abelian groups, by Proposition 2.65 the homotopy groups are isomorphic to the homology groups of the chain complex

$$
\cdots \stackrel{\partial_{n+1}}{\longrightarrow} L_{n} \stackrel{\partial_{n}}{\longrightarrow} L_{n-1} \stackrel{\partial_{n-1}}{\longrightarrow} \ldots
$$

with $\partial_{n}=\sum_{i=0}^{n}(-1)^{i} d_{i}$

By definition of $\partial_{0}$ we have $\operatorname{ker}\left(\partial_{0}\right)=L_{0}$. Also $\partial_{1}=d_{0}-d_{1}$, so $\operatorname{im}\left(\partial_{1}\right)=\left\{d_{0}(x)-\right.$ $\left.d_{1}(x): x \in L_{1}\right\}$, and therefore

$$
\pi_{0}(\mathbf{L})=L_{0} /\left\{d_{0}(x)-d_{1}(x): x \in L_{1}\right\},
$$

which is the coequalizer of the maps $d_{0}$ and $d_{1}$.

Lemma 3.37. For a simplicial Lie ring $\mathbf{L}=\left\{L_{n}\right\}$, the abelian groups $\pi_{n}(\mathbf{L})$ are naturally endowed with a Lie ring structure, i.e. $\operatorname{im}\left(\left.d_{n+1}\right|_{M_{n+1}}\right)$ is a Lie ideal in $\operatorname{ker}\left(\left.d_{n}\right|_{M_{n}}\right)$.

Proof. Let $x \in \operatorname{im}\left(\left.d_{n+1}\right|_{M_{n+1}}\right)$, i.e. $x=d_{n+1}(y)$ with $y \in M_{n+1} \subseteq L_{n+1}$, and let $a \in \operatorname{ker}\left(\left.d_{n}\right|_{M_{n}}\right) \subseteq L_{n}$. We have to show that $[x, a] \in \operatorname{im}\left(\left.d_{n+1}\right|_{M_{n+1}}\right)$.

Note that by (2.13), $a=d_{n+1} s_{n} a$, so

$$
[x, a]=\left[d_{n+1} y, d_{n+1} s_{n} a\right]=d_{n+1}\left(\left[y, s_{n} a\right]\right),
$$

and $\left[y, s_{n} a\right] \in M_{n+1}$, as $M_{n+1}$ is a Lie ideal. 
Now the snake lemma (see e.g. [28, Chapter II, Lemma 5.2]) implies that every short exact sequence of simplicial Lie rings

$$
0 \longrightarrow \mathbf{L}_{1} \stackrel{\iota}{\longrightarrow} \mathbf{L}_{2} \stackrel{\rho}{\longrightarrow} \mathbf{L}_{3} \longrightarrow 0
$$

gives rise to a long exact sequence of homotopy groups

$$
\cdots \longrightarrow \pi_{1}\left(\mathbf{L}_{3}\right) \longrightarrow \pi_{0}\left(\mathbf{L}_{1}\right) \longrightarrow \pi_{0}\left(\mathbf{L}_{2}\right) \longrightarrow \pi_{0}\left(\mathbf{L}_{3}\right) \longrightarrow 0
$$

where the homomorphisms $\delta: \pi_{m}\left(\mathbf{L}_{3}\right) \rightarrow \pi_{m-1}\left(\mathbf{L}_{1}\right)$ are given by the snake lemma, and the others are induced by $\iota, \rho$ respectively. Thus in particular, for $n \in \mathbb{N}$ we have the endofunctors on $\mathfrak{L} \mathfrak{i} e$, the category of Lie rings,

$$
\Gamma_{n}(L)=L / \gamma_{n}(L), \quad \operatorname{gr}_{n}(L)=\gamma_{n}(L) / \gamma_{n+1}(L),
$$

which induce functors on the category $\mathfrak{s} \mathfrak{L i e}$ of simplicial Lie rings, and thus give rise to a short exact sequence

$$
0 \longrightarrow \operatorname{gr}_{n}(\mathbf{L}) \stackrel{\iota}{\longrightarrow} \Gamma_{n+1}(\mathbf{L}) \stackrel{\rho}{\longrightarrow} \Gamma_{n}(\mathbf{L}) \longrightarrow 0
$$

of simplicial Lie rings for every $n \in \mathbb{N}$. This gives a long exact sequence of homotopy groups as described above, or equivalently, an exact couple

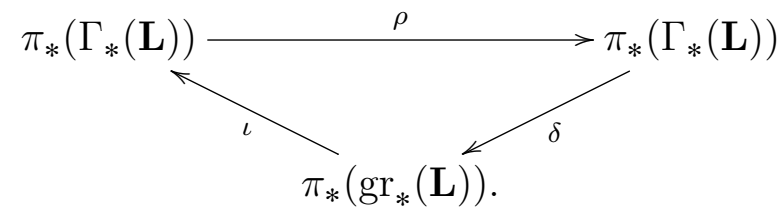

Therefore we get a spectral sequence $E$ whose first page is given by

$$
E_{p, q}^{1}=\pi_{q}\left(\operatorname{gr}_{p}(\mathbf{L})\right)
$$

and the differentials $d_{p, q}^{1}: E_{p, q}^{1} \rightarrow E_{p+1, q-1}$ are defined as follows. Take $x \in E_{p, q}^{1}=$ $\pi_{q}\left(\operatorname{gr}_{p}(\mathbf{L})\right)$, then $\iota(x) \in \pi_{q}\left(\Gamma_{p+1}(\mathbf{L})\right)$, and via the connecting homomorphism $\delta$ of the "next" long exact sequence we get $\delta(\iota(x)) \in \pi_{q-1}\left(\operatorname{gr}_{p+1}(\mathbf{L})\right)$. In pictures, we have

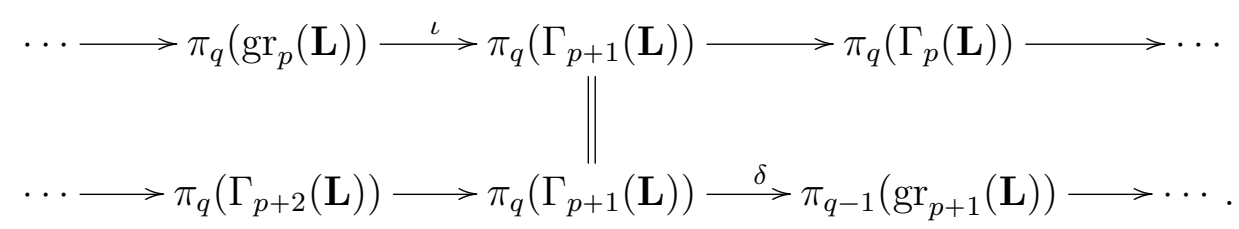

Then $d^{1} d^{1}=0$, as we follow two steps of the lower exact sequence when we compute $d^{1} d^{1}$. Therefore we can define

$$
E_{p, q}^{2}=\operatorname{ker}\left(d_{p, q}^{1}\right) / \operatorname{im}\left(d_{p-1, q+1}^{1}\right) .
$$

The differentials $d_{p, q}^{2}: E_{p, q}^{2} \rightarrow E_{p+2, q-1}$ are defined as follows. Let $[x] \in E_{p, q}^{2}$ with $x \in \operatorname{ker} d_{p, q}^{1}$. Therefore $\iota(x) \in \operatorname{ker}(\delta)$, and by exactness there is $y \in \pi_{q}\left(\Gamma_{p+2}(\mathbf{L})\right)$ 
with $\rho(y)=\iota(x)$. Then $\delta(y) \in \pi_{q-1}\left(\operatorname{gr}_{p+2}(\mathbf{L})\right)$.

In general, on the $r$-th page we have differentials $d_{p, q}^{r}: E_{p, q}^{r} \rightarrow E_{p+r, q-1}^{r}$. This can be seen from the exact couple above. We have to compute its $r$-th derived couple. This will be the following.

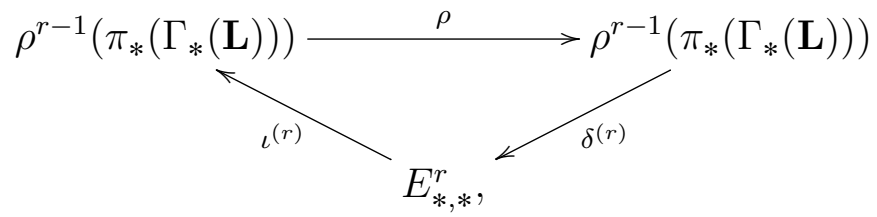

where $E_{*, *}^{r}=\operatorname{ker}\left(d^{(r-1)}\right) / \operatorname{im}\left(d^{(r-1)}\right)$ with $d^{(r-1)}=\delta^{(r-1)} \circ \iota^{(r-1)}$ the differential of $E_{*, *}^{r-1}$. The map $\iota^{(r)}$ is induced by $\iota^{(r-1)}$, i.e. $\iota^{(r)}\left(x+\operatorname{im}\left(d^{(r-1)}\right)\right)=\iota^{(r-1)}(x)+$ $\operatorname{im}\left(d^{(r-1)}\right)$ for $x \in \operatorname{ker}\left(d^{(r-1)}\right)$. The map $\delta^{(r)}$ is defined in the following way. Let $x \in \rho^{r-1}\left(\pi_{*}\left(\Gamma_{*}(\mathbf{L})\right)\right)$, then there is $y \in \rho^{r-2}\left(\pi_{*}\left(\Gamma_{*}(\mathbf{L})\right)\right)$ with $\rho(y)=x$. Then $\delta^{(r)}(x):=\delta^{(r-1)}(y)$. It can be verified that these maps are well defined and lead indeed to an exact couple. The differential on $E_{*, *}^{r}$ is then $d^{(r)}=\delta^{(r)} \circ \iota^{(r)}$. Note that $E_{p, q}^{r}$ is a subquotient of $E_{p, q}^{1}$.

We now want to compute the bidegree of $d_{p, q}^{(r)}$. It is clear that $\iota^{(r)}: E_{p, q}^{r} \rightarrow$ $\rho^{r-1}\left(\pi_{q}\left(\Gamma_{p+r}(\mathbf{L})\right)\right) \subseteq \pi_{q}\left(\Gamma_{p+1}(\mathbf{L})\right)$. As $\delta: \pi_{q}\left(\Gamma_{p+1}(\mathbf{L})\right) \rightarrow \pi_{q-1}\left(\operatorname{gr}_{p+1}(\mathbf{L})\right)$, it follows that $d_{p, q}^{(r)}=\delta^{(r)} \circ \iota^{(r)}: E_{p, q}^{r} \rightarrow E_{p+r, q-1}^{r}$, so the bidegree of $d^{(r)}$ is $(r,-1)$. Therefore, as $E_{p, q}^{r}=0$ for $q<0$ or $p \leqslant 0$, we obtain that $E_{p, 0}^{p}=E_{p, 0}^{p+1}=\cdots=E_{p, 0}^{\infty}$.

Similarly we have functors $\bar{\Gamma}_{n}, \overline{g r}{ }_{n}: \mathfrak{L i} \mathfrak{i} \rightarrow \mathfrak{A} \mathfrak{s} \mathfrak{s}$ given by

$$
\bar{\gamma}_{n}(L)=\mathcal{U}(L) / \varpi^{n}(L), \quad \overline{\mathrm{gr}}_{n}(L)=\varpi^{n}(L) / \varpi^{n+1}(L) .
$$

For a simplicial Lie ring $\mathbf{L}$, these functors give rise to a short exact sequence

$$
0 \longrightarrow \overline{g r}_{n}(\mathbf{L}) \stackrel{\bar{\iota}}{\longrightarrow} \bar{\Gamma}_{n+1}(\mathbf{L}) \stackrel{\bar{\rho}}{\longrightarrow} \bar{\Gamma}_{n}(\mathbf{L}) \longrightarrow 0
$$

for every $n \in \mathbb{N}$. This gives another exact couple

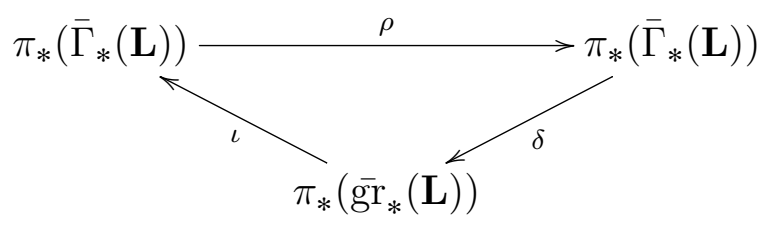

and therefore a spectral sequence $\tilde{E}$ with

$$
\tilde{E}_{p, q}^{1}=\pi_{q}\left(\overline{g r}_{p}(\mathbf{L})\right) \text {. }
$$

By the same argument as above the differentials $\tilde{d}_{p, q}^{r}$ of $\tilde{E}_{p, q}^{r}$ also have bidegree $(r,-1)$, and therefore $\tilde{E}_{p, 0}^{p}=\tilde{E}_{p, 0}^{p+1}=\cdots=E_{p, 0}^{\infty}$.

The natural embedding $i: L \rightarrow \mathcal{U}(L)$ induces a morphism of spectral sequences $i: E \rightarrow \tilde{E}$.

By definition of the functor $\mathscr{L}$, it is clear that if $F$ is a free Lie ring and $F_{\text {ab }}=F / F^{\prime}$ viewed as an abelian group, then $F \simeq \mathscr{L}\left(F_{\mathrm{ab}}\right)$. 
Theorem 3.38. Let $\mathbf{F}=\left\{F_{n}\right\}$ be a free simplicial Lie ring, i.e. a simplicial Lie ring such that each $F_{n}$ is free. Let $\mathscr{L}$ and $T$ be the functors defined in (3.25) and (3.26) and $E$ and $\tilde{E}$ the spectral sequences defined above for $\mathbf{F}$.

1. $E_{p, q}^{1}=\pi_{q}\left(\mathscr{L}_{p}\left(\mathbf{F}_{a b}\right)\right)$ and $\tilde{E}_{p, q}=\pi_{q}\left(T_{p}\left(\mathbf{F}_{a b}\right)\right)$. In particular $E_{1, q}^{1}=\tilde{E}_{1, q}^{1}=$ $\pi_{q}\left(\mathbf{F}_{a b}\right), E_{p, 0}^{1}=\mathscr{L}_{p}\left(\pi_{0}\left(\mathbf{F}_{a b}\right)\right)$ and $\tilde{E}_{p, 0}^{1}=\pi_{0}\left(T_{p}\left(\mathbf{F}_{a b}\right)\right)$.

2. $i_{*, 0}^{1}: E_{*, 0}^{1} \rightarrow \tilde{E}_{*, 0}^{1}$ is injective.

3. $E_{p, 0}^{\infty}=E_{p, 0}^{p}=\operatorname{gr}_{p}\left(\pi_{0}(\mathbf{F})\right)$ and $\tilde{E}_{p, 0}^{\infty}=\tilde{E}_{p, 0}^{p}=\overline{g r}_{p}\left(\pi_{0}(\mathbf{F})\right)$.

Proof. For a free Lie ring $F$ we see that $\operatorname{gr}_{p}(F)=\mathscr{L}_{p}\left(F_{\mathrm{ab}}\right)$ and $\overline{\mathrm{gr}}_{p}(F)=T_{p}\left(F_{\mathrm{ab}}\right)$, so the first claim follows immediately. In particular we have $\mathscr{L}_{1}\left(F_{\mathrm{ab}}\right)=T_{1}\left(F_{\mathrm{ab}}\right)=$ $F_{\mathrm{ab}}$. By Lemma $3.35 \mathscr{L}$ and $T$ preserve coequalizers, and as $\pi_{0}(\mathbf{F})=\operatorname{coeq}\left(d_{0}, d_{1}\right)$, it follows that $E_{p, 0}^{1}=\mathscr{L}_{p}\left(\pi_{0}\left(\mathbf{F}_{a b}\right)\right)$ and $\tilde{E}_{p, 0}^{1}=T_{p}\left(\pi_{0}\left(\mathbf{F}_{a b}\right)\right)$.

Note that, as a free Lie ring has the dimension property, the $\operatorname{maps}_{\operatorname{gr}}(F) \rightarrow \overline{\mathrm{gr}}_{n}(F)$ and $\Gamma_{n}(F) \rightarrow \bar{\Gamma}_{n}(F)$ induced by $i$ are monomorphisms. Therefore we have the commutative diagram of Lie ring homomorphisms

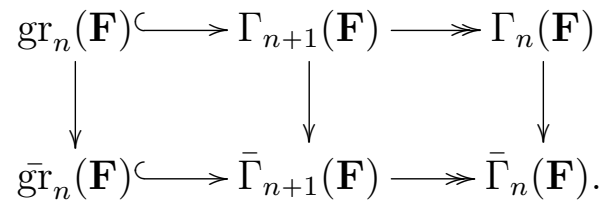

As the vertical maps are monomorphisms, the result follows.

For the last assertion we prove that $E_{p, 0}^{p}=\operatorname{ker}\left(\pi_{0}\left(\Gamma_{p+1}(\mathbf{F}) \rightarrow \pi_{0}\left(\Gamma_{p}(\mathbf{F})\right)\right)\right)$. By definition $E_{p, 0}^{p}=E_{p, 0}^{p-1} / \operatorname{im}\left(d_{1,1}^{p-1}\right)=E_{p, 0}^{p-2} /\left(\operatorname{im}\left(d_{1,1}^{p-1}\right)+\operatorname{im}\left(d_{2,1}^{p-2}\right)\right)=\cdots=\pi_{0}\left(\operatorname{gr}_{p}(\mathbf{F})\right) /\left(\operatorname{im}\left(d_{1,1}^{p-1}\right)+\right.$ $\operatorname{im}\left(d_{2,1}^{p-2}\right)+\cdots+\operatorname{im}\left(d_{p-1,1}^{1}\right)$. Note that for every $q$ we have $E_{1, q}^{1}=\pi_{q}\left(\operatorname{gr}_{1}(\mathbf{F})\right)=$ $\pi_{q}\left(\Gamma_{2}(\mathbf{F})\right)$, so the differential $d_{1, q}^{1}: E_{1, q}^{1} \rightarrow E_{2, q-1}$ is precisely the connecting homomorphism $\delta: \pi_{q}\left(\Gamma_{2}(\mathbf{F})\right) \rightarrow \pi_{q-1}\left(g_{2}(\mathbf{F})\right)$. As $E_{1,1}^{p} \subseteq E_{1,1}^{1}=\pi_{1}\left(\operatorname{gr}_{1}(\mathbf{F})\right)$, by construction of the differential, it follows that

$$
E_{p, 0}^{p}=\pi_{0}\left(\operatorname{gr}_{p}(\mathbf{F})\right) / \operatorname{im}\left(\delta: \pi_{1}\left(\Gamma_{p}(\mathbf{F})\right) \rightarrow \pi_{0}\left(\operatorname{gr}_{p}(\mathbf{F})\right)\right),
$$

and by exactness of the homotopy sequence we obtain

$$
E_{p, 0}^{p}=\pi_{0}\left(\operatorname{gr}_{p}(\mathbf{F})\right) / \operatorname{ker}(\iota) \simeq \operatorname{im}(\iota)=\operatorname{ker}\left(\pi_{0}\left(\Gamma_{p+1}(\mathbf{F})\right) \rightarrow \pi_{0}\left(\Gamma_{p}(\mathbf{F})\right)\right) .
$$

Then, as the functors $\Gamma_{k}$ also preserve coequalizers, it follows that $E_{p, 0}^{p}=\operatorname{gr}_{p}\left(\pi_{0}(\mathbf{F})\right)$. As the universal enveloping algebra functor $\mathcal{U}$ also preserves coequalizers, it follows similarly that $\tilde{E}_{p, 0}^{p}=\pi_{0}\left(\overline{\mathrm{gr}}_{p}(\mathbf{F})\right)=\overline{\mathrm{gr}}_{p}\left(\pi_{0}(\mathbf{F})\right)$.

Definition 3.39. Let $L$ be a Lie ring. A free simplicial resolution of $L$ is a simplicial Lie ring $\mathbf{F}=\left\{F_{n}: n \geqslant 0\right\}$, such that each $F_{n}$ is a free Lie ring on a set $X_{n}, s_{i}\left(X_{n}\right) \subseteq X_{n+1}$ for every $i \in\{0, \ldots, n\}$ and $\pi_{0}(\mathbf{F})=L$ and $\pi_{n}(\mathbf{F})=0$ for every $n>0$. In other words, it is a simplicial Lie ring $\mathbf{F}$ with the above conditions and an epimorphism $d_{0}: F_{0} \rightarrow L$ with $d_{0} d_{0}=d_{0} d_{1}$.

Theorem 3.40. Every Lie ring L admits a free simplicial resolution. 
Proof. Write $L=F / R$ with a free Lie ring $F$ and define $F_{0}:=F$ and denote by $X=X_{0}$ a set of free generators of $F$. Let $\left\{r_{j}: j \in J\right\}$ be a set of generators of $R$ as a Lie ideal. Set $Y=\left\{y_{j}: j \in J\right\}$ and define $X_{1}=X \sqcup Y$ and $F_{1}$ to be the free Lie ring on $Y_{1}$. The map $s_{0}: F_{0} \rightarrow F_{1}$ is the natural embedding, and

$$
\begin{array}{ccc}
d_{0}: F_{1} \rightarrow F_{0}, & d_{0}(x)=x(x \in X), & d_{0}\left(y_{j}\right)=0, \\
d_{1}: F_{1} \rightarrow F_{0}, & d_{0}(x)=x(x \in X), & d_{1}\left(y_{j}\right)=r_{j} .
\end{array}
$$

Then clearly $d_{0} s_{0}=d_{1} s_{0}=$ id, so the simplicial identities hold for these maps.

Suppose $F_{n}$ and $X_{n}$ have been defined for $n \geqslant 1$ together with face maps $d_{i}$ and degeneracy maps $s_{i}$. Then set

$$
Z_{n+1}:=\left\{\left(a_{0}, \ldots, a_{n+1}\right) \in F_{n}^{n+2}: d_{i} a_{j}=d_{j-1} a_{i} \text { for all } i<j\right\} .
$$

We can define maps

$$
d_{i}^{\prime}: Z_{n+1} \rightarrow F_{n}, \quad\left(a_{0}, \ldots, a_{n+1}\right) \mapsto a_{i}
$$

for $0 \leqslant i \leqslant n+1$ and maps

$$
s_{i}^{\prime}: F_{n} \rightarrow Z_{n+1}: a \mapsto\left(s_{i-1} d_{0} a, \ldots, s_{i-1} d_{i-1} a, a, a, s_{i} d_{i+1} a, \ldots, s_{i} d_{n} a\right)
$$

for $0 \leqslant i \leqslant n$. Then using the simplicial identities it can be shown that indeed $s_{i}^{\prime}(a) \in Z_{n+1}$, and that the simplicial identities hold for the $s_{i}^{\prime}$ and $d_{i}^{\prime}$, i.e. $d_{i} d_{j}^{\prime}=d_{j-1} d_{i}^{\prime}$ for $i<j$ etc. Now $X_{n+1}$ is given by completing $\bigcup_{i \leqslant n} s_{i}^{\prime} X_{n}$ to a set of generators of $Z_{n+1}$, and $F_{n+1}$ is the free Lie ring on $X_{n+1}$. For $x \in X_{n}$ we define $s_{i}(x)=s_{i}^{\prime}(x) \in X_{n+1}$ and extend the map to $F_{n}$. Further, for $x \in X_{n+1}$, we define $d_{i}(x)=d_{i}^{\prime}(\tilde{x})$, where $\tilde{x} \in Z_{n+1}$ corresponds to $x$, and extend the map to $F_{n+1}$.

It remains to show that $\pi_{0}(\mathbf{F})=L$ and $\pi_{n}(\mathbf{F})=0$ for $n>0$. Consider the maps $d_{0}, d_{1}: F_{1} \rightarrow F_{0}$ as defined above. Then $\pi_{0}(\mathbf{F})=F_{0} /\left\{d_{0}(a)-d_{1}(a): a \in F_{1}\right\}$. On the free generators of $F_{1}$ we have $d_{0}(x)-d_{1}(x)=x-x=0$, if $x \in X$, and $d_{0}\left(y_{j}\right)-d_{1}\left(y_{j}\right)=-r_{j}$ for $y_{j} \in Y$. Thus $R=\left\langle d_{0}(a)-d_{1}(a): a \in F_{1}\right\rangle$, and thus $L=\pi_{0}(\mathbf{F})$.

Consider now the Moore complex $\left\{M_{k}\right\}_{k \geqslant 0}$ of $\mathbf{F}$. An element $a \in M_{n}$ corresponds to an element $\tilde{a} \in \bigcap_{i \leqslant n} \operatorname{ker}\left(d_{i}^{\prime}\right)$. By definition of $d_{i}^{\prime}$, this means that $\tilde{a}=\left(0, \ldots, 0, a_{n+1}\right)$ with $d_{i}\left(a_{n+1}\right)=0$ for all $i<n$. Now the differential $M_{n+1} \rightarrow$ $M_{n}$ is given by $d_{n+1}$, so if $a \in \operatorname{ker}\left(d_{n+1}\right)$, it follows that $a_{n+1}=0$, so that $\operatorname{ker}\left(\left.d_{n+1}\right|_{M_{n+1}}\right)=0$, therefore in particular $\pi_{n+1}(\mathbf{F})=\operatorname{ker}\left(d_{n+1}\right) / \operatorname{im}\left(d_{n+2}\right)=0$ for $n \geqslant 0$.

Lemma 3.41. Let $L$ be a Lie ring and $\mathbf{F}=\left\{F_{n}: n \geqslant 0\right\}$ a free simplicial resolution of $L$. For $n \geqslant 1$ define

$$
Z_{n}(\mathbf{F}):=\left\{\left(a_{0}, \ldots, a_{n+1}\right) \in F_{n}^{n+2}: d_{i} a_{j}=d_{j-1} a_{i} \text { for all } i<j\right\} .
$$

Then the map $d^{(n)}: F_{n+1} \rightarrow Z_{n}(\mathbf{F}), a \mapsto\left(d_{0} a, \ldots, d_{n+1} a\right)$ is surjective for all $n \geqslant 1$. 
Recall Definition 2.60 of homotopy. Then we have the following theorem.

Theorem 3.42. Let $L, K$ be two Lie rings and let $\mathbf{F}=\left\{F_{n}\right\}$ and $\mathbf{G}=\left\{G_{n}\right\}$ be free simplicial resolutions of $L$ and $K$ respectively. Let $f: L \rightarrow K$ be a homomorphism. Then

1. There exists a simplicial map $\mathbf{f}: \mathbf{F} \rightarrow \mathbf{G}$ with $\pi_{0}(\mathbf{f})=f$,

2. If $\mathbf{g}: \mathbf{F} \rightarrow \mathbf{G}$ is another simplicial map with $\pi_{0}(\mathbf{g})=f$, then there exists a homotopy $h: \mathbf{f} \rightarrow \mathbf{g}$.

Proof. Let $X_{n}$ be the set of free generators of $F_{n}$ with $s_{i}\left(X_{n}\right) \subseteq X_{n+1}$. As $L \simeq$ $\pi_{0}(\mathbf{F})$, which is the coequalizer of $d_{0}, d_{1}: F_{1} \rightarrow F_{0}$, there is a natural projection $p_{L}: G_{0} \rightarrow K$. Similarly there is a natural projection $p_{K}: G_{0} \rightarrow M$. Thus for any $x \in X_{0}$ there is $y \in G_{0}$ with $p_{K}(y)=f p_{L}(x)$. Define $f_{0}(x)=y$.

Now let $n \geqslant 1$ and suppose $f_{i}$ have been defined for $i \leqslant n-1$. We want to define $f_{n}: F_{n} \rightarrow G_{n}$. Let $x \in X_{n}$. Suppose first that $x=s_{i}\left(x_{0}\right)$ with $x_{0} \in X_{n-1}$. Then we define $f_{n}(x):=s_{i} f_{n-1}\left(x_{0}\right)$. We have to show that $f_{n}$ is well defined. So assume that $x=s_{i}\left(x_{0}\right)=s_{j}\left(x_{1}\right)$ with $x_{0}, x_{1} \in X_{n-1}$. If $i=j$, then applying $d_{i}$ on both sides yields $x_{0}=x_{1}$ by (2.13). So suppose without loss of generality that $i<j$. Again we apply $d_{i}$ to obtain $x_{0}=d_{i} s_{j}\left(x_{1}\right)=s_{j-1} d_{i}\left(x_{1}\right)$ by 2.13 . Therefore $s_{i} f_{n-1}\left(x_{0}\right)=s_{i} f_{n-1} s_{j-1} d_{i}\left(x_{1}\right)=s_{i} s_{j-1} f_{n-1} d_{i}\left(x_{1}\right)=s_{j} s_{i} f_{n-1} d_{i}\left(x_{1}\right)=$ $s_{j} f_{n-1} s_{i} d_{i}\left(x_{1}\right)=s_{j} f_{n-1}\left(x_{1}\right)$. So $f_{n}$ is well defined on $\bigcup_{i} s_{i}\left(X_{n-1}\right)$. Now suppose $x \in X_{n} \backslash \bigcup_{i} s_{i}\left(X_{n-1}\right)$. Then

$$
\left(f_{n-1} d_{0}(x), \ldots, f_{n-1} d_{n}(x) \in Z_{n-1}(\mathbf{F})\right.
$$

with $Z_{n-1}(\mathbf{F})$ as in Lemma 3.41. Then by Lemma 3.41 there is $y \in G_{n}$ with $d_{i}(y)=f_{n-1} d_{i}(x)$ for all $0 \leqslant i \leqslant n$. Then define $f_{n}(x):=y$.

It remains to show that the diagrams given in Definition 2.59 commute. If $x \in$ $\bigcup_{i} s_{i}\left(X_{n-1}\right)$, then by definition $f_{n}(x)=s_{i} f_{n-1}\left(x_{0}\right)$, where $x_{0} \in X_{n-1}$ with $s_{i}\left(x_{0}\right)=$ $x$. Suppose first $j<i$. Then $d_{j} f_{n}(x)=d_{j} s_{i} f_{n-1}\left(x_{0}\right)=s_{i-1} d_{j} f_{n-1}\left(x_{0}\right)=$ $s_{i-1} f_{n-2} d_{j}\left(x_{0}\right)=f_{n-1} s_{i-1} d_{j}\left(x_{0}\right)=f_{n-1} d_{j} s_{i}\left(x_{0}\right)=f_{n-1} d_{j}(x)$, where $f_{-1}=f$ if $n=1$. Analogously we obtain the result for $j=i, j=i+1$ and for $j>i+1$ using the different cases of (2.13).

Now let $x \in X_{n-1}$. Then $s_{i}(x) \in X_{n}$, and by definition of $f_{n}$ we have $f_{n} s_{i}(x)=$ $s_{i} f_{n-1}(x)$. Thus $\mathbf{f}=\left\{f_{n}\right\}$ is a simplicial map with $\pi_{0}(\mathbf{f})=f$.

The proof of the second assertion is completely analogue to the proof for groups, which can be found in Keune [24].

By the procedure at the beginning of this section, every simplicial Lie ring gives rise to a spectral sequence. For a Lie ring $L$, we denote by $E(L)$ the spectral sequence arising from its free simplicial resolution $\mathbf{F}$. As by definition $\pi_{0}(\mathbf{F})=L$, Theorem 3.38 implies

Theorem 3.43. Let $L$ be a Lie ring and $\mathbf{F}$ its free simplicial resolution. Denote by $E, \tilde{E}$ the spectral sequences arising from $\mathbf{F}$. Then these spectral sequences have the following properties. 
1. $E_{p, 0}^{\infty}=E_{p, 0}^{p}=\operatorname{gr}_{n}(L)$ and $\tilde{E}_{p, 0}^{\infty}=\tilde{E}_{p, 0}^{p}=\overline{\operatorname{gr}}(L)$.

2. The natural embedding $i: L \rightarrow \mathcal{U} L$ induces a homomorphism $i: E \rightarrow \tilde{E}$. Furthermore $i_{*, 0}^{1}: E_{*, 0}^{1} \rightarrow \tilde{E}_{*, 0}^{1}$ is injective.

\subsection{Comparing the Results}

Let us summarize the results we have obtained for the Dimension Problem for Lie algebras, and compare them with the known results for the dimension subgroup problem, and analyze, why similarities and differences occur.

One main reason why things often work parallel is that the graded structures one obtains from a free group or a free Lie ring are isomorphic. That is, for a free group $F$ on a set $X$, the graded Lie ring $\operatorname{gr}(F)$ obtained by Example 2.16 is isomorphic to the free Lie ring on the same generating set $X$. Also, for a free Lie ring $F$, the graded Lie ring is again the free Lie ring. The same works on the level of associative algebras; For a free group $F$ and augmentation ideal $\varpi(F)$, the graded algebra $\overline{g r}(F)=\bigoplus_{n \geqslant 0} \mathfrak{f}^{n} / \mathfrak{f}^{n+1}$ is isomorphic to the free associative algebra, again exactly as in the world of Lie rings.

Another strong similarity between the theory for groups and Lie algebras is certainly Sjogren's Theorem. The constructions from the proof by Cliff and Hartley [8] could be transferred to the Lie dimension subalgebra problem without greater difficulties. Here, computations became somewhat easier, because the universal enveloping algebra of a free Lie ring is the free associative algebra, which is not (quite) the case in groups. However, modulo a power of the augmentation ideal, in both cases one gets the free truncated associative algebra.

Also, Gupta's counter examples and the simplicial constructions by Grünenfelder [14 could be translated easily. Here again, the main reason is that the graded objects of a free group and free Lie ring are isomorphic.

What worked significantly better for Lie rings was the result for metabelian Lie rings from Theorem 3.26. Here, the bilinearity of the Lie commutator proved to simplify calculations a lot, while in the group case one always has to worry about binomial coefficients appearing when simplifying the term $\left[x^{e}, y\right]$ with $e \in \mathbb{Z}$. It may still be true that metabelian groups satisfy $\delta_{n}(G)^{2} \subseteq \gamma_{n}(G)$ for all $n$, but it has not yet been proven.

What also simplifies calculations in Lie rings is that, while one can obtain a graded object from a free group or a free group ring, the free Lie ring and the free associative algebra are already graded themselves. Also, the Lie commutator in the associative algebra is homogeneous $([x, y]=x y-y x)$, while in groups the equation $[x, y]-1=x^{-1} y^{-1}((x-1)(y-1)-(y-1)(x-1))$ looks more complicated and is not homogeneous.

However, there are also some points where things work better in the theory of groups. For example, we were not able to prove Theorem 2.83 for Lie rings. Here, one reason is that the first so-called Fox subgroup $F \cap(1+\mathfrak{f r})$ for a free group $F$ and a normal subgroup $R$ can be identified as $[R, R]$ (see e.g. [4]). This is false for Lie rings by Bartholdi and Passi [3, Example 5.2]. One connected fact is that every subgroup of a free group is free (see e.g. [31, Corollary 2.9]), while a subring of a 
free Lie ring may not be free (which is the case in the aforementioned example). Recently, some progress has been made regarding the exponents of $\delta_{n}(G) / \gamma_{n}(G)$. While in all previously known examples, the exponents of the quotient were at most 2, Bartholdi and Mikhailov 2 found for every prime $p$ a group, such that some dimension quotient has $p$-torsion. For example, for $p=3$ a group is constructed whose 7 -th dimension quotient has 3 -torsion. It is not yet known whether the 5-th or 6-th dimension quotients can have 3-torsion.

Thus, some interesting problems about Lie dimension subrings are still open. We list some of them here, that may give room for future investigations.

Problem 3.44. Given a finitely generated metabelian Lie ring $L$ (or even an arbitrary finitely generated Lie ring), is there $n_{0} \in \mathbb{N}$, such that $\delta_{n}(L)=\gamma_{n}(L)$ for every $n \geqslant n_{0}$ ?

Problem 3.45. Is the quotient $\delta_{n}(L) / \gamma_{n}(L)$ always abelian? We have proven this for metabelian Lie rings (see Corollary 3.28).

Problem 3.46. Is there a Lie ring $L$ with $\gamma_{4}(L)=0$, but $\delta_{5}(L) \neq 0$ ? Or more generally, given $n \geqslant 4$, is there a number $m(n) \in \mathbb{N}$, such that $\delta_{m(n)}(L) \subseteq \gamma_{n}(L)$ for all Lie rings $L$ ? 


\section{The Ore Conjecture for Lie Algebras}

\subsection{Introduction}

We now come to a different problem in group theory, which also has an analogue in the theory of Lie algebras. As before, for a group $G$ we denote by $[G, G]$ its commutator subgroup, i.e. the group generated by all $[g, h]=g^{-1} h^{-1} g h$ with $g, h \in G$. In view of the identity $k^{-1}[g, h] k=[k, g][g, h k]$, the subgroup $[G, G]$ is a normal subgroup of $G$. Naïvely, one may think that the set of commutators is already a group. However, there is no reason why the product of two commutators is again a commutator, and indeed this is false for many groups, e.g. for the free group on two generators. This motivates the notion of the commutator width of a group $G$, which is the smallest number $n \in \mathbb{N} \cup\{\infty\}$, such that every element in $[G, G]$ can be written as product of at most $n$ commutators. If $G$ is a non-abelian finite simple group, then obviously $G=[G, G]$. This, as written above, does not imply that the commutator width of those groups is always 1 .

In 1951, Oystein Ore conjectured that every finite simple group has indeed commutator width 1 , and proved it for the alternating groups $A_{n}, n \geqslant 5$ [34]. After the conjecture had been proven for various classes of finite simple groups, in 2010 Liebeck, O'Brien, Shalev and Tiep [27] finally completed the proof of Ore's conjecture, a proof depending on the classification of finite simple groups.

The same question can be asked for finite dimensional simple Lie algebras. Is every element of these algebras a commutator? We will say that a Lie algebra $L$ has the Ore property, if every element of $[L, L]$ can be written as a commutator.

The finite dimensional Lie algebras over algebraically closed fields of characteristic zero have been fully classified (see Theorem 2.51). Those Lie algebras can be transfered into Lie algebras over fields of positive characteristic and remain simple unless the Lie algebra is $\mathfrak{s l}_{n}(k)$ with $n$ divisible by the characteristic of the field (see Theorem 2.52). We will refer to those Lie algebras as classical. It has already been proven that the classical finite dimensional simple Lie algebras have the Ore property.

Theorem 4.1 (Brown [6]). Let $L$ be a classical simple Lie algebra over a field $k$ of arbitrary characteristic and sufficiently large cardinality. Then every $x \in L$ can be expressed as a commutator.

The proof of the above result is based on two Lemmas.

Lemma 4.2 (Brown [6]). Let $L$ be a simple Lie algebra over a field $k$ of sufficiently large cardinality. Let $H$ be a Cartan Subalgebra of $L$, and let $L=H \oplus E$ as vector spaces. Then there is an $h \in H$, such that

$$
\{[h, x]: x \in L\}=E .
$$

Lemma 4.3 (Brown [6]). Let L be a simple Lie algebra over an arbitrary field, and let $E$ be as above. Then for every $x \in L$, there is an automorphism $\sigma$ of $L$, such that $\sigma(x) \in E$. 
Clearly, Theorem 4.1 follows from the two Lemmas, for if $x \in L$ is an arbitrary element, then there is an automorphism $\sigma$ of $L$, such that $\sigma(x) \in E$. Then there are $h \in H, y \in L$, such that $[h, y]=\sigma(x)$, and therefore $x=\sigma^{-1}[h, y]=$ $\left[\sigma^{-1}(h), \sigma^{-1}(y)\right]$.

Therefore, the Ore conjecture has been proven for all finite dimensional simple Lie algebras over a field of characteristic zero, as only the classical ones exist in that case.

However, over fields of positive characteristic, there are more finite dimensional simple Lie algebras than just the classical ones (see Theorem 2.55). We will make an approach to prove the Ore conjecture for one class of non-classical Lie algebras, the generalized Jacobson-Witt algebras, here. Let us first recall their definition.

Let $m \in \mathbb{N}$ and $\underline{n}=\left(n_{1}, \ldots, n_{m}\right) \in \mathbb{N}^{m}$. Let $F$ be a field of characteristic $p \geqslant 3$, and let $\mathcal{O}(m, \underline{n})$ be the Hopf algebra dual to $F\left[X_{1}, \ldots, X_{m}\right] /\left(X_{1}^{p^{n_{1}}}, \ldots, X_{m}^{p^{n_{m}}}\right)$ as in Example 2.42. Denote by $\left\{\mathbf{X}^{(a)}: a=\left(a_{1}, \ldots, a_{m}\right), 0 \leqslant a_{i}<p^{n_{i}}\right\}$ the basis of $\mathcal{O}(m, \underline{n})$ dual to the standard basis of $F\left[X_{1}, \ldots, X_{m}\right] /\left(X_{1}^{p^{n_{1}}}, \ldots, X_{m}^{p^{n_{m}}}\right)$. Then, we have

for all $a, b \in \mathbb{N}_{0}^{m}$.

$$
\mathbf{X}^{(a)} \mathbf{X}^{(b)}=\left(\begin{array}{c}
a+b \\
a
\end{array}\right) \mathbf{X}^{(a+b)}
$$

Set $e_{i}=(0, \ldots, 1, \ldots, 0)$, where the 1 occurs in the $i$-th spot. Let $\partial_{i}: \mathcal{O}(m, \underline{n}) \rightarrow$ $\mathcal{O}(m, \underline{n})$ be the $F$-linear map defined on the basis by $\partial_{i}\left(\mathbf{X}^{(a)}\right)=\mathbf{X}^{\left(a-e_{i}\right)}$ (where we set $\mathbf{X}^{\left(a-e_{i}\right)}=0$ if $\left.a_{i}=0\right)$. Then

$$
\begin{array}{r}
\partial_{i}\left(\mathbf{X}^{(a)}\right) \mathbf{X}^{(b)}+\mathbf{X}^{(b)} \partial_{i}\left(\mathbf{X}^{(a)}\right)=\left(\left(\begin{array}{c}
a+b-e_{i} \\
a-e_{i}
\end{array}\right)+\left(\begin{array}{c}
a+b-e_{i} \\
a
\end{array}\right)\right) \mathbf{X}^{\left(a+b-e_{i}\right)} \\
=\left(\begin{array}{c}
a+b \\
a
\end{array}\right) \mathbf{X}^{\left(a+b-e_{i}\right)}=\partial_{i}\left(\mathbf{X}^{(a)} \mathbf{X}^{(b)}\right) .
\end{array}
$$

Therefore the maps $\partial_{i}$, and thus every linear map of the form $\sum_{i} f_{i} \partial_{i}$ with $f_{i} \in$ $\mathcal{O}(m, \underline{n})$, are derivations of $\mathcal{O}(m, \underline{n})$. The Witt algebra $W(m, \underline{n})$ is then defined by

$$
W(m, \underline{n})=\bigoplus_{i=1}^{m} \mathcal{O}(m, \underline{n}) \partial_{i} \subseteq \operatorname{Der}(\mathcal{O}(m, \underline{n})) .
$$

As mentioned earlier, these algebras are known to be finite dimensional simple Lie algebras. The dimension of $W(m, \underline{n})$ is $m p^{|\underline{n}|}$, where $|\underline{n}|:=\sum_{i} n_{i}$.

\subsection{Generalized Jacobson-Witt Algebras in one Variable}

In this section, we will prove that the Lie algebras $W(1, \underline{n})$ have the Ore property. This will be done by purely combinatorial arguments, without using any additional assumptions on the base field. We will need a Lemma on binomial coefficients first.

Lemma 4.4. Let $p$ be an odd prime, and let $n \in \mathbb{N}$. Then, for every $0 \leqslant j<p^{n}-1$, we have

$$
\left(\begin{array}{c}
p^{n}-1 \\
j
\end{array}\right)-\left(\begin{array}{c}
p^{n}-1 \\
j+1
\end{array}\right) \not \equiv 0 \bmod p
$$


Proof. By Pascal's rule, we have $\left(\begin{array}{c}p^{n}-1 \\ j+1\end{array}\right)=\left(\begin{array}{c}p^{n} \\ j+1\end{array}\right)-\left(\begin{array}{c}p^{n}-1 \\ j\end{array}\right)$. Then $\left(\begin{array}{c}p^{n}-1 \\ j\end{array}\right)-\left(\begin{array}{c}p^{n}-1 \\ j+1\end{array}\right)=$ $\left(\begin{array}{c}p^{n} \\ j+1\end{array}\right)-2\left(\begin{array}{c}p^{n}-1 \\ j\end{array}\right)$. Now, by [46, Lemma 2.1.2], if we write $a=\sum_{k} a_{k} p^{k}$ and $b=$ $\sum_{k} b_{k} p^{k}$ with $0 \leqslant a_{k}, b_{k} \leqslant p-1$ as $p$-adic expansions, we have

$$
\left(\begin{array}{l}
a \\
b
\end{array}\right) \equiv \prod_{k}\left(\begin{array}{l}
a_{k} \\
b_{k}
\end{array}\right) \quad \bmod p .
$$

In particular, as $1 \leqslant j+1<p^{n}$, we conclude that $\left(\begin{array}{c}p^{n} \\ j+1\end{array}\right) \equiv 0 \bmod p$.

Now let $j=\sum_{k=0}^{n-1} a_{k} p^{k}$ be the $p$-adic expansion of $j$. Also, $p^{n}-1=\sum_{k=0}^{n-1}(p-1) p^{k}$ Then

$$
\left(\begin{array}{c}
p^{n}-1 \\
j
\end{array}\right)=\prod_{k=0}^{n-1}\left(\begin{array}{c}
p-1 \\
a_{k}
\end{array}\right) \not \equiv 0 \bmod p .
$$

As $p \neq 2$, we also have $2\left(\begin{array}{c}p^{n}-1 \\ j\end{array}\right) \not \equiv 0 \bmod p$.

Denote by $\partial_{X}$ the derivation of $\mathcal{O}(1, \underline{n})$ with $\partial_{X}\left(X^{(a)}\right)=X^{(a-1)}$, so that $W(1, \underline{n})=$ $\mathcal{O}(1, \underline{n}) \partial_{X}$. For $-1 \leqslant i \leqslant p^{n}-2$, set

$$
d^{(i)}=X^{(i+1)} \partial_{X} .
$$

Then the set $\left\{d^{(i)}:-1 \leqslant i \leqslant p^{n}-2\right\}$ is a basis of $W(1, \underline{n})$, whose structure constants are given by

$$
\left[d^{(i)}, d^{(j)}\right]=\left(\left(\begin{array}{c}
i+j+1 \\
j
\end{array}\right)-\left(\begin{array}{c}
i+j+1 \\
i
\end{array}\right)\right) d^{(i+j)} .
$$

Note that this makes sense, as $\left(\begin{array}{c}i+j+1 \\ j\end{array}\right)=\left(\begin{array}{c}i+j+1 \\ i\end{array}\right) \equiv 0 \bmod p$, if $i+j>p^{n}-2$. We can now prove that the Witt algebras $W(1, \underline{n})$ have the Ore property.

Theorem 4.5. Every element of $W(1, \underline{n})$ is a commutator.

Proof. Let $D=\sum_{i=-1}^{p^{n}-2} \lambda_{i+1} d^{(i)} \in W(1, \underline{n})$. Then

$$
D=\lambda_{p^{n}-1} d^{\left(p^{n}-2\right)}+[d^{(-1)}, \underbrace{\sum_{i=0}^{p^{n}-2} \lambda_{i} d^{(i)}}_{D_{1}}] .
$$

Thus, if $\lambda_{p^{n}-1}=0, D$ is a commutator.

Also, if $\lambda_{i}=0$ for all $i \neq p^{n}-1$, we can write $D=\lambda_{p^{n}-1} d^{\left(p^{n}-2\right)}=(-1 / 2) \lambda_{p^{n}-1}\left[d^{(0)}, d^{\left(p^{n}-2\right)}\right]$.

Now assume $\lambda_{p^{n}-1} \neq 0$, and $\lambda_{i} \neq 0$ for at least one $i \neq p^{n}-1$. Assume without loss of generality that $\lambda_{p^{n}-1}=1$. Let $j$ be minimal with $\lambda_{j} \neq 0$. Note that $\left[d^{(j)}, d^{(k)}\right]=0$ for $j+k>p^{n}-2$. Therefore

$$
\begin{aligned}
{\left[d^{\left(p^{n}-2-j\right)}, D_{1}\right] } & =\sum_{i=0}^{p^{n}-2} \underbrace{\lambda_{i}}_{=0, \text { if } i<j} \underbrace{\left[d^{\left(p^{n}-2-j\right)}, d^{(i)}\right]}_{=0, \text { if } i>j} \\
& =\lambda_{j}\left(\left(\begin{array}{c}
p^{n}-1 \\
j+1
\end{array}\right)-\left(\begin{array}{c}
p^{n}-1 \\
j
\end{array}\right)\right) d^{\left(p^{n}-2\right)} .
\end{aligned}
$$


Now by Lemma $4.4 \lambda_{j}\left(\left(\begin{array}{c}p^{n}-1 \\ j+1\end{array}\right)-\left(\begin{array}{c}p^{n}-1 \\ j\end{array}\right)\right) \neq 0$, so there exists $E \in W(1, \underline{n})$ with $d^{\left(p^{n}-2\right)}=\left[E, D_{1}\right]$, so altogether we conclude

$$
D=\left[E+d^{(-1)}, D_{1}\right]
$$

\subsection{The case when the field is algebraically closed}

From now on we will assume that the field $F$ is algebraically closed.

Definition 4.6. An element $D \in W(m, \underline{n})$ is called regular, if $\operatorname{dim}\left(\operatorname{ker}\left(\operatorname{ad}_{D}\right)\right)=m$.

Our strategy is as follows. Let $n \geqslant m+1$ and $\psi: F^{n} \rightarrow W(m, \underline{1})$ be an injective linear map, such that $\psi\left(\alpha_{1}, \ldots, \alpha_{n}\right)$ is regular for "almost all" $\boldsymbol{\alpha}:=\left(\alpha_{1}, \ldots, \alpha_{n}\right) \in$ $F^{n}$. Set $M(\boldsymbol{\alpha})=\operatorname{ad}_{\psi(\boldsymbol{\alpha})}$. Then, for those $\boldsymbol{\alpha}$, such that $\psi(\boldsymbol{\alpha})$ is regular, $\operatorname{ker}\left(M(\boldsymbol{\alpha})^{T}\right)$ is spanned by $\mathbf{x}_{1}(\boldsymbol{\alpha}), \ldots, \mathbf{x}_{m}(\boldsymbol{\alpha}) \in W(m, \underline{1})^{*}$. Now for a given $D \in W(m, \underline{1})$ we have $D \in M(\boldsymbol{\alpha})(W(m, \underline{1}))$ if and only if $\operatorname{ker}\left(M(\boldsymbol{\alpha})^{T}\right) \subseteq\langle D\rangle^{\perp}$. If $\psi(\boldsymbol{\alpha})$ is regular, this is equivalent to $\mathbf{x}_{i}(\boldsymbol{\alpha}) \in\langle D\rangle^{\perp}$ for all $i \in\{1, \ldots, m\}$.

Now assume that every entry of $\mathbf{x}_{i}(\boldsymbol{\alpha})$ is a homogeneous polynomial in $\alpha_{1}, \ldots, \alpha_{n}$. Then for every $i$ the equation $\left\langle D, \mathbf{x}_{i}(\boldsymbol{\alpha})\right\rangle=0$ defines a subvariety of codimension 1 in the projective space $\mathbb{P}^{n-1}(F)$. Therefore, we have $m$ varieties of codimension 1 in the $n$-1-dimensional projective space. As $n-1 \geqslant m$, these varieties intersect. If for at least one point $\boldsymbol{\alpha}$ in the intersection, the corresponding element $\psi(\boldsymbol{\alpha})$ is regular, we are done. The other cases have to be treated separately.

This method gives an alternative proof of Theorem 4.5 for $\underline{n}=\underline{1}$. Actually, even a slightly stronger statement could be obtained.

Theorem 4.7. Suppose $F$ is an algebraically closed field of characteristic $p \geqslant 3$, and let $D \in W(1, \underline{1})$. Then there are $\alpha, \beta \in F$ and $D_{1} \in W(1, \underline{1})$, such that

$$
D=\left[\alpha d^{(-1)}+\beta d^{(0)}, D_{1}\right] .
$$

Proof. By equation 4.1, we have

$$
\left[d^{(-1)}, d^{(j)}\right]=d^{(j-1)} \text { and }\left[d^{(0)}, d^{(j)}\right]=j d^{(j)} \text { for }-1 \leqslant j \leqslant p-2 .
$$

Thus writing the linear map

$$
\operatorname{ad}_{\alpha d^{(-1)}+\beta d^{(0)}}: W(1, \underline{1}) \rightarrow W(1, \underline{1}), D \mapsto\left[\alpha d^{(-1)}+\beta d^{(0)}, D\right]
$$

as a matrix $M_{\alpha, \beta}$ in the basis $\left\{d^{(i)}:-1 \leqslant i \leqslant p-2\right\}$, we see that

$$
M_{\alpha, \beta}=\left(\begin{array}{cccccc}
-\beta & \alpha & 0 & 0 & \ldots & 0 \\
0 & 0 & \alpha & 0 & \ldots & 0 \\
0 & 0 & \beta & \alpha & \ldots & 0 \\
\vdots & 0 & 0 & 2 \beta & \ldots & 0 \\
\vdots & 0 & 0 & 0 & \ddots & \vdots \\
0 & & \ldots & & -3 \beta & \alpha \\
0 & & \ldots & & & -2 \beta
\end{array}\right)
$$


This matrix has rank $p-1$ unless $\alpha=\beta=0$. We have to show that for every $D \in V:=W(1, \underline{1})$ there exist $\alpha, \beta \in F$, such that $D \in M_{\alpha, \beta}(V)$.

Consider $D=\left(v_{0}, \ldots, v_{p-1}\right) \in V$. It is known that $M_{\alpha, \beta}(V)^{\perp}=\operatorname{ker}\left(M_{\alpha, \beta}^{T}\right)$. Now $\operatorname{ker}\left(M_{\alpha, \beta}^{T}\right)$ is 1-dimensional for $(\alpha, \beta) \neq(0,0)$. It is spanned by the form

$$
f_{\alpha, \beta}:=\left(0,(p-1) ! \beta^{p-2},(p-2) ! \alpha \beta^{p-3}, \ldots, 2 ! \alpha^{p-3} \beta, \alpha^{p-2}\right) .
$$

We want to find $\alpha, \beta \in F$, such that $D \in M_{\alpha, \beta}(V)$, or equivalently, $f_{\alpha, \beta} \in\langle D\rangle^{\perp}$. This is equivalent to

$$
\sum_{i=0}^{p-1}(p-i) ! v_{i} \alpha^{i-1} \beta^{p-i-1}=0 .
$$

Now, as $F$ is algebraically closed, there are $\alpha, \beta \in F$ satisfying this polynomial equation.

Remark 4.8. The condition that $F$ is algebraically closed in Theorem 4.7 is necessary. Take for example $F=\mathbb{F}_{5}$, the field with 5 elements, and let

$$
D=\sum_{i=1}^{p-1} X^{(i)} \partial_{X}
$$

Then the polynomial equation we have to solve is

$$
-\beta^{3}+\alpha \beta^{2}+s \alpha^{2} \beta+\alpha^{3}=0 .
$$

It is clear that we have to choose $\alpha, \beta \neq 0$. As the polynomial is homogeneous, we can choose $\alpha=1$. Then $\beta$ has to satisfy

$$
-\beta^{3}+\beta^{2}+2 \beta+1=0
$$

but this polynomial equation does not have a solution in $\mathbb{F}_{5}$.

\subsection{The second Jacobson-Witt Algebra}

We will use a similar method to the above to prove that every element of the Jacobson-Witt algebra $W(2, \underline{1})$ can also be written as a commutator. In this case however, it is not as straightforward, as there are non-regular points in the intersection of the varieties we obtain. Luckily, the polynomials we get allow us to switch from $\mathbb{P}^{3}(F)$ to $\mathbb{P}^{1}(F) \times \mathbb{P}^{1}(F)$, thus avoiding most of the non-regular points. In fact, there is only one non-regular point in $\mathbb{P}^{1}(F) \times \mathbb{P}^{1}(F)$, which has to be treated separately. Here, applying an automorphism gives us the desired result.

Theorem 4.9. Every element of $W(2, \underline{1})$ over an algebraically closed field $F$ can be written as a commutator. 
Let $\boldsymbol{\alpha}=(\alpha, \beta, \gamma, \delta) \in F^{4}$, and let

$$
D_{\alpha}=\alpha \partial_{X}+\beta X \partial_{X}+\gamma \partial_{Y}+\delta Y \partial_{Y}
$$

Write again $X^{(i)}$ for $1 / i ! X^{i}$ and $Y^{(i)}$ for $1 / i ! Y^{i}$. Then

$$
\left[D_{\alpha}, X^{(i)} Y^{(j)} \partial_{X}\right]=\left(\alpha X^{(i-1)} Y^{(j)}+\gamma X^{(i)} Y^{(j-1)}+((i-1) \beta+j \delta) X^{(i)} Y^{(j)}\right) \partial_{X}
$$

and

$$
\left[D_{\alpha}, X^{(i)} Y^{(j)} \partial_{Y}\right]=\left(\alpha X^{(i-1)} Y^{(j)}+\gamma X^{(i)} Y^{(j-1)}+((j-1) \delta+i \beta) X^{(i)} Y^{(j)}\right) \partial_{Y}
$$

Denote by $I$ the $p \times p$ identity matrix, and set $M_{i}(x, y, z)=M_{x, y}+i z I$, where $M_{x, y}$ is the matrix given in the proof of Theorem 4.7. Then, with respect to the ordered basis

$$
\begin{array}{r}
X^{(0)} Y^{(0)} \partial_{X}, X^{(1)} Y^{(0)} \partial_{X}, \ldots, X^{(p-1)} Y^{(0)} \partial_{X}, X^{(0)} Y^{(1)} \partial_{X}, X^{(1)} Y^{(1)} \partial_{X}, \ldots, X^{(p-1)} Y^{(p-1)} \partial_{X}, \\
X^{(0)} Y^{(0)} \partial_{Y}, X^{(0)} Y^{(1)} \partial_{Y}, \ldots, X^{(0)} Y^{(p-1)} \partial_{Y}, X^{(1)} Y^{(0)} \partial_{Y}, X^{(1)} Y^{(1)} \partial_{Y}, \ldots, X^{(p-1)} Y^{(p-1)} \partial_{Y}
\end{array}
$$

the map $\operatorname{ad}_{D_{\alpha}}$ can be expressed by the matrix

$$
\mathbf{M}_{\boldsymbol{\alpha}}=\left(\begin{array}{cc}
M_{\boldsymbol{\alpha}, X} & 0 \\
0 & M_{\boldsymbol{\alpha}, Y}
\end{array}\right)
$$

where

$$
M_{\boldsymbol{\alpha}, X}=\left(\begin{array}{ccccc}
M_{0}(\alpha, \beta, \delta) & \gamma I & 0 & \ldots & 0 \\
0 & M_{1}(\alpha, \beta, \delta) & \gamma I & \ldots & \vdots \\
\vdots & & \ddots & & 0 \\
0 & & & M_{p-2}(\alpha, \beta, \delta) & \gamma I \\
0 & & \ldots & 0 & M_{p-1}(\alpha, \beta, \delta)
\end{array}\right)
$$

and

$$
M_{\boldsymbol{\alpha}, Y}=\left(\begin{array}{ccccc}
M_{0}(\gamma, \delta, \beta) & \alpha I & 0 & \ldots & 0 \\
0 & M_{1}(\gamma, \delta, \beta) & \alpha I & \ldots & \vdots \\
\vdots & & \ddots & & 0 \\
0 & & & M_{p-2}(\gamma, \delta, \beta) & \alpha I \\
0 & & \ldots & 0 & M_{p-1}(\gamma, \delta, \beta)
\end{array}\right)
$$

Clearly, $M_{\boldsymbol{\alpha}}$ is upper triangular, and each $M_{\boldsymbol{\alpha}, X}$ and $M_{\boldsymbol{\alpha}, Y}$ have at least one zero on the diagonal. Furthermore, if $\beta$ and $\delta$ are linearly independent over $\mathbb{F}_{p}$, there are exactly two zeros on the diagonal of $M_{\alpha}$, so the kernel of $M_{\boldsymbol{\alpha}}$ is at most 2dimensional. In fact, one easily sees that $\alpha \partial_{X}+\beta X \partial_{X}$ and $\gamma \partial_{Y}+\delta Y \partial_{Y}$ are in the kernel of $\operatorname{ad}_{D_{\boldsymbol{\alpha}}}$, so the kernel is exactly 2-dimensional in this case.

We will now determine the "bad points" $\boldsymbol{\alpha}$, for which the kernel of $M_{\boldsymbol{\alpha}}$ is more than 2-dimensional. We need a Lemma on the kernels of the $M_{i}$ first. 
Lemma 4.10. Let $z \in \mathbb{F}_{p}^{\times}$. Then $\operatorname{ker}\left(M_{i}(x, 1, z)\right) \cap \operatorname{ker}\left(M_{j}(x, 1, z)\right)=0$ if $i \neq j$.

Proof. The matrices $M_{i}(x, 1, z)$ and $M_{j}(x, 1, z)$ are upper triangular and have every element of $\mathbb{F}_{p}$ exactly once on the diagonal, with 0 on the $k$-th spot with $k \equiv$ $2-i z \bmod p$, and the $k^{\prime}$-th spot with $k^{\prime} \equiv 2-j z$ respectively. As $i \neq j$ and $z \in \mathbb{F}_{p}^{\times}$, we have $k \neq k^{\prime}$. Assume without loss of generality $k>k^{\prime}$. Let $0 \neq w=$ $\left(w_{1}, \ldots, w_{p}\right) \in \operatorname{ker}\left(M_{i}(x, 1, z)\right)$. Then $w_{\nu}=0$ for all $\nu>k$, and $w_{k} \neq 0$. But then $\left(M_{j}(x, 1, z) w\right)_{k}=(k-2+j z) w_{k} \neq 0$, and therefore $w \notin \operatorname{ker}\left(M_{j}(x, 1, z)\right)$.

Lemma 4.11. Let $\boldsymbol{\alpha}=(\alpha, \beta, \gamma, \delta) \in F^{4}$, and $\mathbf{M}_{\boldsymbol{\alpha}}$ as above. Then $\operatorname{rk}\left(\mathbf{M}_{\boldsymbol{\alpha}}\right)<$ $2 p^{2}-2$, if and only if $\boldsymbol{\alpha}=0$ or the class of $\boldsymbol{\alpha}$ in $\mathbb{P}^{3}(F)$ is one of the following:

1. $(\alpha: \beta: 0: 0)$ with $(\alpha: \beta) \in \mathbb{P}^{1}(F)$,

2. $(0: 0: \gamma: \delta)$ with $(\gamma: \delta) \in \mathbb{P}^{1}(F)$,

3. $(\alpha: 0: \gamma: 0)$ with $(\alpha: \gamma) \in \mathbb{P}^{1}(F)$,

4. or $(\alpha: 1: \gamma: j)$ with $j \in \mathbb{F}_{p}^{\times}$.

Proof. One immediately checks, that in all the above cases the kernel of $\mathbf{M}_{\boldsymbol{\alpha}}$ is more than 2-dimensional: We have $\left[(\alpha+\beta X) \partial_{X}, f(Y) \partial_{Y}\right]=0$ for all $f \in F[Y] /\left(Y^{p}\right)$, so the kernel of $\mathbf{M}_{(\alpha, \beta, 0,0)}$ is at least $p$-dimensional, similarly for $(0: 0: \gamma: \delta)$.

If $\boldsymbol{\alpha}=(\alpha, 0, \gamma, 0)$, the last and the $p^{2}$-th row of $\mathbf{M}_{\boldsymbol{\alpha}}$ are zero, while the second last row is equal to $(0, \ldots, 0, \gamma)$, which is linearly dependent to $(0, \ldots, 0, \alpha)$, the $p$-th last row. So again, the kernel is more than 2-dimensional.

Assume now, $\boldsymbol{\alpha}=(\alpha, 1, \gamma, j)$ with $j \in \mathbb{F}_{p}^{\times}$. Then, for each $i \in\{0, \ldots, p-1\}$, the matrices $M_{i}(\alpha, 1, j)$ and $M_{i}(\gamma, j, 1)$ are upper triangular with each element of $\mathbb{F}_{p}$ on the diagonal exactly once. Therefore, they are diagonalizable. One also verifies that $M_{i}(\alpha, \beta, \delta) M_{i^{\prime}}(\alpha, \beta, \delta)=M_{i^{\prime}}(\alpha, \beta, \delta) M_{i}(\alpha, \beta, \delta)$ for all $i, i^{\prime}$, so the matrices $M_{i}(\alpha, \beta, \delta)$ are simultaneously diagonalizable, so there exists $S \in \mathrm{GL}_{p}(F)$ with $S^{-1} M_{i}(\alpha, 1, j) S=D_{i}$ for all $i$, where $D_{i}$ is a diagonal matrix with each element of $\mathbb{F}_{p}$ on the diagonal exactly once, with 0 on the $k(i)$-th spot, so that $\operatorname{ker}\left(D_{i}\right)=\operatorname{span}\left(e_{k(i)}\right)$. By Lemma 4.10, it follows that $k(i) \neq k(j)$ for $i \neq j$. Similarly, there is $T \in \mathrm{GL}_{p}(F)$, such that $T^{-1} M_{i}(\gamma, j, 1) T=D_{i}^{\prime}$ for all $i$. Then conjugating $\mathbf{M}_{\alpha}$ with the $2 p^{2} \times 2 p^{2}$ block matrix $\mathbf{S}:=\operatorname{diag}(S, \ldots, S, T, \ldots, T)$ gives us a block matrix with $D_{0}, \ldots, D_{p-1}, D_{0}^{\prime}, \ldots, D_{p-1}^{\prime}$ on the diagonal, and $\gamma I, \ldots, \gamma I, 0, \alpha I, \ldots, \alpha I$ above the diagonal.

If $\gamma=0$, the kernel of $\mathbf{M}_{\boldsymbol{\alpha}}$ is obviously more than 2-dimensional. So let now $\gamma \neq 0$ and $v=\left(v_{0}, \ldots, v_{p-1}, w_{0}, \ldots, w_{p-1}\right) \in \operatorname{ker}\left(\mathbf{S}^{-1} \mathbf{M}_{\alpha} \mathbf{S}\right)$. Then $D_{p-1} v_{p-1}=0$, so $v_{p-1} \in \operatorname{span}\left(e_{k(p-1)}\right)$, so $\left(0, \ldots, 0, e_{k(p-1)}, 0, \ldots, 0\right) \in \operatorname{ker}\left(\mathbf{S}^{-1} \mathbf{M}_{\boldsymbol{\alpha}} \mathbf{S}\right)$. As $k(p-1) \neq$ $k(p-2)$, we conclude that the $k(p-1)$-th term $\mu$ on the diagonal of $D_{p-2}$ is nonzero, so $\left(0, \ldots, 0, \gamma / \mu e_{k(p-1)}, e_{k(p-1)}, 0, \ldots, 0\right)$ is another element of $\operatorname{ker}\left(\mathbf{M}_{\boldsymbol{\alpha}}\right)$, and so on.

For the converse, recall first that if $\beta$ and $\delta$ linearly independent over $\mathbb{F}_{p}$, then as mentioned above, the kernel of $M_{\alpha}$ is exactly 2-dimensional, therefore $\operatorname{rk}\left(M_{\alpha}\right)=$ $2 p^{2}-2$.

It remains to check the cases with $\beta$ and $\delta$ linearly dependent over $\mathbb{F}_{p}$, which are 
not one of the above. Let first $\beta \neq 0$ and $\delta=0$. Without loss of generality, let $\beta=1$. If $\gamma=0$, we are in case 1 , so let $\gamma \neq 0$. Then $M_{i}(\gamma, \delta, \beta)$ is invertible for all $i \neq 0$, and $M_{0}(\gamma, \delta, \beta)$ has zeroes on the diagonal and $\gamma \neq 0$ above the diagonal. Therefore, if we denote by $\mathbf{M}_{\boldsymbol{\alpha}, Y}$ the bottom right $p^{2} \times p^{2}$-block of $\mathbf{M}_{\boldsymbol{\alpha}}$, one sees that $\left(w_{0}, \ldots, w_{p-1}\right) \in \operatorname{ker}\left(\mathbf{M}_{\boldsymbol{\alpha}, Y}\right)$ if and only if $w_{1}=w_{2}=\cdots=w_{p-1}=0$ and $w_{0} \in \operatorname{ker}\left(M_{0}(\gamma, \delta, \beta)\right)$, which is one dimensional. Also, denoting by $\mathbf{M}_{\boldsymbol{\alpha}, X}$ the top left block of $\mathbf{M}_{\boldsymbol{\alpha}}$, we see that it consists of $M_{0}(\alpha, 1,0)=M_{\alpha, 1}$ on the diagonal and $\gamma I \neq 0$ above the diagonal. If $\left(v_{0}, \ldots, v_{p-1}\right) \in \operatorname{ker}\left(\mathbf{M}_{\boldsymbol{\alpha}, X}\right)$, it follows that $\left.v_{p-1} \in \operatorname{ker}\left(M_{\alpha, 1}\right)=\operatorname{span}(\alpha, 1,0, \ldots, 0)\right)$, so $v_{p-1}=\lambda(\alpha, 1,0, \ldots, 0)$ for some $\lambda \in F$. Then $M_{\alpha, 1} v_{p-2}=-\gamma \lambda(\alpha, 1,0, \ldots, 0)$. As $\gamma \neq 0$, it follows that $\lambda=0$ and $v_{p-2} \in \operatorname{ker}\left(M_{\alpha, 1}\right)$, and so on, until we get $v_{1}=\cdots=v_{p-1}=0$ and $v_{0} \in \operatorname{ker}\left(M_{\alpha, 1}\right)$. By change of variables, the case $\beta=0, \delta \neq 0$ is the same.

Lemma 4.12. For $0 \leqslant i, j \leqslant p-1$ and $\boldsymbol{\alpha}=(\alpha, \beta, \gamma, \delta) \in F^{4}$, define

$$
x_{i, j}(\boldsymbol{\alpha})=(p-i-1) !(p-j) ! \alpha^{j-1} \beta^{p-j-1} \gamma^{i} \delta^{p-i-1} .
$$

Then $\mathbf{x}(\boldsymbol{\alpha}):=\left(x_{0,0}, x_{0,1}, \ldots, x_{0, p-1}, x_{1,0}, x_{1,1}, \ldots, x_{p-1, p-1}\right) \in \operatorname{ker}\left(\mathbf{M}_{\boldsymbol{\alpha}, X}^{T}\right)$.

Proof. The $x_{i, j}(\boldsymbol{\alpha})$ satisfy the equation

$$
((j-1) \beta+i \delta) x_{i, j}+\alpha x_{i, j-1}+\gamma x_{i-1, j}=0
$$

for all $i, j$ (where $x_{*,-1}=x_{-1, *}=0$ ), which is precisely what we get in the $(i, j)$ coordinate of $\mathbf{M}_{\boldsymbol{\alpha}, X}^{T} \mathbf{x}(\boldsymbol{\alpha})$.

Let $W_{X} \oplus W_{Y}=W:=W(2, \underline{1})$ and $v=v_{X}+v_{Y} \in W$. Let $v_{X}=\left(v_{0,0}, v_{0,1}, \ldots, v_{0, p-1}\right.$, $\left.v_{1,0}, \ldots, v_{p-1 . p-1}\right)$. Then the set of $(\alpha: \beta: \gamma: \delta) \in \mathbb{P}^{3}(F)$, such that $\mathbf{x}(\boldsymbol{\alpha}) \in\left\langle v_{X}\right\rangle^{\perp}$, is given by the zero set of the homogeneous polynomial

$$
p_{v}\left(X_{0}, X_{1}, Y_{0}, Y_{1}\right):=\sum_{i, j} v_{i j}(p-j) !(p-i-1) ! X_{0}^{j-1} X_{1}^{p-j-1} Y_{0}^{i} Y_{1}^{p-i-1}
$$

of degree $2 p-3$. Similarly, if $v_{Y}=\left(w_{0,0}, \ldots, w_{p-1, p-1}\right)$, the set of $((\alpha: \beta),(\gamma: \delta))$ with $\mathbf{x} \circ \tau(\boldsymbol{\alpha}) \in\left\langle v_{Y}\right\rangle^{\perp}$ is given by the zero set of

$$
q_{v}\left(X_{0}, X_{1}, Y_{0}, Y_{1}\right)=\sum_{i, j} w_{i j}(p-i-1) !(p-j) ! X_{0}^{i} X_{1}^{p-i-1} Y_{0}^{j-1} Y_{1}^{p-j-1}
$$

of degree $2 p-3$.

Note that both these polynomials are bihomogeneous, i.e. homogeneous in $X_{0}, X_{1}$ and homogeneous in $Y_{0}, Y_{1}$. Therefore, they actually define subvarieties of $\mathbb{P}^{1}(F) \times$ $\mathbb{P}^{1}(F)$. Now, by [21, V. Example 1.4.3], two 1-dimensional subvarieties $V_{1}, V_{2}$ of $\mathbb{P}^{1} \times \mathbb{P}^{1}$ intersect, unless $V_{i}=\left\{x_{i}\right\} \times \mathbb{P}^{1}$ or $V_{i}=\mathbb{P}^{1} \times\left\{x_{i}\right\}$ with $x_{1} \neq x_{2}$. But the zero sets of $p_{v}$ and $q_{v}$ cannot be of that type, as the degrees in the $X_{i}$ and in the $Y_{i}$ are both nonzero. Therefore, there is a point $\boldsymbol{\alpha}=((\alpha: \beta),(\gamma: \delta)) \in \mathbb{P}^{1}(F) \times \mathbb{P}^{1}(F)$, such that $\mathbf{x}(\boldsymbol{\alpha}) \in\langle v\rangle^{\perp}$ and $(\mathbf{x} \circ \tau)(\boldsymbol{\alpha}) \in\langle v\rangle^{\perp}$.

If $\boldsymbol{\alpha} \neq\left((1: 0),(1: 0)\right.$, then by Lemma 4.11 , the kernel of $\mathbf{M}_{\boldsymbol{\alpha}}^{T}$ is 2-dimensional, spanned by $\mathbf{x}(\boldsymbol{\alpha})$ and $(\mathbf{x} \circ \tau)(\boldsymbol{\alpha})$. Therefore, we have $\operatorname{ker}\left(\mathbf{M}_{\boldsymbol{\alpha}}^{T}\right) \subseteq\langle v\rangle^{\perp}$, and thus 
$v \in \mathbf{M}_{\alpha}(V)$.

The only problem we are left with is what happens if the two varieties intersect only in the point $((1: 0),(1: 0))$. Note that $p_{v}(1,0,1,0)$ implies that $v_{p-1, p-1}=0$, and similarly $q_{v}(1,0,1,0)$ implies $w_{p-1, p-1}=0$. Our plan is then to apply an automorphism of $W(2,1)$, such that the zero sets of the polynomials $p_{v^{\prime}}, q_{v^{\prime}}$ defined by the image of $v$ under this automorphism intersect in other points.

Now every automorphism $\phi$ of $F[X, Y] /\left(X^{p}, Y^{p}\right)$ induces an automorphism $\bar{\phi}$ of $W(2, \underline{1})=\operatorname{Der}\left(F[X, Y] /\left(X^{p}, Y^{p}\right)\right)$ by conjugation, i.e.

$$
\bar{\phi}(D)(f)=\phi\left(D\left(\phi^{-1}(f)\right)\right)
$$

for all $D \in W(2, \underline{1})$ and $f \in F[X, Y] /\left(X^{p}, Y^{p}\right)$.

For $a, b \in F$, let $\phi_{a, b}$ be the automorphism of $F[X, Y] /\left(X^{p}, Y^{p}\right)$ with

$$
\begin{aligned}
& \phi_{a, b}(X)=X+a X^{(p-1)} Y^{(p-1)} \text { and } \\
& \phi_{a, b}(Y)=Y+b X^{(p-1)} Y^{(p-1)} .
\end{aligned}
$$

Then one checks that $\phi_{a, b}^{-1}=\phi_{-a,-b}$, and that its induced automorphism of $W(2, \underline{1})$ is given by

$$
\bar{\phi}_{a, b}\left(X^{i} Y^{j} \partial_{X}\right)=\left\{\begin{array}{l}
X^{i} Y^{j} \partial_{X}, \text { if } i+j>1 \\
\left(X+2 a X^{p-1} Y^{p-1}\right) \partial_{X}+b X^{p-1} Y^{p-1} \partial_{Y}, \text { if }(i, j)=(1,0), \\
\left(Y+b X^{p-1} Y^{p-1}\right) \partial_{X}, \text { if }(i, j)=(0,1), \\
\left(1+a X^{p-2} Y^{p-1}\right) \partial_{X}+b X^{p-2} Y^{p-1} \partial_{Y} \text { if }(i, j)=(0,0) .
\end{array}\right.
$$

Similarly one can calculate $\bar{\phi}_{a, b}\left(X^{i} Y^{j} \partial_{Y}\right)$.

Thus, if $v=\left(v_{0,0}, \ldots, v_{p-1, p-1}, w_{0,0}, \ldots, w_{p-1, p-1}\right)$ is an arbitrary element of $V$, and if $v^{\prime}=\left(v_{0,0}^{\prime}, \ldots, v_{p-1, p-1}^{\prime}, w_{0,0}^{\prime}, \ldots, w_{p-1, p-1}^{\prime}\right)$ denotes its image under $\bar{\phi}_{a b}$, then we have

$$
\begin{aligned}
v_{p-1, p-1}^{\prime} & =v_{p-1, p-1}+2 a v_{0,1}+b v_{1,0}+a w_{0,1} \text { and } \\
w_{p-1, p-1}^{\prime} & =w_{p-1, p-1}+2 b w_{0,1}+a w_{1,0}+b v_{0,1} .
\end{aligned}
$$

So if $v_{0,1} \neq 0$ or $w_{0,1} \neq 0$, one can find $a, b \in F$, such that $v_{p-1, p-1}^{\prime} \neq 0$, so that $p_{v^{\prime}}(1,0,1,0) \neq 0$, and therefore the point $((1: 0),(1: 0))$ is not in the intersection of the zero sets of $p_{v^{\prime}}$ and $q_{v^{\prime}}$. Thus $\bar{\phi}(v)$, and therefore $v$, can be written as a commutator. Finally, if $v_{0,1}=w_{0,1}=0$, the element is in the image of $D_{\alpha}$ for $\boldsymbol{\alpha}=(0,1,0, \delta)$ with $\delta \notin \mathbb{F}_{p}$, completing the proof of Theorem 4.9.

\subsection{The Higher Jacobson-Witt Algebras}

We will try and generalize this approach to the Witt algebras $W(m, \underline{1})$ with $m>2$. For $\boldsymbol{\alpha}=\left(\alpha_{1}, \beta_{1}, \alpha_{2}, \beta_{2}, \ldots, \alpha_{m}, \beta_{m}\right) \in F^{2 m}$, define

$$
D_{\boldsymbol{\alpha}}=\sum_{j=1}^{m}\left(\alpha_{j}+\beta_{j} X_{j}\right) \partial_{j} .
$$


Then, for $\mathbf{d}=\left(d_{1}, \ldots, d_{m}\right)$, one has

$$
\left[D_{\boldsymbol{\alpha}}, \mathbf{X}^{(\mathbf{d})} \partial_{i}\right]=\sum_{j=1}^{m}\left(\alpha_{j} \mathbf{X}^{\left(\mathbf{d}-e_{j}\right)}+\left(d_{j}-\delta_{i j}\right) \beta_{j} \mathbf{X}^{(\mathbf{d})}\right) \partial_{i}
$$

where as before $e_{j}=(0, \ldots, 0,1,0, \ldots, 0)$ with the 1 in the $j$-th place, and $\delta_{i j}$ denotes the Kornecker symbol. Here we set $\mathbf{X}^{\left(\mathbf{d}-e_{j}\right)}=0$ if $d_{j}=0$. So the map $\operatorname{ad}_{D_{\alpha}}$ maps the subspaces $W_{i}:=\left\{f\left(X_{1}, \ldots, X_{m}\right) \partial_{i}: f \in \mathcal{O}(m, \underline{1})\right\}$ into themselves. Let $M_{\boldsymbol{\alpha}, i}$ be the restriction of the linear map $\operatorname{ad}_{D_{\alpha}}$ to $W_{i}$.

Order the basis $\left\{\mathbf{X}^{(\mathbf{d})} \partial_{i}: \mathbf{d} \in\{0, \ldots, p-1\}^{m}\right\}$ in the following way: If $\mathbf{d} \neq \mathbf{d}^{\prime}$, let $j$ be the maximal index with $d_{j} \neq d_{j}^{\prime}$. Then $\mathbf{d}<\mathbf{d}^{\prime}$, if and only if $d_{j}<d_{j}^{\prime}$. This gives a total ordering of the basis, such that for all $i$, the matrix $M_{\boldsymbol{\alpha}, i}$ is upper triangular with respect to this ordering.

Lemma 4.13. Let $\mathcal{B}_{i}=\left\{\mathbf{X}^{(\mathbf{d})} \partial_{i}: \mathbf{d} \in\{0, \ldots, p-1\}^{m}\right\} \subseteq \mathcal{B}$ be the basis of $W_{i}$, and $\mathcal{B}_{i}^{*}=\left\{\left(\mathbf{X}^{(\mathbf{d})} \partial_{i}\right)^{*}: \mathbf{d} \in\{0, \ldots, p-1\}^{m}\right\}$ be the dual basis of $\mathcal{B}_{i}$. Let furthermore

$$
f_{\boldsymbol{\alpha}, i}=\sum_{\mathbf{d}}\left(\prod_{\ell=1}^{m}\left(p-d_{\ell}-1+\delta_{i \ell}\right) ! \alpha_{\ell}^{d_{\ell}-\delta_{i \ell}} \beta_{\ell}^{p-d_{\ell}-1}\right)\left(\mathbf{X}^{(\mathbf{d})} \partial_{i}\right)^{*} \in W_{i}^{*}
$$

Then $f_{\boldsymbol{\alpha}, i} \in \operatorname{ker}\left(M_{\boldsymbol{\alpha}, i}^{T}\right)$.

Proof. We have to show that the linear form $M_{\boldsymbol{\alpha}, i}^{T}\left(f_{\boldsymbol{\alpha}, i}\right)=f_{\boldsymbol{\alpha}, i} \circ M_{\boldsymbol{\alpha}, i}$ is the zero form on $W_{i}$, in other words

$$
\left(f_{\boldsymbol{\alpha}, i} \circ M_{\boldsymbol{\alpha}, i}\right)\left(\mathbf{X}^{(\mathbf{k})} \partial_{i}\right)=0
$$

for all $\mathbf{k} \in\{0, \ldots, p-1\}^{m}$. Indeed, by equation 4.2 ,

$$
\begin{aligned}
& \left(f_{\boldsymbol{\alpha}, i} \circ M_{\boldsymbol{\alpha}, i}\right)\left(\mathbf{X}^{(\mathbf{k})} \partial_{i}\right)=\sum_{j=1}^{m}\left(f_{\boldsymbol{\alpha}, i}\left(\alpha_{j} \mathbf{X}^{\left(\mathbf{k}-e_{j}\right)} \partial_{i}\right)+f_{\boldsymbol{\alpha}, i}\left(\left(k_{j}-\delta_{i j}\right) \beta_{j} \mathbf{X}^{(\mathbf{k})} \partial_{i}\right)\right) \\
& =\sum_{j=1}^{m} \alpha_{j} \sum_{\mathbf{d}}\left(\prod_{\ell=1}^{m}\left(p-d_{\ell}-1+\delta_{i \ell}\right) ! \alpha_{\ell}^{d_{\ell}-\delta_{i \ell}} \beta_{\ell}^{p-d_{\ell}-1}\right)\left(\mathbf{X}^{(\mathbf{d})} \partial_{i}\right)^{*}\left(\mathbf{X}^{\left(\mathbf{k}-e_{j}\right)} \partial_{i}\right) \\
& +\sum_{j=1}^{m} \beta_{j}\left(k_{j}-\delta_{i j}\right) \sum_{\mathbf{d}}\left(\prod_{\ell=1}^{m}\left(p-d_{\ell}-1+\delta_{i \ell}\right) ! \alpha_{\ell}^{d_{\ell}-\delta_{i \ell}} \beta_{\ell}^{p-d_{\ell}-1}\right)\left(\mathbf{X}^{(\mathbf{d})} \partial_{i}\right)^{*}\left(\mathbf{X}^{(\mathbf{k})} \partial_{i}\right) .
\end{aligned}
$$

Now, as by definition of the dual basis $\left(\mathbf{X}^{(\mathbf{d})} \partial_{i}\right) *\left(\mathbf{X}^{(\mathbf{k})} \partial_{i}\right)=1$ if and only if $\mathbf{d}=\mathbf{k}$, and 0 otherwise, for each $i$ only one summand in each inner sum remains.

In the first sum, for each $j$ only the summand with $\mathbf{d}=\mathbf{k}-e_{j}$, i.e. $d_{\ell}=k_{\ell}-\delta_{j \ell}$ remains, while in the second sum we only get a nonzero summand if $\mathbf{d}=\mathbf{k}$. Thus 
the above is equal to

$$
\begin{aligned}
& \sum_{j=1}^{m} \alpha_{j} \prod_{\ell=1}^{m}\left(p-k_{\ell}-1+\delta_{i \ell}+\delta_{j \ell}\right) ! \alpha_{\ell}^{k_{\ell}-\delta_{j \ell}-\delta_{i \ell}} \beta_{\ell}^{p-k_{\ell}-1+\delta_{j \ell}} \\
& +\sum_{j=1}^{m} \beta_{j}\left(k_{j}-\delta_{i j}\right) \prod_{\ell=1}^{m}\left(p-k_{\ell}-1+\delta_{i \ell}\right) ! \alpha_{\ell}^{k_{\ell}-\delta_{i \ell}} \beta_{\ell}^{p-k_{\ell}-1} \\
& =\sum_{j=1}^{m} \prod_{\ell=1}^{m}\left(p-k_{\ell}-1+\delta_{i \ell}+\delta_{j \ell}\right) ! \alpha_{\ell}^{k_{\ell}-\delta_{i \ell}} \beta_{\ell}^{p-k_{\ell}-1+\delta_{j \ell}} \\
& +\sum_{j=1}^{m}\left(k_{j}-\delta_{i j}\right) \prod_{\ell=1}^{m}\left(p-k_{\ell}-1+\delta_{i \ell}\right) ! \alpha_{\ell}^{k_{\ell}-\delta_{i \ell}} \beta_{\ell}^{p-k_{\ell}-1+\delta_{j \ell}} .
\end{aligned}
$$

For $j=i$, the summand in the first sum is

$$
\left(p-k_{i}+1\right) ! \alpha_{i}^{k_{i}-1} \beta_{i}^{p-k_{i}} \prod_{\ell \neq i}\left(p-k_{\ell}-1\right) ! \alpha_{\ell}^{k_{\ell}} \beta_{\ell}^{p-k_{\ell}-1},
$$

whereas in the second sum we get

$$
\left(k_{i}-1\right)\left(p-k_{i}\right) ! \alpha_{i}^{k_{i}-1} \beta_{i}^{p-k_{i}} \prod_{\ell \neq i}\left(p-k_{\ell}-1\right) ! \alpha_{\ell}^{k_{\ell}} \beta_{\ell}^{p-k_{\ell}-1} .
$$

Now, as in characteristic $p$, we have $\left(p-k_{i}+1\right) !=\left(p-k_{i}\right) !\left(p-k_{i}+1\right)=$ $-\left(p-k_{i}\right) !\left(k_{i}-1\right)$, the summands cancel.

Also, for $j \neq i$, in the first sum we get

$$
\left(p-k_{i}\right) ! \alpha_{i}^{k_{i}-1} \beta_{i}^{p-k_{i}-1} \prod_{\ell \neq i}\left(p-k_{\ell}-1+\delta_{j \ell}\right) ! \alpha_{\ell}^{k_{\ell}} \beta_{\ell}^{p-k_{\ell}-1+\delta_{j \ell}},
$$

while the second sum gives us

$$
k_{j}\left(p-k_{i}\right) ! \alpha_{i}^{k_{i}-1} \beta_{i}^{p-k_{i}-1} \prod_{\ell \neq i}\left(p-k_{\ell}-1\right) ! \alpha_{\ell}^{k_{\ell}} \beta_{\ell}^{p-k_{\ell}-1+\delta_{j \ell}} .
$$

As $\left(p-k_{j}\right) !=\left(p-k_{j}-1\right) !\left(p-k_{j}\right)=-k_{j}\left(p-k_{j}-1\right)$ !, those summands cancel as well.

Note that $M_{\alpha}$ is upper triangular, and that for each $i$, every linear combination of the $\beta_{j}$ with coefficients in $\mathbb{F}_{p}$ occurs on the diagonal of $M_{\boldsymbol{\alpha}, i}$ exactly once. Therefore, if the $\beta_{j}$ are linearly independent, the rank of $M_{\boldsymbol{\alpha}, i}$ is $p^{m}-1$ for every $i$, so the kernel of $M_{\boldsymbol{\alpha}, i}$ is spanned by $f_{\boldsymbol{\alpha}, i}$.

One would try to apply the same strategy as before. For a given $D=\sum_{i, \mathbf{d}} v_{i, \mathbf{d}} \mathbf{X}^{(\mathbf{d})} \partial_{i} \in$ $W(m, \underline{1})$, we define $p_{v, i} \in F\left[X_{1}, Y_{1}, \ldots, X_{m}, Y_{m}\right]$ by

$$
p_{v, i}=\sum_{\mathbf{d}} v_{i, \mathbf{d}} \prod_{\ell=1}^{m}\left(p-d_{\ell}-1+\delta_{i \ell}\right) ! X_{\ell}^{d_{\ell}-\delta_{i \ell}} Y_{\ell}^{p-d_{\ell}-1} .
$$

Each $p_{v, i}$ defines a subvariety $V_{i}$ of $\mathbb{P}^{1}(F) \times \cdots \times \mathbb{P}^{1}(F)=\left(\mathbb{P}^{1}(F)\right)^{m}$ of codimension 1. The following Lemma shows that these varieties indeed intersect. 
Lemma 4.14. Let $F$ be an algebraically closed field and let $V_{1}, \ldots, V_{m}$ be subvarieties of $\left(\mathbb{P}^{1}(F)\right)^{m}$, such that each $V_{i}$ is the zero set of a single $m$-homogeneous polynomial $p_{i} \in F\left[X_{1}, Y_{1}, \ldots, X_{m}, Y_{m}\right]$. Denote by $d_{i j}$ the bidegree of $p_{i}$ in the pair $\left(X_{j}, Y_{j}\right)$, and assume that $d_{i i}>0$ for all $i=1, \ldots, m$. Then

$$
\bigcap_{i=1}^{m} V_{i} \neq \varnothing .
$$

Proof. The second cohomology ring $H^{2}\left(\mathbb{P}^{1}(F)\right)$ is given by $\mathbb{Z}[T] /\left(T^{2}\right)$, and therefore

$$
H^{2}\left(\left(\mathbb{P}^{1}(F)\right)^{m}\right)=\left(\mathbb{Z}[T] /\left(T^{2}\right)\right)^{\otimes m} \simeq \mathbb{Z}\left[T_{1}, \ldots, T_{m}\right] /\left(T_{1}^{2}, \ldots, T_{m}^{2}\right) .
$$

Now each $V_{i}$ defines the class $\left(d_{i 1} T_{1}+\cdots+d_{i m} T_{m}\right)$ in $H^{2}\left(\left(\mathbb{P}^{1}(F)^{m}\right)\right)$, and the class of the intersection corresponds to the product of the classes. Now for $i \neq j$ the coefficient of $T_{i} T_{j}$ in the product is at least $d_{i i} d_{j j}>0$. Therefore, the intersection is not empty, as it is represented by a nonzero class.

Now the problem is, that there are more non-regular points in $\left(\mathbb{P}^{1}(F)\right)^{m}$ for $m \geqslant 3$. Indeed, all points with at least 2 coordinates equal to $(1: 0)$ are non-regular, as these points are already non-regular in a copy of $W(2, \underline{1})$ contained in $W(m, \underline{1})$. Now these are indeed the only non-regular points of $\left(\mathbb{P}^{1}(F)\right)^{m}$, as the following Lemma shows.

Lemma 4.15. Let $\boldsymbol{\alpha}=\left(\alpha_{1}, \beta_{1}, \ldots, \alpha_{m}, \beta_{m}\right) \in F^{2 m}$ such that $\beta_{1}=0, \alpha_{1} \neq 0$, and $\beta_{j}, j=2, \ldots, m$ linearly independent over $\mathbb{F}_{p}$. Then $\operatorname{rk}\left(M_{\boldsymbol{\alpha}, i}\right)=p^{m}-1$ for all $i=1, \ldots, m$.

Proof. By Equation (4.2) and because $\beta_{1}=0$, the diagonal elements of $M_{\boldsymbol{\alpha}, i}$ are given by $\sum_{j=2}^{m}\left(d_{j}-\delta_{i j}\right) \beta_{j}$. If $i=1$, then because the $\beta_{j}$ are linearly independent over $\mathbb{F}_{p}$, this is only zero, if $d_{j}=0$ for all $j \geqslant 2$. These are exactly the first $p$ entries with respect to our ordering. Now above the diagonal, we get $\alpha_{1} \neq 0$. Thus the first $p \times p$-block has rank $p-1$, while the other $p \times p$-blocks on the diagonal are invertible, thus $\operatorname{rk}\left(M_{\boldsymbol{\alpha}, 1}\right)=p^{m}-1$.

Similarly, for $i \neq 1$ the only zeroes on the diagonal of $M_{\boldsymbol{\alpha}, i}$ are for $d_{j}=\delta_{i j}$ for $j \geqslant 2$ and $d_{1}$ arbitrary. This is again a $p \times p$-block with $\alpha_{1} \neq 0$ above the diagonal, so it has rank $p-1$. All the other $p \times p$-blocks on the diagonal are invertible, so in total $\operatorname{rk}\left(M_{\boldsymbol{\alpha}, i}\right)=p^{m}-1$.

Thus, if less than 2 of the $\beta_{j}$ are nonzero, by choosing a suitable representative in every coordinate of $\left(\mathbb{P}^{1}(F)\right)^{m}$ by making the $\beta_{j}$ linearly independent over $\mathbb{F}_{p}$, the point becomes regular.

The problem now is, that the non-regular sets become larger, and therefore one has to be careful when applying an automorphism as we did for $m=2$. For example, for $m=3$, the set of non-regular points is the union of three lines, for $m=4$ even the union of six planes, etc. Therefore, the analysis becomes very hard, although one may still expect to find a suitable automorphism if the varieties happen to intersect only in non-regular points. 


\subsection{Brown's Strategy}

Let us now examine the strategy Brown used in [6] to prove the result for classical Lie algebras. As seen in the introduction to this chapter, Lemmas 4.2 and 4.3 together prove that every element of a Lie algebra is a commutator.

To apply this approach to Witt algebras, we first have to fix a Cartan subalgebra.

Lemma 4.16. Let $H$ be the span of the set $\left\{X_{i} \partial_{i}: i=1, \ldots, m\right\}$. Then $H$ is a Cartan subalgebra of $W(m, \underline{1})$.

Proof. Clearly $H$ is abelian and therefore nilpotent.

Now let $D \in W(m, \underline{1})$, such that $[D, h] \in H$ for all $h \in H$. We have to show that $D \in H$.

First of all, by the grading of $W(m, \underline{1})$, the degree of $D$ must be zero. Thus

$$
D=\sum_{i, j} a_{i j} X_{i} \partial_{j}
$$

for some $a_{i j} \in F$. Now

$$
\begin{aligned}
{\left[D, X_{k} \partial_{k}\right] } & =\sum_{i, j} a_{i j}\left[X_{i} \partial_{j}, X_{k} \partial_{k}\right] \\
& =\sum_{i=1}^{m} a_{i k} X_{i} \partial_{k}-\sum_{j=1}^{m} a_{k j} X_{k} \partial_{j} \in H
\end{aligned}
$$

for every $k \in\{1, \ldots, m\}$. From the second sum, it follows that $a_{k j}=0$ for $j \neq k$, and therefore $D \in H$.

Now with this choice of a Cartan subalgebra, we are able to prove Lemma 4.2 for the Jacobson-Witt algebras.

Lemma 4.17. Let $H$ be as above, and let $E$ be the complement of $H$ in $W(m, \underline{1})$. Then, if $|F| \geqslant p^{m}$, there is $h \in H$, such that

$$
\{[h, D]: D \in W(m, \underline{1})\}=E .
$$

Proof. As $|F| \geqslant p^{m}$ by assumption, there are $\beta_{1}, \ldots, \beta_{m} \in F$, such that $\beta_{i}$ are linearly independent over $\mathbb{F}_{p}$. Then, setting

$$
h:=\sum_{i=1}^{m} \beta_{i} X_{i} \partial_{i}
$$

we obtain

$$
\left[h, \mathbf{X}^{(\mathbf{d})} \partial_{j}\right]=\sum_{i=1}^{m}\left(d_{i}-\delta_{i j}\right) \beta_{i} \mathbf{X}^{(\mathbf{d})} \partial_{j} .
$$

Therefore, with respect to the standard basis, the matrix of $\operatorname{ad}_{h}$ is diagonal, with every linear combination of the $\beta_{i}$ with coefficients in $\mathbb{F}_{p}$ on the diagonal exactly $m$ times. Thus, the only zeroes on the diagonal are at $d_{i}=\delta_{i j}$, i.e. $\mathbf{d}=e_{i}$. Then $\operatorname{ad}_{h}(W(m, \underline{1}))=\operatorname{span}\left\{\mathbf{X}^{(\mathbf{d})} \partial_{i}: \mathbf{d} \neq e_{i}\right\}=E$. 
From the above Lemma, we can immediately bound the commutator width of $W(m, \underline{1})$ by 2 .

Corollary 4.18. If $|F| \geqslant p^{m}$, every element of $W(m, \underline{1})$ can be written as sum of at most two commutators.

Proof. Let $D \in W(m, \underline{1})$. Then we can write $D=D_{E}+D_{H}$ with $D_{E} \in E$ and $D_{H} \in H$. By Lemma 4.17, $D_{E}$ can be written as a commutator. Also, as e.g. $X_{i} \partial_{i}=\left[\partial_{1}, X_{1} X_{i} \partial_{i}\right]$ for $i \neq 1$ and $X_{1} \partial_{1}=\left[\partial_{1}, X_{1}^{(2)} \partial_{1}\right]$, every element of the Cartan subalgebra is a commutator of $\partial_{1}$, so $D_{H}$ is also a commutator.

To further investigate Brown's approach, we need to know about the automorphisms of $W(m, \underline{1})$. By Lemma 2.17, that every automorphism of $\mathcal{O}(m, \underline{1})$ induces an automorphism of $W(m, \underline{1})$. In fact, every automorphism of $W(m, \underline{1})$ is of that type.

Theorem 4.19 ([46, Theorem 7.3.1]). For every automorphism $\phi$ of $W(m, 1)$, there is an automorphism $\tilde{\phi}$ of $\mathcal{O}(m, \underline{1})$, such that

$$
\phi(D)=\tilde{\phi} \circ D \circ \tilde{\phi}^{-1}
$$

for all $D \in W(m, \underline{1})$, unless $p=3$ and $m=1$.

Theorem 4.20 ([46, Theorem 7.3.2]). Let $\phi$ be an automorphism of $\mathcal{O}(m, \underline{1})$, and $\bar{\phi}$ the induced automorphism of $W(m, \underline{1})$. Then the assignment $\phi \mapsto \bar{\phi}$ defines an isomorphism $\operatorname{Aut}(\mathcal{O}(m, \underline{1})) \simeq \operatorname{Aut}(W(m, \underline{1}))$.

Therefore, the problem of identifying the automorphisms of $W(m, \underline{1})$ boils down to identifying the automorphisms of $\mathcal{O}(m, \underline{1})$. As $\mathcal{O}(m, \underline{1})$ is a local ring with unique maximal ideal generated by $X_{1} \ldots, X_{m}$, this can be done easily.

Lemma 4.21. The homomorphism

$$
\begin{aligned}
\phi: \mathcal{O}(m, \underline{1}) & \rightarrow \mathcal{O}(m, \underline{1}), \\
X_{i} & \mapsto f_{i}
\end{aligned}
$$

with some polynomials $f_{i} \in \mathcal{O}(m, \underline{1})$ is an automorphism of $\mathcal{O}(m, \underline{1})$, if and only if

$$
f_{i}=\sum_{j=1}^{m} a_{i j} X_{j}+\text { terms of degree } \geqslant 2
$$

with $\operatorname{det}\left(a_{i j}\right) \neq 0$.

Proof. Assume first that $\phi$ is an automorphism. Denote the unique maximal ideal of $\mathcal{O}(m, \underline{1})$ by $\mathfrak{M}$. Then $\phi(\mathfrak{M}) \subseteq \mathfrak{M}$. Therefore, the degree-0-component of $\phi\left(X_{i}\right)$ must be trivial for every $i$.

Also, as $\phi\left(\mathfrak{M}^{2}\right) \subseteq \mathfrak{M}^{2}$, there is an induced isomorphism $\bar{\phi}$ of $\mathcal{O}(m, \underline{1}) / \mathfrak{M}^{2} \simeq F^{m}$ with

$$
\bar{\phi}\left(X_{i}+\mathfrak{M}^{2}\right)=\sum_{j=1}^{m} a_{i j} X_{j}+\mathfrak{M}^{2} .
$$


Therefore, $\operatorname{det}\left(a_{i j}\right) \neq 0$, as required.

For the converse, let $\phi$ be given by $\phi\left(X_{i}\right)=f_{i}$ with the $f_{i}$ as above. Let $\left(b_{i j}\right)=$ $\left(a_{i j}\right)^{-1}$. Define $\psi_{1}: \mathcal{O}(m, \underline{1}) \rightarrow \mathcal{O}(m, \underline{1})$ by $\psi_{1}\left(X_{i}\right)=\sum_{j=1}^{m} b_{i j} X_{j}$. Then $\left(\psi_{1} \circ\right.$ $\phi)\left(X_{i}\right)=X_{i}+r_{i, 1}$ with $r_{i, 1} \in \mathfrak{M}^{2}$. Continue by defining $\psi_{2}: X_{i} \mapsto X_{i}-r_{i, 1}$. Then $\left(\psi_{2} \circ \psi_{1} \circ \phi\right)\left(X_{i}\right)=\psi_{2}\left(X_{i}+r_{i, 1}\right)=X_{i}-r_{i, 1}+r_{i, 1}+r_{i, 2}=X_{i}+r_{i, 2}$ with $r_{i, 2} \in \mathfrak{M}^{3}$, and so on. As $\mathfrak{M}^{m(p-1)+1}=0$, we get $\psi_{m(p-1)} \circ \cdots \circ \psi_{1}=\phi^{-1}$, so $\phi$ is indeed an automorphism.

Let $\pi: \operatorname{Aut}(\mathcal{O}(m, \underline{1})) \rightarrow \mathrm{GL}_{m}(F)$ be given by $\pi(\phi)=\left(a_{i j}\right)$ with the notation of the Lemma above. Then $\pi$ is a surjective group homomorphism. Set $H=\operatorname{ker}(\pi)$. Then $H=\left\{\phi \in \operatorname{Aut}(\mathcal{O}(m, \underline{1})): \phi\left(X_{i}\right)=X_{i}+f_{i}, f_{i} \in \mathfrak{M}^{2}\right\}$, and $\operatorname{Aut}(\mathcal{O}(m, \underline{1})) / H \simeq$ $\mathrm{GL}_{m}(F)$. Thus we have a short exact sequence

$$
1 \longrightarrow H \longrightarrow \operatorname{Aut}(\mathcal{O}(m, \underline{1})) \stackrel{\pi}{\longrightarrow} \operatorname{GL}_{m}(F) \longrightarrow 1
$$

of groups. Clearly, this exact sequence splits, thus we obtain

$$
\operatorname{Aut}(\mathcal{O}(m, \underline{1})) \simeq H \rtimes \mathrm{GL}_{m}(F) .
$$

Now, after identifying all automorphisms of $W(m, \underline{1})$, one would hope that Lemma 4.3 holds for Witt algebras too. However, this is not the case as the following example shows.

Example 4.22. For every automorphism $\bar{\phi}$ of $W(1, \underline{1})$, we have $\bar{\phi}\left(X \partial_{X}\right) \notin E$.

Proof. Every automorphism of $W(1, \underline{1})$ is induced by an automorphism $\phi$ of $\mathcal{O}(1, \underline{1})$. These are given by $X \mapsto \sum_{i=1}^{p-1} a_{i} X^{i}$ with $a_{1} \neq 0$. Its inverse then is defined by $X \mapsto \sum_{j=1}^{p-1} b_{j} X^{j}$ with $b_{1}=a_{1}^{-1}$. Then

$$
\begin{aligned}
\bar{\phi}\left(X \partial_{X}\right)(X) & =\phi\left(X \partial_{X}\left(\sum_{j=1}^{p-1} b_{j} X^{j}\right)\right) \\
& =\sum_{j=1}^{p-1} \phi\left(j b_{j} X^{j}\right) \\
& =\sum_{j=1}^{p-1} j b_{j}\left(\sum_{i=1}^{p-1} a_{i} X^{i}\right)^{i}=X+f(X),
\end{aligned}
$$

where $f(X)$ involves only monomials of degree at least 2. Therefore, $\bar{\phi}\left(X \partial_{X}\right)=$ $X \partial_{X}+f(X) \partial_{X} \notin E$.

The above example shows that the exact copy of Brown's method does not work for Witt algebras. However, one could still hope that there are enough automorphisms, such that a given element can be mapped to an element which is already known to be a commutator, as it was done above for $W(2, \underline{1})$.

Another way of describing $W(m, \underline{1})$ is helpful here. Let $V=F^{m}$, and let $X_{1}, \ldots, X_{m}$ be a basis of $V$. Then $\operatorname{Sym}(V) \simeq F\left[X_{1}, \ldots, X_{m}\right]$, so $\mathcal{O}(m, \underline{1}) \simeq \operatorname{Sym}(V) / V^{p}=$ : 
$\operatorname{Sym}_{p}(V)$. The $\partial_{i}$ correspond to the dual basis of the given basis of $V$, and thus we have $W(m, \underline{1}) \simeq \operatorname{Sym}_{p}(V) \otimes V^{*}$.

Now the natural action of $\operatorname{GL}(V)$ on $V$ extends to an action on $\operatorname{Sym}_{p}(V)$. Also, $\mathrm{GL}(V)$ acts on $V^{*}$ by $A \cdot v^{*}=\left(A^{T}\right)^{-1} v^{*}$. Therefore, $\mathrm{GL}(V)$ acts on $W(m, \underline{1})$, so $\mathrm{GL}(V)$ embeds into the automorphism group of $W(m, \underline{1})$. Now, using the decomposition of $\operatorname{Sym}_{p}(V)$, we can write

$$
\operatorname{Sym}_{p}(V) \otimes V^{*}=\bigoplus_{i=0}^{p-1} \operatorname{Sym}^{i}(V) \otimes V^{*} .
$$

An element of $\operatorname{Sym}^{i}(V) \otimes V^{*}$ will be called homogeneous of degree $i-1$. As $\operatorname{Sym}^{0}(V) \simeq F$, and the action of $\operatorname{GL}(V)$ on $V^{*} \backslash\{0\}$ is transitive, for every $D \in$ $W(m, \underline{1})$ whose component in degree -1 is nonzero, there is $A \in \mathrm{GL}(V)$, such that the component of degree -1 of $A \cdot D$ is precisely $\partial_{1}$.

Thus assume that $D=\partial_{1}+D_{0}+D_{\geqslant 1}$, where $D_{0}$ is the degree-0-part of $D$, and $D_{\geqslant 1}$ the part of degree $\geqslant 1$. Let $D_{0}=\sum_{i, j} a_{i j} X_{j} \partial_{i}$. Let now $\phi \in \operatorname{Aut}(\mathcal{O}(m, \underline{1}))$ be the automorphism defined by

$$
\phi\left(X_{i}\right)=X_{i}+\sum_{j=1}^{m} a_{i j}^{\prime} X_{1} X_{j},
$$

where

$$
a_{i j}^{\prime}=\left\{\begin{array}{l}
a_{i j}, \text { if } j \neq 1, \\
a_{i 1} / 2, \text { if } j=1 .
\end{array}\right.
$$

Then one readily verifies that $\phi^{-1}\left(X_{i}\right)=X_{i}-\sum_{j} a_{i j}^{\prime} X_{1} X_{j}+g(\mathbf{X})$ with $\operatorname{deg}(g) \geqslant 3$. Therefore, for the induced automorphism $\bar{\phi}$, we have

$$
\begin{array}{r}
\bar{\phi}\left(\partial_{1}\right)\left(X_{i}\right)=\phi\left(\partial_{1}\left(X_{i}-\sum_{j} a_{i j}^{\prime} X_{1} X_{j}+g\right)\right) \\
=\phi\left(\delta_{i 1}-a_{11} X_{1}-\sum_{j \geqslant 2} a_{i j} X_{j}+g_{1}\right) \\
=\delta_{i 1}-a_{11}\left(X_{1}+\sum_{k} a_{1 k}^{\prime} X_{1} X_{k}\right)-\sum_{j \geqslant 2} a_{i j}\left(X_{j}+\sum_{k} a_{j k}^{\prime} X_{1} X_{k}\right)+\phi\left(g_{1}\right) .
\end{array}
$$

Therefore,

$$
\bar{\phi}\left(\partial_{1}\right)=\partial_{1}-\sum_{i} \sum_{j} a_{i j} X_{j} \partial_{i}+\text { terms of degree } \geqslant 1 .
$$

Therefore, $\bar{\phi}(D)$ has trivial degree-0-component, and is thus in particular in the complement of the Cartan subalgebra, and thus a commutator by Lemma 4.17. It remains to consider $D \in W(m, \underline{1})$ with trivial component in degree -1 . Now $\operatorname{Sym}^{1}(V) \otimes V \simeq \operatorname{End}(V)$, and one can calculate that the action of $\operatorname{GL}(V)$ on $\operatorname{Sym}^{1}(V) \otimes V$ is given by conjugation. This again shows that Lemma 4.3 fails for the Witt algebras, as the trace of a matrix is invariant under conjugation. Therefore, the degree-0-component of every conjugate of $X_{1} \partial_{1}$ has "trace" 1 , and 
thus cannot be an element of $E$. However, as $F$ is algebraically closed, we can assume $D_{0}$ to be in Jordan normal form, i.e. of the form

$$
D_{0}=\sum_{i=1}^{m} \lambda_{i} X_{i} \partial_{i}+\sum_{i=1}^{m-1} a_{i+1, i} X_{i+1} \partial_{i}
$$

with $\lambda_{i} \in F, a_{i, i+1} \in\{0,1\}$ and $\lambda_{i}=\lambda_{i+1}$ if $a_{i+1, i}=1$.

Now, as we cannot get rid of the degree-0-component of an element with $D_{-1}=0$, we would like to apply an automorphism to such an element to map it to another element which is known to be a commutator. One could for example hope to find an automorphism $\phi \in \operatorname{Aut}(W(m, \underline{1}))$, such that $\phi(D)=\sum_{i} f_{i} \partial_{i}$, where every $f_{i}$ can be integrated with respect to $X_{1}$. Then obviously $\phi(D)=\left[\partial_{1}, D^{\prime}\right]$ for some $D^{\prime} \in W(m, \underline{1})$. The way one would try to construct such an automorphism would be to delete the components in each degree step by step, starting in degree 1 by applying an automorphism induced by an automorphism of $\mathcal{O}(m, \underline{1})$ of the type $X_{i} \mapsto X_{i}+f_{i}$ with $f_{i}$ homogeneous of degree 2 . However, this might not always be possible, as the following calculation for $m=2$ shows.

Assume that, after applying an automorphism given by an element of GL $(V)$, we have a diagonal $D_{0}$-component, i.e. $D_{0}=\lambda X \partial_{X}+\mu Y \partial_{Y}$ for some $\lambda, \mu \in$ $F$, and arbitrary degree-1-component. We want to find an automorphism $\phi \in$ $\operatorname{Aut}(\mathcal{O}(m, \underline{1}))$ given by

$$
\begin{aligned}
& \phi(X)=X+f_{1} X^{2}+f_{2} X Y+f_{3} Y^{2} \\
& \phi(Y)=Y+g_{1} X^{2}+g_{2} X Y+g_{3} Y^{2}
\end{aligned}
$$

with some $f_{1}, f_{2}, f_{3}, g_{1}, g_{2}, g_{3} \in F$. Then one can compute that

$\bar{\phi}\left(X \partial_{X}\right)=X \partial_{X}+\left(-f_{1} X^{2}+f_{3} Y^{2}\right) \partial_{X}+\left(-2 g_{1} X^{2}-g_{2} X Y\right) \partial_{Y}+$ terms of higher degree and analogously

$\bar{\phi}\left(Y \partial_{Y}\right)=Y \partial_{Y}+\left(g_{1} X^{2}-g_{3} Y^{2}\right) \partial_{Y}+\left(-f_{2} X Y-2 f_{3} Y^{2}\right) \partial_{X}+$ terms of higher degree.

Therefore, we get

$$
\begin{aligned}
\bar{\phi}\left(\lambda X \partial_{X}+\mu Y \partial_{Y}\right) & =\left(-\lambda f_{1} X^{2}-\mu f_{2} X Y+(\lambda-2 \mu) f_{3} Y^{2}\right) \partial_{X} \\
& +\left((-2 \lambda+\mu) g_{1} X^{2}-\lambda g_{2} X Y-\mu g_{3} Y^{2}\right) \partial_{Y}
\end{aligned}
$$

Thus, if $\lambda=0, \mu=0, \lambda-2 \mu=0$ etc., and the corresponding coefficient in $D_{1}$ is nonzero, there is no chance of finding $\phi$, such that the degree-1-component is deleted after applying $\bar{\phi}$.

\section{7 $\quad$ Summarizing the Results}

Let us now summarize what we achieved about proving the Ore conjecture for the Witt algebras $W(m, \underline{n})$.

Firstly, we were able to prove the Ore conjecture for the Witt algebras $W(1, \underline{n})$ 
in one variable for every $n \geqslant 1$, irrespective of the base field. The proof worked in a purely combinatorial way, using only the standard basis and its structure constants. The advantage of this proof is that it does not require a lot of background knowledge, and also it gives an explicit construction of how to write a given element as a commutator. The downside however is, that the proof is hard to generalize, as the calculations become too long and too many cases have to be distinguished.

Thus, we started a different approach, using algebraic geometry and intersection theory. As intersection theory often requires the field to be algebraically closed, we added that assumption to our base field. We could then reprove quite easily and in a much shorter way that every element of $W(1, \underline{1})$ is a commutator, though not giving an explicit construction.

When trying to generalize this approach to the Witt algebra $W(2, \underline{1})$, the first problem occured. Namely, the element corresponding to the intersection point of the given varieties may not be regular. However, we were able to use an appropriate automorphism of $W(2, \underline{1})$ to "push the element into the safe zone", and the proof of the Ore conjecture for $W(2, \underline{1})$ could be completed. However, for $m \geqslant 3$, the set of "bad" points becomes bigger and harder to control. Therefore, the proof could not be eztended to a proof for the Ore conjecture for $W(3, \underline{1})$.

Therefore, we tried to adapt the proof for the Ore conjecture for the classical Lie algebras to $W(m, \underline{1})$, making more use of the automorphism group of $W(m, \underline{1})$. While one of the two main Lemmas of Brown does have an equivalent for the Witt algebras, the other Lemma is not true there. However, the first Lemma is sufficient to prove that every element of $W(m, \underline{1})$ can be written as a sum of at most two commutators. Now, as $W(m, \underline{1})$ is a graded Lie algebra, the srategy was to apply automorphisms to control the element in each degree, until the element is transformed into an element already known to be a commutator. This, however, turned out to be not as smooth as one would have hoped, though again a large class of elements of $W(m, \underline{1})$ could be proved to be a commutator.

Putting together the facts we obtained, it is hard to imagine that there is an element of $W(m, \underline{1})$, which is not a commutator. We were often quite close to achieving the result, but the final cases which had to be analyzed turned out to become hard to prove and hard to generalize. We do however think, that it is possible to obtain the result in the near future.

Once this is done, one can of course ask the same question for $W(m, \underline{n})$ and the other finite dimensional simple Lie algebras of Cartan type, namely the hamiltonian algebras, the special algebras and the contact algebras. 


\section{Bibliography}

[1] A. A. Albert and Benjamin Muckenhoupt. On matrices of trace zeros. Michigan Math. J., 4:1-3, 1957.

[2] Laurent Bartholdi and Roman Mikhailov. Homotopy groups of spheres and dimension quotients. arXiv e-prints, page arXiv:1805.10894, May 2018.

[3] Laurent Bartholdi and Inder Bir S. Passi. Lie dimension subrings. Internat. J. Algebra Comput., 25(8):1301-1325, 2015.

[4] George M. Bergman and Warren Dicks. On universal derivations. J. Algebra, 36(2):193-211, 1975.

[5] Oliver Bonten. Über Kommutatoren in endlichen einfachen Gruppen. Aachen: RWTH, 1993.

[6] Gordon Brown. On commutators in a simple Lie algebra. Proc. Amer. Math. Soc., 14:763-767, 1963.

[7] P. Cartier. Remarques sur le théorème de Birkhoff-Witt. Ann. Scuola Norm. Sup. Pisa (3), 12:1-4, 1958.

[8] G. Cliff and B. Hartley. Sjogren's theorem on dimension subgroups. J. Pure Appl. Algebra, 47(3):231-242, 1987.

[9] P. M. Cohn. Generalization of a theorem of Magnus. Proc. London Math. Soc. (3), 2:297-310, 1952.

[10] P. M. Cohn. A remark on the Birkhoff-Witt theorem. J. London Math. Soc., 38:197-203, 1963.

[11] Erich W. Ellers and Nikolai Gordeev. On the conjectures of J. Thompson and O. Ore. Trans. Amer. Math. Soc., 350(9):3657-3671, 1998.

[12] Adriano M. Garsia. Combinatorics of the free Lie algebra and the symmetric group. In Analysis, et cetera, pages 309-382. Academic Press, Boston, MA, 1990.

[13] R. Gow. Commutators in the symplectic group. Arch. Math. (Basel), 50(3):204-209, 1988.

[14] Luzius Grünenfelder. Lower central series, augmentation quotients and homology of groups. Comment. Math. Helv., 55(2):159-177, 1980.

[15] N. D. Gupta, A. W. Hales, and I. B. S. Passi. Dimension subgroups of metabelian groups. J. Reine Angew. Math., 346:194-198, 1984.

[16] Narain Gupta. On the dimension subgroups of metabelian groups. J. Pure Appl. Algebra, 24(1):1-6, 1982. 
[17] Narain Gupta. Free group rings, volume 66 of Contemporary Mathematics. American Mathematical Society, Providence, RI, 1987.

[18] Narain Gupta. Sjogren's theorem for dimension subgroups - the metabelian case. In Combinatorial group theory and topology (Alta, Utah, 1984), volume 111 of Ann. of Math. Stud., pages 197-211. Princeton Univ. Press, Princeton, NJ, 1987.

[19] Narain Gupta. The dimension subgroup conjecture. Bull. London Math. Soc., 22(5):453-456, 1990.

[20] Narain Gupta. A solution of the dimension subgroup problem. J. Algebra, 138(2):479-490, 1991.

[21] Robin Hartshorne. Algebraic geometry. Springer-Verlag, New YorkHeidelberg, 1977. Graduate Texts in Mathematics, No. 52.

[22] Nathan Jacobson. Lie algebras. Interscience Tracts in Pure and Applied Mathematics, No. 10. Interscience Publishers (a division of John Wiley \& Sons), New York-London, 1962.

[23] S. A. Jennings. The group ring of a class of infinite nilpotent groups. Canad. J. Math., 7:169-187, 1955.

[24] Frans Keune. Derived functors and algebraic $K$-theory. In Algebraic $K$ theory, I: Higher K-theories (Proc. Conf. Seattle Res. Center, Battelle Memorial Inst., 1972), pages 166-176. Lecture Notes in Math., Vol. 341, 1973.

[25] Serge Lang. Algebra, volume 211 of Graduate Texts in Mathematics. SpringerVerlag, New York, third edition, 2002.

[26] Michel Lazard. Sur les groupes nilpotents et les anneaux de Lie. Ann. Sci. Ecole Norm. Sup. (3), 71:101-190, 1954.

[27] Martin W. Liebeck, E. A. O'Brien, Aner Shalev, and Pham Huu Tiep. The Ore conjecture. J. Eur. Math. Soc. (JEMS), 12(4):939-1008, 2010.

[28] Saunders Mac Lane. Homology. Die Grundlehren der mathematischen Wissenschaften, Bd. 114. Academic Press, Inc., Publishers, New York; SpringerVerlag, Berlin-Göttingen-Heidelberg, 1963.

[29] Wilhelm Magnus. Über Beziehungen zwischen höheren Kommutatoren. $J$. Reine Angew. Math., 177:105-115, 1937.

[30] Wilhelm Magnus. Über Gruppen und zugeordnete Liesche Ringe. J. Reine Angew. Math., 182:142-149, 1940.

[31] Wilhelm Magnus, Abraham Karrass, and Donald Solitar. Combinatorial group theory. Dover Publications, Inc., Mineola, NY, second edition, 2004. Presentations of groups in terms of generators and relations. 
[32] J. Peter May. Simplicial objects in algebraic topology. Chicago Lectures in Mathematics. University of Chicago Press, Chicago, IL, 1992. Reprint of the 1967 original.

[33] Roman Mikhailov and Inder Bir Singh Passi. Lower central and dimension series of groups, volume 1952 of Lecture Notes in Mathematics. SpringerVerlag, Berlin, 2009.

[34] Oystein Ore. Some remarks on commutators. Proc. Amer. Math. Soc., 2:307314, 1951.

[35] Inder Bir S. Passi. Group rings and their augmentation ideals, volume 715 of Lecture Notes in Mathematics. Springer, Berlin, 1979.

[36] Inder Bir S. Passi and Thomas Sicking. Dimension quotients of metabelian Lie rings. Internat. J. Algebra Comput., 27(2):251-258, 2017.

[37] Christophe Reutenauer. Free Lie algebras, volume 7 of London Mathematical Society Monographs. New Series. The Clarendon Press, Oxford University Press, New York, 1993. Oxford Science Publications.

[38] David M. Riley. The dimension subalgebra problem for enveloping algebras of Lie superalgebras. Proc. Amer. Math. Soc., 123(10):2975-2980, 1995.

[39] E. Rips. On the fourth integer dimension subgroup. Israel J. Math., 12:342346, 1972.

[40] Robert Sandling and Ken-Ichi Tahara. Augmentation quotients of group rings and symmetric powers. Math. Proc. Cambridge Philos. Soc., 85(2):247-252, 1979.

[41] Hans-Georg Schumann. Über Moduln und Gruppenbilder. Math. Ann., 114(1):385-413, 1937.

[42] G. B. Seligman. Modular Lie algebras. Ergebnisse der Mathematik und ihrer Grenzgebiete, Band 40. Springer-Verlag New York, Inc., New York, 1967.

[43] Jean-Pierre Serre. Lie algebras and Lie groups, volume 1500 of Lecture Notes in Mathematics. Springer-Verlag, Berlin, second edition, 1992. 1964 lectures given at Harvard University.

[44] Kenjiro Shoda. Einige Sätze über Matrizen. Jpn. J. Math., 13(3):361-365, 1937.

[45] J. A. Sjogren. Dimension and lower central subgroups. J. Pure Appl. Algebra, 14(2):175-194, 1979.

[46] Helmut Strade. Simple Lie algebras over fields of positive characteristic. I, volume 38 of De Gruyter Expositions in Mathematics. Walter de Gruyter \& Co., Berlin, 2004. Structure theory. 
[47] Helmut Strade and Rolf Farnsteiner. Modular Lie algebras and their representations, volume 116 of Monographs and Textbooks in Pure and Applied Mathematics. Marcel Dekker, Inc., New York, 1988.

[48] Ken-Ichi Tahara. The augmentation quotients of group rings and the fifth dimension subgroups. J. Algebra, 71(1):141-173, 1981.

[49] R. C. Thompson. Commutators in the special and general linear groups. Trans. Amer. Math. Soc., 101:16-33, 1961.

[50] R. C. Thompson. Commutators of matrices with coefficients from the field of two elements. Duke Math. J., 29:367-373, 1962.

[51] R. C. Thompson. On matrix commutators. Portugal. Math., 21:143-153, 1962.

[52] Ernst Witt. Treue Darstellung Liescher Ringe. J. Reine Angew. Math., 177:152-160, 1937. 